JOURNAL OF THE

AMERICAN MATHEMATICAL SOCIETY

Volume 9, Number 4, October 1996

\title{
MODEL COMPLETENESS RESULTS FOR EXPANSIONS OF THE ORDERED FIELD OF REAL NUMBERS BY RESTRICTED PFAFFIAN FUNCTIONS AND THE EXPONENTIAL FUNCTION
}

\author{
A. J. WILKIE
}

\section{INTRODUCTION}

Recall that a subset of $\mathbb{R}^{n}$ is called semi-algebraic if it can be represented as a (finite) boolean combination of sets of the form $\left\{\vec{\alpha} \in \mathbb{R}^{n}: p(\vec{\alpha})=0\right\}$, $\left\{\vec{\alpha} \in \mathbb{R}^{n}: q(\vec{\alpha})>0\right\}$ where $p(\vec{x}), q(\vec{x})$ are $n$-variable polynomials with real coefficients. A map from $\mathbb{R}^{n}$ to $\mathbb{R}^{m}$ is called semi-algebraic if its graph, considered as a subset of $\mathbb{R}^{n+m}$, is so. The geometry of such sets and maps ("semi-algebraic geometry") is now a widely studied and flourishing subject that owes much to the foundational work in the 1930s of the logician Alfred Tarski. He proved ([11]) that the image of a semi-algebraic set under a semi-algebraic map is semi-algebraic. (A familiar simple instance: the image of $\left\{\langle a, b, c, x\rangle \in \mathbb{R}^{4}: a \neq 0\right.$ and $\left.a x^{2}+b x+c=0\right\}$ under the projection map $\mathbb{R}^{3} \times \mathbb{R} \rightarrow \mathbb{R}^{3}$ is $\left\{\langle a, b, c\rangle \in \mathbb{R}^{3}: a \neq 0\right.$ and $\left.b^{2}-4 a c \geq 0\right\}$.) Tarski's result implies that the class of semi-algebraic sets is closed under firstorder logical definability (where, as well as boolean operations, the quantifiers " $\exists x \in \mathbb{R} \ldots$. and " $\forall x \in \mathbb{R} \ldots$. are allowed) and for this reason it is known to logicians as "quantifier elimination for the ordered ring structure on $\mathbb{R}$ ". Immediate consequences are the facts that the closure, interior and boundary of a semialgebraic set are semi-algebraic. It is also the basis for many inductive arguments in semi-algebraic geometry where a desired property of a given semi-algebraic set is inferred from the same property of projections of the set into lower dimensions. For example, the fact (due to Hironaka) that any bounded semi-algebraic set can be triangulated is proved this way.

In the 1960s the analytic geometer Eojasiewicz extended the above theory to the analytic context ([8]). The definition of a semi-analytic subset of $\mathbb{R}^{n}$ is the same as above except that for the basic sets the $p(\vec{x})$ 's and $q(\vec{x})$ 's are allowed to be analytic functions and we only insist that the boolean representations work locally around each point of $\mathbb{R}^{n}$ (allowing different representations around different points). It is also necessary to restrict the maps to be proper (with semi-analytic graph). With this restriction it is true that the image of a semi-analytic set, known as a sub-analytic set, is semi-analytic provided that the target space is either $\mathbb{R}$ or $\mathbb{R}^{2}$. Counterexamples have been known since the beginning of this century for maps to $\mathbb{R}^{m}$ for $m \geq 3$. (They are due to Osgood, see [8].) However, the situation was clarified in 1968 by Gabrielov ([5]) who showed that the class of sub-analytic sets

Received by the editors July 28, 1992 and, in revised form, June 29, 1995.

1991 Mathematics Subject Classification. Primary 03C10.

(C)1996 American Mathematical Society 
is closed under taking complements. Gabrielov's theorem can be reformulated in terms of logical definability as follows.

For each $m$ and each analytic function $f: U \rightarrow \mathbb{R}$, where $U$ is some open neighbourhood of the closed box $[0,1]^{m}$ in $\mathbb{R}^{m}$, let $\tilde{f}: \mathbb{R}^{m} \rightarrow \mathbb{R}$ be defined by

$$
\tilde{f}(\vec{x})= \begin{cases}f(\vec{x}) & \text { if } \vec{x} \in[0,1]^{m} \\ 0 & \text { if } \vec{x} \in \mathbb{R}^{m} \backslash[0,1]^{m}\end{cases}
$$

Let $L_{a n}$ denote the language extending that of ordered rings obtained by adding a function symbol for each such function $\tilde{f}$. Then Gabrielov's theorem is readily seen to be equivalent to the assertion that for each $n$, every subset of $\mathbb{R}^{n}$ which can be defined by some logical formula of the language $L_{a n}$ can, in fact, be defined by an existential formula of $L_{a n}$, that is, one of the form $\exists y_{1}, \ldots, y_{r} \phi\left(x_{1}, \ldots, x_{n}, y_{1}, \ldots, y_{r}\right)$ where the $L_{a n}$-formula $\phi$ contains no occurrences of quantifiers. (Further, the class of all such subsets which are bounded is exactly the class of bounded sub-analytic subsets of $\mathbb{R}^{n}$. At first sight it might seem that the former class is richer because the projection map implicit in the existential quantification is not restricted to a compact set. The fact that we do not obtain non-sub-analytic sets is due, of course, to our original truncation of the analytic functions.) In this form Gabrielov's theorem was given a fairly straightforward treatment, based on the Weierstrass preparation theorem and Tarski's elimination theory, by Denef and van den Dries ([3]).

Thus, although we do not have full quantifier elimination for this local analytic structure (together with the ordered ring structure) on $\mathbb{R}$, we do have elimination down to existential formulas. Such structures are called model complete, a term introduced by Abraham Robinson. Actually, whether or not a structure is model complete only depends on the theory of the structure, that is on the set of all sentences of its language (a sentence is a formula without free variables) that are true in the structure. More generally, if $T$ is a consistent set of sentences of some language $L$, then $T$ is called model complete if for every formula $\psi(\vec{x})$ of $L$ there is an existential formula $\theta(\vec{x})$ of $L$ such that the sentence $\forall \vec{x}(\psi(x) \leftrightarrow \theta(\vec{x}))$ is a formal consequence of $T$. Further, if $\theta(\vec{x})$ can always be chosen to contain no occurrences of quantifiers at all, then $T$ is said to admit elimination of quantifiers.

To summarize the above discussion, then, let $\overline{\mathbb{R}}=\langle\mathbb{R} ;+, \cdot,-, 0,1,<\rangle$ and $\mathbb{R}_{a n}=$ $\langle\overline{\mathbb{R}} ; \mathcal{F}\rangle$ where $\mathcal{F}$ consists of all functions of the form $\tilde{f}$ as described above. Let $\bar{T}$ and $T_{a n}$ denote the theories of these structures respectively. Then $\bar{T}$ admits elimination of quantifiers (Tarski) and $T_{a n}$ is model complete (Gabrielov) but does not admit elimination of quantifiers (Osgood).

My aim in this paper is to give two variations of Gabrielov's theme. The first is in response to the following natural question: when can the analytic functions needed to describe the complement of a given sub-analytic set be chosen from the ring generated by functions used to describe the given set? Or, in model theoretic terms, for which subsets $\mathcal{G}$ of $\mathcal{F}$ is (the theory of) the structure $\langle\mathbb{R} ; \mathcal{G}\rangle$ model complete? I shall show that this is the case when $\mathcal{G}$ is a Pfaffian chain of functions. Let me make this more precise.

Firstly, fix $m, l \in \mathbb{N}, m, l \geq 1$, and an open set $U \subseteq \mathbb{R}^{m}$ such that the closed box $[0,1]^{m}$ is contained in $U$. Let $G_{1}, \ldots, G_{l}: U \rightarrow \mathbb{R}$ be analytic functions and suppose that there exist polynomials $p_{i, j} \in \mathbb{R}\left[z_{1}, \ldots, z_{m+i}\right]$ (for $i=1, \ldots, l, j=1, \ldots, m$ ) 
such that

$$
\frac{\partial G_{i}}{\partial x_{j}}(\vec{x})=p_{i, j}\left(\vec{x}, G_{1}(\vec{x}), \ldots, G_{i}(\vec{x})\right) \quad(\text { for all } \vec{x} \in U) .
$$

The sequence $G_{1}, \ldots, G_{l}$ is called a Pfaffian chain on $U$. Let $F_{1}, \ldots, F_{l}$ be the corresponding truncations. That is,

$$
F_{i}(\vec{x})= \begin{cases}G_{i}(\vec{x}) & \text { if } \vec{x} \in[0,1]^{m}, \\ 0 & \text { if } \vec{x} \in \mathbb{R}^{m} \backslash[0,1]^{m} .\end{cases}
$$

Now let $C$ be any subset of $\mathbb{R}$ such that each coefficient of each $p_{i, j}$ is the value of some term (without free variables) in the structure $\left\langle\overline{\mathbb{R}} ; F_{1}, \ldots, F_{l} ; r\right\rangle_{r \in C}$. (For example, we could take $C$ to be the set of all such coefficients.) I denote this structure by $\widetilde{\mathbb{R}}$ and its language and theory by $\widetilde{L}, \widetilde{T}$, respectively.

Obviously $\widetilde{L}$ is a sublanguage of $L_{a n}$ and every subset of $\mathbb{R}^{n}$ that can be defined by a formula of $\widetilde{L}$ can be defined by a formula, and hence an existential formula, of $L_{a n}$. I shall prove the following.

First Main Theorem. Every subset of $\mathbb{R}^{n}$ (for any $n$ ) that can be defined by some formula of $\widetilde{L}$ can be defined by an existential formula of $\widetilde{L}$. That is, $\widetilde{T}$ is model complete.

Examples. (A) Take $m=l=1, U=\mathbb{R}, G_{1}\left(x_{1}\right)=\exp \left(x_{1}\right), p_{1,1}\left(z_{1}, z_{2}\right)=z_{2}$ and $C=\emptyset$. Then the theorem tells us that the theory of the structure $\langle\overline{\mathbb{R}}$; $\exp \lceil[0,1]\rangle$ is model complete. Of course the convention (2) dictates that $\exp \lceil[0,1]$ is defined to be 0 outside $[0,1]$. If one prefers to have only functions that are analytic throughout $\mathbb{R}\left(\right.$ or $\mathbb{R}^{m}$ ) in the basic language, then one can always invoke the following cosmetic trick. Define $e: \mathbb{R} \rightarrow \mathbb{R}, x \rightarrow \exp \left(\left(1+x^{2}\right)^{-1}\right)$. Then the structures $\langle\overline{\mathbb{R}} ; \exp \lceil[0,1]\rangle$ and $\langle\overline{\mathbb{R}} ; e\rangle$ are essentially the same, i.e. they have the same definable sets and, more to the point, the same existentially definable sets. It follows that the theory of the structure $\langle\overline{\mathbb{R}} ; e\rangle$ is model complete.

(B) Sometimes the cosmetic trick comes for free. Take $m=1, l=2, U=\mathbb{R}$, $G_{1}\left(x_{1}\right)=\left(1+x_{1}^{2}\right)^{-1}, G_{2}\left(x_{1}\right)=\tan ^{-1}\left(x_{1}\right), p_{1,1}\left(z_{1}, z_{2}\right)=2 z_{1} z_{2}^{2}, p_{2,1}\left(z_{1}, z_{2}, z_{3}\right)=z_{2}$ and $C=\emptyset$. Since the graph of the function $G_{1}$ (or rather $F_{1}$ ) is already definable in $\overline{\mathbb{R}}$ by a quantifier-free formula, the theorem implies that the theory of the structure $\left\langle\mathbb{R} ; \tan ^{-1}\lceil[0,1]\rangle\right.$ is model complete. But in this case we have functional equations at $\pm \infty$, namely

$$
\tan ^{-1}\left(\frac{1}{x}\right)=\frac{\pi}{2}-\tan ^{-1}(x) \text { for } x>0
$$

and

$$
\tan ^{-1}\left(\frac{1}{x}\right)=-\frac{\pi}{2}-\tan ^{-1}(x) \quad \text { for } x<0
$$

which, together with the equations

$$
\tan ^{-1}(-x)=-\tan ^{-1}(x) \text { and } \frac{\pi}{2}=2 \tan ^{-1}(1),
$$

clearly imply that the theory of the structure $\left\langle\overline{\mathbb{R}} ; \tan ^{-1}\right\rangle\left(\right.$ with $\tan ^{-1}$ unrestricted) is model complete. 
(C) As far as I know the first result along these lines was obtained by van den Dries ([15]) who showed that the theory of the structure

$$
\left\langle\overline{\mathbb{R}} ; \sin \uparrow[0,1], \exp \lceil[0,1] ; r\rangle_{r \in \mathbb{R}}\right.
$$

is model complete. This also follows from the first main theorem by combining the chains used in (A) and (B) and then invoking elementary trigonometric identities. I leave the details to the reader. (The reason van den Dries actually needs the sine function is that his proof uses complex power series methods and the required model completeness is then deduced, by "taking real parts", from a corresponding result for complex exponentiation restricted to the unit disc. The key point in this approach is that the complex analytic functions cropping up as coefficients in the Weierstrass Preparation Theorem can be existentially defined from the initial data (and, possibly, extra parameters - hence the choice $C=\mathbb{R}$ here) - a fact that, interestingly, seems to be unknown in the case of the Preparation Theorem for real analytic functions.)

Whether one uses analytic or model-theoretic terminology and methods, the proofs of all the above results work because one only ever has to deal with analytic functions restricted to compact subsets of their natural domains or, equivalently (via the cosmetic trick), with total analytic functions that are also analytic at infinity. The second result of this paper removes this restriction in one particular case.

Second Main Theorem. The theory of the structure $\langle\overline{\mathbb{R}} ; \exp \rangle$, where exp is the usual exponential function $x \mapsto e^{x}$ with domain $\mathbb{R}$, is model complete.

Thus, if we define a subset of $\mathbb{R}^{n}$ to be semi-EA ("semi-exponential-algebraic") if it can be represented as a boolean combination of sets of the form $\left\{\vec{\alpha} \in \mathbb{R}^{n}\right.$ : $p(\vec{\alpha})=0\},\left\{\vec{\alpha} \in \mathbb{R}^{n}: q(\vec{\alpha})>0\right\}$, where the $p(\vec{x})$ 's and $q(\vec{x})$ 's are exponential polynomials (i.e. polynomials in $x_{1}, \ldots, x_{n}, e^{x_{1}}, \ldots, e^{x_{n}}$ with real (or, more generally (!), integer) coefficients), and a map from $\mathbb{R}^{n}$ to $\mathbb{R}^{m}$ to be semi-EA if its graph is so (and we do not demand that the map be proper) and, finally, a set to be $s u b-E A$ if it is the image of a semi-EA set under a semi-EA map, then the theorem is equivalent to the assertion that the complement of a sub-EA set is a sub-EA set. This, as for the semi-algebraic case, implies that the class of sub-EA sets is also closed under taking closures, interiors and boundaries.

It is difficult to see how conventional analytic or differential geometric methods could be used to establish this result because of the essential singularity of the exponential function at infinity. The proof given here uses model-theoretic methods to analyse large zeros of systems of exponential-algebraic equations.

Before giving a plan of the paper I should make a few remarks concerning effectivity. For it was Tarski's main purpose in his paper to show not only that every formula of $\bar{L}$ is equivalent (modulo $\bar{T}$ ) to a quantifier-free formula, but also that the latter could be found effectively from the former. From this he deduced that $\bar{T}$ is a decidable theory, i.e. there exists an (explicitly given) algorithm to decide whether or not an arbitrary sentence of $\bar{L}$ is true in $\overline{\mathbb{R}}$ (hence the title of the paper). Tarski asked whether this holds for $\langle\overline{\mathbb{R}}$; $\exp \rangle$ and while this question was the motivation for the work in this paper, I feel it would have obscured the arguments here had I paid constant attention to effectivity considerations. Such problems will be discussed in a forthcoming paper of A. J. Macintyre and the author, where they will be shown 
to be intimately linked with the conjecture of Schanuel in transcendental number theory.

For an introduction to the general notion of model completeness I refer the reader to [1]. Several equivalent formulations are mentioned there (and, in fact, the definition I have given is not Robinson's original one but one of these equivalents) including the following: if $T$ is a consistent set of sentences in a language $L$, then $T$ is model complete if and only if whenever $\mathfrak{A}, \mathfrak{B}$ are models of $T$ with $\mathfrak{A} \subseteq \mathfrak{B}$ (i.e. $\mathfrak{A}$ is an $L$-substructure of $\mathfrak{B}$ ), then $\mathfrak{G}$ is existentially closed in $\mathfrak{B}$. For the theories involved in the two main theorems above (or, indeed, for the theory of any structure expanding $\overline{\mathbb{R}}$ by functions and constants) establishing the latter is equivalent to showing that any finite set of equations (involving the basic functions of the given language) with parameters from $\mathfrak{A}$ is solvable in $\mathfrak{A}$ provided that it is solvable in $\mathfrak{B}$. This is how I shall go about proving the theorems.

The next section clarifies this approach and organizes the equations that we need to solve into manageable form. After summarizing known finiteness theorems for the solutions of such equations in section 3 I develop, in sections 4 and 5 , a theory of Noetherian rings of differentiable germs that works for arbitrary (possibly nonstandard) models of suitable theories. (As an application we give a proof (see 5.3) of the theorem of Khovanskii stating that Pfaffian varieties have only finitely many connected components.) Sections 6 and 7 are rather tedious. This is because I need to develop some very elementary, but global, existence theorems from the differential calculus that apply to, as above, arbitrary models of the theories under consideration and this can only be accomplished, as far as I can see, by exhibiting explicit definitions. Many algebraic manipulations (especially of Jacobian matrices) are involved here, the details of which may be safely skipped without loss of understanding of the main arguments.

For all the results of sections 2 to 7 (apart from 3.4 and 3.5) it is irrelevant whether or not the basic functions are restricted to the closed unit box and so they apply to the situations of both main theorems. I have, however, concentrated on those structures to which the first theorem applies because the truncation actually introduces extra difficulties (of a rather superficial nature). Hereafter the proofs diverge because we need to confront the problem, briefly referred to above, of large solutions of the equations under consideration. This is done in section 8 for the first theorem, thus completing its proof. Sections 9 to 11 are devoted to the completion of the proof of the second theorem. These may be read independently as I restate the necessary results from earlier sections.

Tarski's problem on the real exponential function has been the focus of papers by many authors. Apart from those mentioned above I refer the reader to the pioneering work of Dahn ([2]) and Wolter ([17]). For the crucial inequalities needed in the proof of the model completeness of the structure $\langle\overline{\mathbb{R}}$; exp $\rangle$ can be viewed as a generalization to many variables of the Dahn bounding theorem ([2]).

\section{TOWARDS THE PROOF OF THE FIRST MAIN THEOREM}

The symbols $\widetilde{K}, \tilde{k}$ will denote $\widetilde{L}$-structures with domains $K, k$ respectively, although I shall sometimes use $K, k$ to denote the underlying fields or ordered fields. If $\tilde{k} \subseteq \widetilde{K}$, then $\widetilde{L}_{k}$ (respectively $\bar{L}_{k}$ ) denotes the expansion of $\widetilde{L}$ (respectively $\bar{L}$ ) obtained by adding a new constant symbol for each element of $k$. The corresponding $\widetilde{L}_{k}$-expansion of $\widetilde{K}$ will be denoted simply $\widetilde{K}^{+}$when it is clear which $k$ is 
intended. I shall also adopt the usual practice of not distinguishing notationally between non-logical symbols of a language and their interpretation in a structure under consideration. In particular, for $i=1, \ldots, l$, the symbol $F_{i}$, which was introduced in section 1 as a particular function from $\mathbb{R}^{m}$ to $\mathbb{R}$, will also denote the corresponding function symbol of $\widetilde{L}$ as well as the function from $K^{m}$ to $K$ that the function symbol is interpreted as in a given $\widetilde{L}$-structure $\widetilde{K}$.

Now for $1 \leq i \leq l, 1 \leq j \leq m$ and $n \geq 1,(1)$ and (2) imply

$$
F_{i} \text { is } n \text {-times differentiable on the open box }(0,1)^{m} \text {, }
$$

and

$$
\forall \vec{x} \in(0,1)^{m}, \quad \frac{\partial F_{i}}{\partial x_{j}}=p_{i, j}\left(\vec{x}, F_{1}(\vec{x}), \ldots, F_{i}(\vec{x})\right) .
$$

Clearly (3) and (4) can be expressed by sentences of $\widetilde{L}$ (note the property of the set $C$ ) and these sentences are therefore in $\widetilde{T}$. But there is no obvious way to express the fact that $F_{i}$ (restricted to $(0,1)^{m}$ ) has an analytic continuation (namely $G_{i}$ ) to an open set containing the closed box $[0,1]^{m}$ (namely $U$ ) such that (1) holds. However, as the remarks following example (C) above were intended to suggest, we must use this fact and, indeed, there are several consequences of it that are first-order expressible and I need to mention one such here.

Let $S \subseteq\{1, \ldots, m\}$ and suppose $a_{j} \in\{0,1\}$ for $j \in S$. Define the functions $F_{i}^{*}: \mathbb{R}^{m} \rightarrow \mathbb{R}$ by $F_{i}^{*}\left(x_{1}, \ldots, x_{m}\right)=F_{i}\left(x_{1}^{\prime}, \ldots, x_{m}^{\prime}\right)$ where

$$
x_{j}^{\prime}= \begin{cases}x_{j} & \text { if } j \notin S \\ a_{j} & \text { if } j \in S .\end{cases}
$$

Let

$$
J_{j}= \begin{cases}(0,1) & \text { if } j \notin S \\ \mathbb{R} & \text { if } j \in S .\end{cases}
$$

Then (1) and (2) imply (with $i, j, n$ as above)

$$
F_{i}^{*} \text { is } n \text {-times differentiable on the open set } \prod_{j=1}^{m} J_{j} \text {, }
$$

and

(6) $\forall \vec{x} \in \prod_{j=1}^{m} J_{j}, \quad \frac{\partial F_{i}^{*}}{\partial x_{j}}(\vec{x})=\left\{\begin{array}{l}P_{i, j}\left(x_{1}^{\prime}, \ldots, x_{m}^{\prime}, F_{1}^{*}(\vec{x}), \ldots, F_{i}^{*}(\vec{x})\right), \quad \text { if } j \notin S, \\ 0 \quad \text { if } j \in S,\end{array}\right.$

and these facts are expressible by sentences of $\widetilde{L}$ (which are therefore in $\widetilde{T}$ ).

Now to prove the first main theorem it suffices, by remarks in section 1 (see [1]), to show that if $\tilde{k}, \widetilde{K} \models \widetilde{T}, \tilde{k} \subseteq \widetilde{K}$ and $\chi$ is an existential sentence of $\widetilde{L}_{k}$ such that $\widetilde{K}^{+} \models \chi$, then $\tilde{k}^{+}=\chi$. We may also suppose here that $\chi$ has the form

$$
\exists x_{1}, \ldots, x_{r} \bigwedge_{s=1}^{n} \tau_{s}=0
$$

where each $\tau_{s}$ is either a term of $\bar{L}_{k}$ or else has the form $F_{i}\left(x_{i_{1}}, \ldots, x_{i_{m}}\right)-x_{i_{m+1}}$. This is because of standard logical equivalences and the facts that the formulas $x \neq y$ and $x<y$ are equivalent in $\bar{T}$ to the formulas $\exists z(y-x) \cdot z-1=0$ and $\exists z(y-x) \cdot z^{2}-1=0$ respectively and that composite terms may be unravelled 
by introducing new variables (e.g. replace $\tau(\sigma)=0$ by $\exists x(\sigma-x=0 \wedge \tau(x)=0)$ ). Now notice also that the formula

$$
F_{i}\left(y_{1}, \ldots, y_{m}\right)-y_{m+1}=0 \wedge y_{j} \geq 1
$$

(the $y_{i}$ 's being variables or constants) where $1 \leq j \leq m$ and $y_{j}$ is a variable, is equivalent in $\widetilde{T}$ to the formula

$$
\left(y_{j}>1 \wedge y_{m+1}=0\right) \vee\left(F_{i}\left(y_{1}, \ldots, y_{m}\right)\left(y_{j} / 1\right)-y_{m+1}=0 \wedge y_{j}=1\right),
$$

and that a similar equivalence holds with " $y_{j} \leq 0$ " in place of " $y_{j} \geq 1$ ". Thus by repeated use of all these equivalences we may suppose that $\chi$ actually has the form

$$
\exists x_{1}, \ldots, x_{r} \bigwedge_{s=1}^{n} \chi_{s}\left(x_{1}, \ldots, x_{r}\right),
$$

where each $\chi_{s}\left(x_{1}, \ldots, x_{s}\right)$ is either of the form $\tau\left(x_{1}, \ldots, x_{r}\right)=0$ for some term $\tau\left(x_{1}, \ldots, x_{r}\right)$ of $\bar{L}_{k}$ (i.e. a polynomial in $x_{1}, \ldots, x_{r}$ over $k$ ) or of the form

$$
\bigwedge_{j \notin S} 0<x_{i_{j}}<1 \wedge F_{i}\left(x_{i_{1}}^{\prime}, \ldots, x_{i_{m}}^{\prime}\right)-x_{i_{m+1}}=0
$$

for some $S \subseteq\{1, \ldots, m\}$, where $1 \leq i_{1}, \ldots, i_{m+1} \leq r$ and where

$$
x_{i_{j}}^{\prime}= \begin{cases}x_{i+j} & \text { for } j \notin S, \\ 0 \text { or } 1 & \text { for } j \in S .\end{cases}
$$

The proof of the first main theorem will be essentially by induction on the number of $\chi_{s}$ 's of this second form that occur in $\chi$ although it is convenient first to pad out the set of such $\chi_{s}$ 's. This the purpose of the following

2.1. Definition. Let $n, r \in \mathbb{N}$.

(i) A sequence $\left\langle\sigma_{1}, \ldots, \sigma_{n}\right\rangle$ of terms of $\widetilde{L}$ in the variables $x_{1}, \ldots, x_{r}$ is called an $(n, r)$-sequence if

(a) for $s=1, \ldots, n, \sigma_{s}$ has the form $F_{i}\left(y_{1}, \ldots, y_{m}\right)$ for some $i=1, \ldots, l$ and some $y_{1}, \ldots, y_{m} \in\left\{0,1, x_{1}, \ldots, x_{r}\right\}$, and

(b) if $1 \leq s \leq n, 1<i \leq l$ and $\sigma_{s}$ is $F_{i}\left(y_{1}, \ldots, y_{m}\right)$ (as in (a)), then $s>1$ and for some $t=1, \ldots, s-1, \sigma_{t}$ is $F_{i-1}\left(y_{1}, \ldots, y_{m}\right)$.

(ii) Those variables actually occurring in some term of an $(n, r)$-sequence $\vec{\sigma}$ are called $\vec{\sigma}$-bounded.

Clearly any $(n, r)$-sequence $\vec{\sigma}$ is also an $\left(n, r^{\prime}\right)$-sequence for any $r^{\prime} \geq r$ (and the set of $\vec{\sigma}$-bounded variables is the same), and any initial segment of an $(n, r)$ sequence is an $\left(n^{\prime}, r\right)$-sequence for the appropriate $n^{\prime} \leq n$. Further, any sequence satisfying (i) (a) may be clearly rearranged and padded out to an $\left(n^{\prime}, r\right)$-sequence for some $n^{\prime}$. Now let $\widetilde{K} \models \widetilde{T}$.

2.2. Definition. Suppose $\vec{\sigma}=\left\langle\sigma_{1}, \ldots, \sigma_{n}\right\rangle$ is an $(n, r)$-sequence. The natural domain of $\vec{\sigma}$ on $\widetilde{K}$, denoted $D^{r}(\vec{\sigma}, \widetilde{K})$, is defined to be $\prod_{i=1}^{r} I_{i}$ where

$$
I_{i}=\left\{\begin{array}{l}
\{x \in K: \widetilde{K} \models 0<x<1\} \quad \text { if } x_{i} \text { is } \vec{\sigma} \text {-bounded, } \\
K \text { otherwise. }
\end{array}\right.
$$

Clearly $D^{r}(\vec{\sigma}, \widetilde{K})$ is a definable open (in the sense of $\widetilde{K}$ ) subset of $K^{r}$.

Suppose now that $\tilde{k}=\widetilde{T}$ and $\tilde{k} \subseteq \widetilde{K}$. 
2.3. Definition. I denote by $M^{r}(\tilde{k}, \widetilde{K}, \vec{\sigma})$, where $\vec{\sigma}$ is an $(n, r)$-sequence, the ring of all those functions $f: D^{r}(\vec{\sigma}, \widetilde{K}) \rightarrow K$ for which there exists a polynomial $p\left(X_{1}, \ldots, X_{r}, Y_{1}, \ldots, Y_{n}\right) \in k\left[X_{1}, \ldots, X_{r}, Y_{1}, \ldots, Y_{n}\right]$ such that

$$
f(\vec{\alpha})=p\left(\vec{\alpha}, \sigma_{1}(\vec{\alpha}), \ldots, \sigma_{n}(\vec{\alpha})\right) \text { for all } \vec{\alpha} \in D^{r}(\vec{\sigma}, \widetilde{K}),
$$

where $\vec{\sigma}=\left\langle\sigma_{1}, \ldots, \sigma_{n}\right\rangle$.

The reductions preceding Definition 2.1 clearly imply

2.4. Lemma. In order to prove the main theorem it is sufficient to show that for all $\tilde{k}, \widetilde{K}=\widetilde{T}$ with $\tilde{k} \subseteq \widetilde{K}$, all $n, r \in \mathbb{N}$, all $(n, r)$-sequences $\vec{\sigma}$, and all $g_{1}, \ldots, g_{q} \in$ $M^{r}(\tilde{k}, \widetilde{K}, \vec{\sigma})$, if $g_{1}, \ldots, g_{q}$ have a common zero in $D^{r}(\vec{\sigma}, \widetilde{K})$, then they have one in $D^{r}(\vec{\sigma}, \tilde{k})$. (Note that we clearly have $D^{r}(\vec{\sigma}, \tilde{k}) \subseteq D^{r}(\vec{\sigma}, \widetilde{K})$.)

Of course our reductions show that the polynomials $p$ of (7) representing the $g_{i}$ 's of 2.4 may be taken to be either independent of the $Y_{i}$ 's or of the form $Y_{i}-X_{j}$ (for some $i=1, \ldots, n, j=1, \ldots, r$ ). However, while this observation will play a role later (in somewhat disguised form) it is much more convenient to work with rings of functions, and I now want to establish some elementary properties of these rings.

Fix, for the rest of this section, models $\tilde{k}, \widetilde{K}$ of $\widetilde{T}$ such that $\tilde{k} \subseteq \widetilde{K}$.

Suppose that $n, r \in \mathbb{N}$ and that $\vec{\sigma}=\left\langle\sigma_{1}, \ldots, \sigma_{n}\right\rangle$ is an $(n, r)$-sequence. Let $g \in$ $M^{r}(\tilde{k}, \widetilde{K}, \vec{\sigma})$. Then by (5) and the comments immediately following $(6), g$ is a $C^{\infty}$ function on $D^{r}(\vec{\sigma}, \widetilde{K})$ in the sense of $\widetilde{K}$. That is, for each $q \in \mathbb{N}, \widetilde{K}$ satisfies the usual $\varepsilon-\delta$ definition for the existence of continuous $q$ th partial derivatives of $g$ at all points of $D^{r}(\vec{\sigma}, \widetilde{K})$. Further, it clearly follows from (6) and (i)(b) of Definition 2.1 that these partial derivatives of $g$ all lie in $M^{r}(\tilde{k}, \widetilde{K}, \vec{\sigma})$. Thus $M^{r}(\tilde{k}, \widetilde{K}, \vec{\sigma})$ is a differential ring. It is also an integral domain. This is because $M^{r}(\widetilde{\mathbb{R}}, \widetilde{\mathbb{R}}, \vec{\sigma})$ is certainly an integral domain (since it is a ring of functions analytic on an open connected set) and this fact clearly transfers to $M^{r}(\widetilde{K}, \widetilde{K}, \vec{\sigma})$ (just represent elements of $M^{r}(\widetilde{K}, \widetilde{K}, \vec{\sigma})$ in the form (7) and quantify out the coefficients of $p)$, which contains $M^{r}(\tilde{k}, \widetilde{K}, \vec{\sigma})$ as a subring.

Suppose now that $p, q \leq r$ and $1 \leq i_{1}<\cdots<i_{q} \leq r$. For $g_{1}, \ldots, g_{p} \in$ $M^{r}(\tilde{k}, \widetilde{K}, \vec{\sigma})$ consider the (Jacobian) matrix

$$
\left(\begin{array}{ccc}
\frac{\partial g_{1}}{\partial x_{i_{1}}} & \cdots & \frac{\partial g_{1}}{\partial x_{i_{q}}} \\
\vdots & & \vdots \\
\frac{\partial g_{p}}{\partial x_{i_{1}}} & \cdots & \frac{\partial g_{p}}{\partial x_{i_{q}}}
\end{array}\right) .
$$

It is a matrix over $M^{r}(\tilde{k}, \widetilde{K}, \vec{\sigma})$ and I denote it by $\frac{\partial\left(g_{1}, \ldots, g_{p}\right)}{\partial\left(x_{i_{1}}, \ldots, x_{i_{q}}\right)}$. Note that if $p=q$, then

$$
\operatorname{det}\left(\frac{\partial\left(g_{1}, \ldots, g_{p}\right)}{\partial\left(x_{i_{1}}, \ldots, x_{i_{p}}\right)}\right) \in M^{r}(\tilde{k}, \widetilde{K}, \vec{\sigma}) .
$$

If $p=q=r$, I write $J\left(g_{1}, \ldots, g_{r}\right)$ for $\operatorname{det}\left(\frac{\partial\left(g_{1}, \ldots, g_{r}\right)}{\partial\left(x_{1}, \ldots, x_{r}\right)}\right)$.

2.5. Definition. Suppose $n, r \in \mathbb{N}$ and let $\vec{\sigma}$ be an $(n, r)$-sequence. Then a point $P \in K^{r}$ is called $(\tilde{k}, \vec{\sigma})$-definable if there exist $g_{1}, \ldots, g_{r} \in M^{r}(\tilde{k}, \widetilde{K}, \vec{\sigma})$ such that

(i) $P \in D^{r}(\vec{\sigma}, \widetilde{K})$ 
(ii) $g_{1}(P)=\cdots=g_{r}(P)=0$, and

(iii) $J\left(g_{1}, \ldots, g_{r}\right)(P) \neq 0$.

Examples. (D) Let $r \in \mathbb{N}$. Note that the empty sequence, $\varnothing$, is a $\langle 0, r\rangle$ sequence, that $D^{r}(\varnothing, \widetilde{K})=K^{r}$ and that $M^{r}(\tilde{k}, \widetilde{K}, \varnothing)$ may be identified with the polynomial ring $k\left[x_{1}, \ldots, x_{r}\right]$. Now suppose $P \in k^{r}$, say $P=\left\langle p_{1}, \ldots, p_{r}\right\rangle$. For $i=1, \ldots, r$ define $g_{i}\left(x_{1}, \ldots, x_{r}\right)=x_{i}-p_{i}$. Then $g_{1}, \ldots, g_{r} \in M^{r}(\tilde{k}, \widetilde{K}, \varnothing), g_{1}(P)=\cdots=$ $g_{r}(P)=0$ and $J\left(g_{1}, \ldots, g_{r}\right)(P)=1 \neq 0$. Hence $P$ is a $(\tilde{k}, \varnothing)$-definable point of $K^{r}$. Conversely, suppose $Q$ is a $(\tilde{k}, \varnothing)$-definable point of $K^{r}$. Then elementary algebra tells us that each coordinate of $Q$ is algebraic over (the field) $k$. Since $k$ is algebraically closed in $K$ (both being models of $\bar{T}$ ) it follows that $Q \in k^{r}$.

(E) More generally, suppose $n, r \in \mathbb{N}$ and that $\vec{\sigma}=\left\langle\sigma_{1}, \ldots, \sigma_{n}\right\rangle$ is an $(n, r)$ sequence. Let $s \geq 1$ and regard $\vec{\sigma}$ as an $(n, r+s)$-sequence. Then clearly $D^{r+s}(\vec{\sigma}, \widetilde{K})$ $=D^{r}(\vec{\sigma}, \widetilde{K}) \times K^{s}\left(\right.$ cf. 2.1(ii) and 2.2) and $M^{r+s}(\tilde{k}, \widetilde{K}, \vec{\sigma})$ may be identified with the polynomial $\operatorname{ring} M^{r}(\tilde{k}, \widetilde{K}, \vec{\sigma})\left[x_{r+1}, \ldots, x_{r+s}\right]$ over the domain $M^{r}(\tilde{k}, \tilde{K}, \vec{\sigma})$. Suppose $P \in D^{r}(\vec{\sigma}, \widetilde{K})$ and $Q \in K^{s}$ and that $\langle P, Q\rangle$ is $(\tilde{k}, \vec{\sigma})$-definable. Then elementary algebra again tells us that each coordinate of $Q$ is algebraic over the subfield $k\left(p_{1}, \ldots, p_{r}, \sigma_{1}(P), \ldots, \sigma_{n}(P)\right)$ of $K$ (where $\left.P=\left\langle p_{1}, \ldots, p_{r}\right\rangle\right)$.

Example (D) shows that a point of $K^{r}$ is $(\tilde{k}, \varnothing)$-definable if and only if it lies in $k^{r}$. In fact:

2.6. Main Lemma. For any $n, r \in \mathbb{N}$ and any $(n, r)$-sequence $\vec{\sigma}$, every $(\tilde{k}, \vec{\sigma})$ definable point of $K^{r}$ lies in $k^{r}$.

We shall also prove the following

2.7. Lemma. Let $n, r \in \mathbb{N}$ and let $\vec{\sigma}$ be an $(n, r)$-sequence. Suppose $g \in$ $M^{r}(\tilde{k}, \widetilde{K}, \vec{\sigma})$ and $g(P)=0$ for some $P \in D^{r}(\vec{\sigma}, \widetilde{K})$. Then for some $s \in \mathbb{N}$ there exists $Q_{0} \in D^{r}(\vec{\sigma}, \widetilde{K})$ and $Q_{1} \in K^{s}$ such that $g\left(Q_{0}\right)=0$ and $\left\langle Q_{0}, Q_{1}\right\rangle$ is $(\tilde{k}, \vec{\sigma})$ definable (cf. example (E) above).

Clearly the first main theorem follows from 2.4, 2.6 and 2.7 by taking the $g$ of 2.7 to be $\sum_{i=1}^{q} g_{i}^{2}$ with $g_{1}, \ldots, g_{q}$ as in 2.4 .

It is convenient to split the main lemma into two statements, the proofs of which are entirely different. They are:

2.8. Lemma. Suppose $n, r \in \mathbb{N}$ and that $\vec{\sigma}$ is an $(n, r)$-sequence. Suppose further that for each $s \geq r$ and each $(\tilde{k}, \vec{\sigma})$-definable point $\left\langle p_{1}, \ldots, p_{s}\right\rangle$ of $K^{s}$ there is some $B \in k$ such that $\widetilde{K} \models \bigwedge_{i=1}^{s}-B<p_{i}<B$. Then every $(\tilde{k}, \vec{\sigma})$-definable point of $K^{r}$ lies in $k^{r}$.

2.9. Lemma. Suppose that $n, r \in \mathbb{N}$ and that $\vec{\sigma}^{\prime}=\left\langle\sigma_{1}, \ldots, \sigma_{n}, \sigma_{n+1}\right\rangle$ is an $(n+$ $1, r)$-sequence. Let $\vec{\sigma}$ denote the $(n, r)$-sequence $\left\langle\sigma_{1}, \ldots, \sigma_{n}\right\rangle$. Suppose that for each $s \geq r$ every $(\tilde{k}, \vec{\sigma})$-definable point of $K^{s}$ lies in $k^{s}$. Then for each $s \geq r$ and each $\left(\tilde{k}, \vec{\sigma}^{\prime}\right)$-definable point $\left\langle p_{1}, \ldots, p_{s}\right\rangle$ of $K^{s}$, there is some $B \in k$ such that $\widetilde{K} \models$ $\bigwedge_{i=1}^{s}-B<p_{i}<B$.

Clearly the main lemma follows by induction on $n$ (for all values of $r$ ) from 2.8 and 2.9, the base step of the induction being provided by example (D).

We have now reduced the task of proving the first main theorem to that of proving Lemmas 2.7, 2.8 and 2.9. In fact, 2.7 and 2.8 require only minor modifications of the techniques developed in [16] but I prefer to deduce them from a general theory of 
Noetherian differential rings of definable functions which I shall develop in section 4 . I shall prove 2.7 and 2.8 in sections 5 and 7 respectively. These proofs do not depend on the fact that the $F_{i}$ 's have continuations to an open set containing $[0,1]^{m}$ and so we can deduce a modified model completeness result for unrestricted Pfaffian functions in situations where 2.9 holds trivially (e.g. when $\widetilde{K}$ is a cofinal extension of $\tilde{k}$ ) and I conclude section 7 with such a result. Section 8 is devoted to a proof of 2.9 which needs van den Dries's work on the model theory of finitely sub-analytic sets ([13]). I shall also rely heavily throughout most of this paper on Khovanskii's work on Pfaffian functions ([6]). The exact results needed from these two papers as well as some immediate corollaries are described in the next section.

\section{Results of Khovanskit and van Den Dries}

3.1. Proposition (Khovanskii [6]). Suppose that $h_{1}, \ldots, h_{l}$ is any Pfaffian chain of functions on $\mathbb{R}^{m+n}$. Suppose further that $g_{1}, \ldots, g_{m} \in \mathbb{R}\left[x_{1}, \ldots, x_{m+n}, h_{1}, \ldots, h_{l}\right]$ (where $x_{i}: \mathbb{R}^{m+n} \rightarrow \mathbb{R}$ denotes the ith projection function). Then there is a natural number $N$ such that for any $Q \in \mathbb{R}^{n}$ the set

$$
\begin{aligned}
& \left\{P \in \mathbb{R}^{m}: g_{1}(P, Q)=\cdots=g_{m}(P, Q)=0\right. \text { and } \\
& \left.\qquad \operatorname{det}\left(\frac{\partial\left(g_{1}, \ldots, g_{m}\right)}{\partial\left(x_{1}, \ldots, x_{m}\right)}\right)(P, Q) \neq 0\right\}
\end{aligned}
$$

contains at most $N$ elements.

The reader may have already observed that some such result has to be true if we are to have any chance of proving 2.6. In fact we need a version of 3.1 where $\mathbb{R}^{m+n}$ is replaced with sets of the form $\prod_{i=1}^{m+n} J_{i}$ where each $J_{i}$ is either $\mathbb{R}$ or $(0,1)$. That such a modification holds can be seen by inspecting Khovanskii's proof. Alternatively we may argue as follows.

Suppose $h_{1}, \ldots, h_{l}$ is a Pfaffian chain on $\prod_{i=1}^{m+n} J_{i}$. Define the functions $\alpha_{i}, \beta_{i}$ : $\mathbb{R}^{m+n} \rightarrow \mathbb{R}($ for $i=1, \ldots, m+n)$ by

$$
\begin{gathered}
\alpha_{i}(\vec{x})= \begin{cases}1 & \text { if } J_{i}=\mathbb{R}, \\
\frac{1}{\pi\left(1+x_{i}^{2}\right)} & \text { if } J_{i}=(0,1),\end{cases} \\
\beta_{i}(\vec{x})= \begin{cases}x_{i} & \text { if } J_{i}=\mathbb{R}, \\
\frac{1}{2}+\frac{1}{\pi} \cdot \tan ^{-1}\left(x_{i}\right) & \text { if } J_{i}=(0,1) .\end{cases}
\end{gathered}
$$

Then clearly the map $\vec{\beta}: \vec{x} \mapsto\left\langle\beta_{1}(\vec{x}), \ldots, \beta_{m+n}(\vec{x})\right\rangle$ is an analytic bijection from $\mathbb{R}^{m+n}$ to $\prod_{i=1}^{m+n} J_{i}$ so the functions $h_{i} \circ \vec{\beta}: \mathbb{R}^{m+n} \rightarrow \mathbb{R}$ (for $i=1, \ldots, l$ ) are defined and analytic throughout $\mathbb{R}^{m+n}$. Further, by the chain rule (and see also example B), the sequence $\alpha_{1}, \beta_{1}, \ldots, \alpha_{m+n}, \beta_{m+n}, h_{1} \circ \vec{\beta}, \ldots, h_{l} \circ \vec{\beta}$ is a Pfaffian chain on $\mathbb{R}^{m+n}$.

Let $M$ denote the ring of functions (defined on $\left.\prod_{i=1}^{m+n} J_{i}\right) \mathbb{R}\left[x_{1}, \ldots, x_{m+n}\right.$, $\left.h_{1}, \ldots, h_{l}\right]$ and $M^{*}$ the ring of functions (defined on $\mathbb{R}^{m+n}$ ) $\mathbb{R}\left[x_{1}, \ldots, x_{m+n}, \alpha_{1}, \ldots\right.$, $\left.\alpha_{m+n}, \beta_{1}, \ldots, \beta_{m+n}, h_{1} \circ \vec{\beta}, \ldots, h_{l} \circ \vec{\beta}\right]$. Suppose $g_{1}, \ldots, g_{m} \in M, P \in \prod_{i=1}^{m} J_{i}$, $Q \in \prod_{i=m+1}^{m+n} J_{i}, g_{1}(P, Q)=\cdots=g_{m}(P, Q)=0$ and $\operatorname{det}\left(\frac{\partial\left(g_{1}, \ldots, g_{m}\right)}{\partial\left(x_{1}, \ldots, x_{m}\right)}\right)(P, Q) \neq 0$. Then clearly $g_{1} \circ \vec{\beta}, \ldots, g_{m} \circ \vec{\beta} \in M^{*}$ and $g_{1} \circ \vec{\beta}\left(P^{\prime}, Q^{\prime}\right)=\cdots=g_{m} \circ \vec{\beta}\left(P^{\prime}, Q^{\prime}\right)=0$ 
where $\left\langle P^{\prime}, Q^{\prime}\right\rangle=\vec{\beta}^{-1}(P, Q)$. Further, as an easy calculation using the chain rule shows, we have

$$
\begin{aligned}
\frac{\partial\left(g_{1} \circ \vec{\beta}, \ldots, g_{m} \circ \vec{\beta}\right)}{\partial\left(x_{1}, \ldots, x_{m}\right)}\left(P^{\prime}, Q^{\prime}\right)= & \frac{\partial\left(g_{1}, \ldots, g_{m}\right)}{\partial\left(x_{1}, \ldots, x_{m}\right)}(P, Q) \\
& \times \frac{\partial\left(\beta_{1}, \ldots, \beta_{m}\right)}{\partial\left(x_{1}, \ldots, x_{m}\right)}\left(P^{\prime}, Q^{\prime}\right) .
\end{aligned}
$$

Now

$$
\operatorname{det}\left(\frac{\partial\left(\beta_{1}, \ldots, \beta_{m}\right)}{\partial\left(x_{1}, \ldots, x_{m}\right)}\right)\left(P^{\prime}, Q^{\prime}\right)=\prod_{i=1}^{m} \alpha_{i}\left(P^{\prime}, Q^{\prime}\right) \neq 0,
$$

so the left hand side of $(*)$ has non-zero determinant. Since $P^{\prime}$ depends only on $P$ and $Q^{\prime}$ only on $Q$ we can now use 3.1 to conclude:

3.2. Corollary. Proposition 3.1 holds with $\prod_{i=1}^{m+n} J_{i}$ in place of $\mathbb{R}^{m+n}$ where each $J_{i}$ is either $\mathbb{R}$ or $(0,1)$.

The fact that the upper bound $N$ is independent of $Q$ here can now be used to transfer this result to the situation we are interested in. The easy formal details required for the proof of the following result are left to the reader.

3.3. Corollary. Suppose $n, r_{1}, r_{2} \in \mathbb{N}$ and that $\vec{\sigma}$ is an $\left(n, r_{1}+r_{2}\right)$-sequence. Suppose further that $\tilde{k}, \widetilde{K} \models \widetilde{T}, \tilde{k} \subseteq \widetilde{K}$, and that $g_{1}, \ldots, g_{r_{1}} \in M^{r_{1}+r_{2}}(\tilde{k}, \widetilde{K}, \vec{\sigma})$. Then there is $N \in \mathbb{N}$ such that for each $Q \in K^{r_{2}}$ the set

$$
\begin{gathered}
\left\{P \in K^{r_{1}}:\langle P, Q\rangle \in D^{r_{1}+r_{2}}(\vec{\sigma}, K), g_{1}(P, Q)=\cdots=g_{r_{1}}(P, Q)=0\right. \\
\text { and } \left.\operatorname{det}\left(\frac{\partial\left(g_{1}, \ldots, g_{r_{1}}\right)}{\partial\left(x_{1}, \ldots, x_{r_{1}}\right)}\right)(P, Q) \neq 0\right\}
\end{gathered}
$$

contains at most $N$ elements.

I now turn to a result of van den Dries concerning sets and functions definable in the structure $\mathbb{R}_{a n}$ (cf. section 1). The result we need can be found in [13] where it is formulated in terms of so-called "finitely sub-analytic sets". Since these are exactly the sets definable in $\mathbb{R}_{a n}$ (see [3]) we may reformulate the result as follows

3.4. Proposition (van den Dries [13]). (i) $\mathbb{R}_{a n}$ is 0-minimal (i.e. every subset of $\mathbb{R}$ definable (with parameters) in $\mathbb{R}_{a n}$ is a finite union of open intervals and points).

(ii) If $e \in \mathbb{R}$ and $f:(e, \infty) \rightarrow \mathbb{R}$ is any function definable (with parameters) in $\mathbb{R}_{\text {an }}$, then there exists $d \geq e$ such that on $(d, \infty)$ the function $f$ may be represented by a convergent Puiseux expansion:

$$
f(x)=\sum_{i=p}^{\infty} a_{i} \cdot x^{-i / q}
$$

where $q \in \mathbb{N}, q \geq 1, p \in \mathbb{Z}, a_{i} \in \mathbb{R}$ (for $i \in \mathbb{Z}, i \geq p$ ) and $a_{p} \neq 0$ provided $f$ is not (eventually) identically zero.

Now, as pointed out in section 1 , every subset of $\mathbb{R}^{n}$ (for any $n$ ) definable in the structure $\widetilde{\mathbb{R}}$ is definable in $\mathbb{R}_{\text {an }}$. Hence 3.4 holds with $\widetilde{\mathbb{R}}$ in place of $\mathbb{R}_{a n}$. I need the following consequence of this fact. 
3.5. Corollary. Suppose $\widetilde{K} \models \widetilde{T}, e \in K$ and $g:(e, \infty) \rightarrow K$ is a $\widetilde{K}$ definable function which is not eventually identically zero. Then there is a rational number $s$ and a non-zero element $a \in K$ such that $g(x) x^{s} \rightarrow a$ as $x \rightarrow \infty$ (in the sense of $\widetilde{K})$.

Proof. Suppose $\phi(\vec{b}, x, y)$ defines the graph of $g$ in $\widetilde{K}$ where $\phi(\vec{z}, x, y)$ is an $\widetilde{L}$ formula. Let $\psi(\vec{z})$ be the $\widetilde{L}$-formula

$$
\exists u(\forall x>u \exists ! y \phi(\vec{z}, x, y) \wedge \forall x>u \exists w>x \neg \phi(\vec{z}, w, 0)),
$$

and note that $\widetilde{K} \models \psi(\vec{b})$.

Now suppose that $\vec{\alpha}$ is a tuple of reals such that $\widetilde{\mathbb{R}} \models \psi(\vec{\alpha})$ and let $f_{\vec{\alpha}}:(\beta, \infty) \rightarrow$ $\mathbb{R}$ be the function defined by $\phi(\vec{\alpha}, x, y)$ in $\widetilde{\mathbb{R}}$ (for suitable $\beta \in \mathbb{R}$ ). By $(3.4)$ (ii) $f_{\vec{\alpha}}$ may be represented in the form $(*)$ for sufficiently large $x$, and we clearly have $a_{p} \neq 0$ and $f_{\vec{\alpha}}(x) x^{p / q} \rightarrow a_{p}$ as $x \rightarrow \infty$.

Now by elementary real analysis the series $(*)$ may be differentiated term by term to obtain the convergent representation

$$
f_{\vec{\alpha}}^{\prime}(x)=\sum_{i=p}^{\infty}-\frac{i a_{i}}{q} x^{(-i / q)-1}
$$

(for sufficiently large $x \in \mathbb{R}$ ), and we have that $f_{\vec{\alpha}}^{\prime}(x) x^{(p / q)+1} \rightarrow-\frac{p a_{p}}{q}$ as $x \rightarrow \infty$. It follows that $\lim _{x \rightarrow \infty}-\left(f_{\vec{\alpha}}^{\prime}(x) x\right) /\left(f_{\vec{\alpha}}(x)\right)$ exists and equals $p / q$. By using the usual $\varepsilon-\delta$ definition of derivatives and limits we may clearly write down an $\widetilde{L}$-formula $\chi(\vec{z}, y)$ expressing (in $\widetilde{\mathbb{R}}):$ : $\psi(\vec{z})$ and $\lim _{x \rightarrow \infty}-\left(f_{\vec{z}}^{\prime}(x) x\right) / f_{\vec{z}}(x)=y$." We have shown that the $\widetilde{L}$-formula $\exists \vec{z} \chi(\vec{z}, y)$ defines in $\widetilde{\mathbb{R}}$ a set of rationals, and since, by the comments above, $\widetilde{\mathbb{R}}$ is a 0 -minimal structure, it follows that this set is finite, say $\left\{s_{1}, \ldots, s_{n}\right\}$. We have also shown that the $\widetilde{L}$-sentence expressing: $\forall \vec{z}(\psi(\vec{z}) \rightarrow$ $\bigvee_{i=1}^{n}\left(\lim _{x \rightarrow \infty} f_{\vec{z}}(x) \cdot x^{s_{i}}\right.$ exists and is non-zero) $) "$ is true in $\widetilde{\mathbb{R}}$, and hence in $\widetilde{K}$. Since $\widetilde{K} \models \psi(\vec{b})$ and $f_{\vec{b}}=g$ (eventually in $\widetilde{K}$ ) the result follows.

\section{Differentiable Germs in arbitrary eXPansions of $\overline{\mathbb{R}}$}

Throughout this section $\overline{\overline{\mathbb{R}}}$ denotes any expansion of the ordered field $\overline{\mathbb{R}}, \overline{\bar{L}}$ its language and $\overline{\bar{T}}$ its theory. We employ conventions analogous to those set out at the beginning of section 2 concerning models of $\overline{\bar{T}}$.

Let $\overline{\bar{K}}=\overline{\bar{T}}$. As we have already seen many local notions from topology and calculus can be immediately transferred from $\overline{\overline{\mathbb{R}}}$ to $\overline{\bar{K}}$ and I will assume the reader is familiar with this process. It should always be clear how (and where) these notions are to be interpreted. The implicit function theorem, however, requires some comment.

Suppose $r, m \in \mathbb{N}, r, m \geq 1$ and $\langle P, Q\rangle=\left\langle p_{1}, \ldots, p_{r}, q_{1}, \ldots, q_{m}\right\rangle \in K^{r+m}$. Let $U$ be a definable (i.e. $K$-definable with parameters) open neighbourhood of $\langle P, Q\rangle$ and suppose $f_{1}, \ldots, f_{m}: U \rightarrow K$ are definable functions which are infinitely differentiable throughout $U$. Suppose further that $\langle P, Q\rangle$ is a non-singular zero of $f_{1}, \ldots, f_{m}$ with respect to $x_{r+1}, \ldots, x_{r+m}$. This means, by definition, that 
$f_{i}(P, Q)=0$ for $i=1, \ldots, m$ and that the determinant of the Jacobian matrix

$$
\Delta=\Delta\left(x_{1}, \ldots, x_{r+m}\right)=\left(\begin{array}{ccc}
\frac{\partial f_{1}}{\partial x_{r+1}} & \cdots & \frac{\partial f_{1}}{\partial x_{r+m}} \\
\vdots & & \vdots \\
\frac{\partial f_{m}}{\partial x_{r+1}} & \cdots & \frac{\partial f_{m}}{\partial x_{r+m}}
\end{array}\right)
$$

does not vanish at $\langle P, Q\rangle$.

Now if $\overline{\bar{K}}=\overline{\overline{\mathbb{R}}}$ we can apply the implicit function theorem (see e.g. [4]) to obtain open neighbourhoods $V_{1}$ of $P$ (in $K^{r}$ ) and $V_{2}$ of $Q$ (in $K^{m}$ ) such that

4.1. $V_{1} \times V_{2} \subseteq U$.

4.2. For each $\vec{x} \in V_{1}$ there is a unique point $\left\langle y_{1}, \ldots, y_{m}\right\rangle=\left\langle y_{1}(\vec{x}), \ldots, y_{m}(\vec{x})\right\rangle \in V_{2}$ such that $f_{i}(\vec{x}, \vec{y})=0$ for $i=1, \ldots, m$, and this point satisfies $J(\vec{x}, \vec{y}) \neq 0$.

4.3. The functions $y_{i}: V_{1} \rightarrow K$ (for $i=1, \ldots, m$ ) are infinitely differentiable and for each $l=1, \ldots, r$ and $\vec{x} \in V_{1}$

$$
\left(\begin{array}{c}
\frac{\partial y_{1}}{\partial x_{l}} \\
\vdots \\
\frac{\partial y_{m}}{\partial x_{l}}
\end{array}\right)=-\Delta^{-1}\left(\begin{array}{c}
\frac{\partial f_{1}}{\partial x_{l}} \\
\vdots \\
\frac{\partial f_{m}}{\partial x_{l}}
\end{array}\right)
$$

where the right hand side is evaluated at the point $\left\langle\vec{x}, y_{1}(\vec{x}), \ldots, y_{m}(\vec{x})\right\rangle$.

We require 4.1-4.3 to hold for arbitrary $\overline{\bar{K}}$ and that this is the case can be argued as follows. Firstly, the existence of $V_{1}$ and $V_{2}$ satisfying 4.1 and 4.2 can be guaranteed since we may suppose they are box neighbourhoods (i.e. of the form $\left\{\left\langle z_{1}, \ldots, z_{t}\right\rangle \in K^{t}:\left|\alpha_{i}-z_{i}\right|<\varepsilon\right.$ for $\left.i=1, \ldots, t\right\}$ for some $\alpha_{1}, \ldots, \alpha_{t}, \varepsilon \in K$ with $\varepsilon>0$ ). Having fixed such $V_{1}$ and $V_{2}$ the uniqueness in 4.2 guarantees that the $y_{i}$ 's are definable functions which, by transfer, are continuously differentiable throughout $V_{1}$ and satisfy the formula in 4.3. But this formula implies (simply by arguing in $\overline{\bar{K}}$ ) that the $y_{i}$ 's are infinitely differentiable throughout $V_{1}$.

I now turn to germs of differentiable definable functions in (an arbitrary given model of $\overline{\bar{T}}) \overline{\bar{K}}$.

4.4. Definition. Let $n \in \mathbb{N}, n \geq 1$.

(i) A neighbourhood system (n.s.) in $K^{n}$ is a non-empty collection of non-empty, definable open subsets of $K^{n}$ which is closed under (finite) intersection.

(ii) For $\mathfrak{G}$ a n.s. in $K^{n}, \mathfrak{D}^{(n)}(\mathfrak{G})^{-}$denotes the set of all pairs $\langle f, U\rangle$ where $U \in \mathfrak{G}$ and $f: U \rightarrow K$ is an infinitely differentiable definable function.

(iii) For $\left\langle f_{1}, U_{1}\right\rangle,\left\langle f_{2}, U_{2}\right\rangle \in \mathfrak{D}^{(n)}(\mathfrak{G})^{-},\left\langle f_{1}, U_{1}\right\rangle \sim\left\langle f_{2}, U_{2}\right\rangle$ means that there is some $U \in \mathfrak{G}$ with $U \subseteq U_{1} \cap U_{2}$ such that $f_{1}(\vec{x})=f_{2}(\vec{x})$ for all $\vec{x} \in U$. This is clearly an equivalence relation and the equivalence class of $\langle f, U\rangle\left(\in \mathfrak{D}^{(n)}(\mathfrak{G})^{-}\right)$is denoted $[f, U]$.

(iv) The set of equivalence classes, or germs, is denoted $\mathfrak{D}^{(n)}(\mathfrak{G})$.

Clearly $\mathfrak{D}^{(n)}(\mathfrak{G})$ is naturally a differential (unital) ring and I continue to write $\frac{\partial}{\partial x_{1}}, \ldots, \frac{\partial}{\partial x_{n}}$ for the obvious induced derivatives on $\mathfrak{D}^{(n)}(\mathfrak{G})$.

4.5. Lemma. Let $n \in \mathbb{N}, n \geq 1$ and suppose $\mathfrak{G}$ is a n.s. in $K^{n}$. Suppose further that $M$ is a subring of $\mathfrak{D}^{(n)}(\mathfrak{G})$ closed under differentiation and that $I$ is a finitely generated ideal of $M$ also closed under differentiation. Let $\left\{\left[g_{1}, U_{1}\right], \ldots,\left[g_{s}, U_{s}\right]\right\}$ 
be any finite set of generators for $I$ and set $Z=\left\{P \in \bigcap_{i=1}^{s} U_{i}: g_{i}(P)=0\right.$ for $i=1, \ldots, s\}$. Then for some $U \in \mathfrak{G}, U \cap Z$ is an open (definable) subset of $K^{n}$.

Proof. Since $I$ is closed under differentiation and $\mathfrak{G}$ under finite intersection there exist $U \in \mathfrak{G}$ and definable functions $a_{i, j}^{(r)}(1 \leq i, j \leq s, 1 \leq r \leq n)$ such that $g_{1}, \ldots, g_{s}$ and the $a_{i, j}^{(r)}$,s all have domains containing $U$, are infinitely differentiable throughout $U$, and satisfy the equations

$$
\frac{\partial g_{i}}{\partial x_{r}}=\sum_{j=1}^{s} a_{i, j}^{(r)} \cdot g_{j} \quad(1 \leq i \leq s, 1 \leq r \leq n)
$$

on $U$.

I claim that $U \cap Z$ is open in $K^{n}$. For suppose $P=\left\langle p_{1}, \ldots, p_{n}\right\rangle \in U \cap Z$ and let $U_{0}$ be an open box neighbourhood of $P$ contained in $U$. It suffices to show that each $g_{i}$ vanishes on $U_{0}$ so suppose that this is not the case. Since each $g_{i}$ certainly vanishes at $P$ we can clearly find $Q, S \in U_{0}$ such that $g_{i}(Q)=0$ for all $i=1, \ldots, s$ and $g_{i}(S) \neq 0$ for some $i=1, \ldots, s$ and such that $Q$ and $S$ differ in exactly one coordinate, which we suppose for convenience is the first. Say $Q=\left\langle q_{1}, q_{2}, \ldots, q_{n}\right\rangle$ and $S=\left\langle q_{1}^{\prime}, q_{2}, \ldots, q_{n}\right\rangle$ where $q_{1} \neq q_{1}^{\prime}$. Let $(a, b)$ be an open interval in $K$ such that $q_{1}, q_{2}^{\prime} \in(a, b)$ and $(a, b) \times\left\{\left\langle q_{2}, \ldots, q_{n}\right\rangle\right\} \subseteq U_{0}$. For any definable function $f: U_{0} \rightarrow K$ let $\bar{f}$ be the result of substituting $q_{i}$ for $x_{i}$ in $f$ for $i=2, \ldots, n$. Then by $(*)$ (for $r=1$ ) we have

$$
\left(\begin{array}{c}
\bar{g}_{1}^{\prime} \\
\vdots \\
\bar{g}_{s}^{\prime}
\end{array}\right)=A\left(\begin{array}{c}
\bar{g}_{1} \\
\vdots \\
\bar{g}_{s}
\end{array}\right)
$$

for all $x_{1} \in(a, b)$, where $A$ is the matrix $\left.\overline{\left(\overline{a_{i, j}^{(1)}}\right.}\left(x_{1}\right)\right)_{1 \leq i, j \leq s}$ and where' denotes $\frac{d}{d x_{1}}$.

We now transfer this situation to $\overline{\overline{\mathbb{R}}}$ (by quantifying out parameters) and obtain a real interval $(c, d)$, continuously differentiable functions $h_{i}, b_{i, j}:(c, d) \rightarrow \mathbb{R}$ (for $1 \leq i, j \leq s)$ and points $\alpha, \beta \in(c, d)$ such that (setting $B=\left(b_{i, j}(x)\right)_{1 \leq i, j \leq s}$ )

$$
\begin{gathered}
\left(\begin{array}{c}
h_{1}^{\prime} \\
\vdots \\
h_{s}^{\prime}
\end{array}\right)=B\left(\begin{array}{c}
h_{1} \\
\vdots \\
h_{s}
\end{array}\right) \quad \text { for all } x \in(c, d), \\
h_{i}(\alpha)=0 \text { for all } i=1, \ldots, s,
\end{gathered}
$$

and

$$
h_{i}(\beta) \neq 0 \quad \text { for some } i=1, \ldots, s .
$$

The theory of linear differential equations (see e.g. [9], Theorem 11.4.1 and its proof) now tells us that for all $x \in(c, d)$

$$
\left(\begin{array}{c}
h_{1}(x) \\
\vdots \\
h_{s}(x)
\end{array}\right)=E(x)^{-1} E(\alpha)\left(\begin{array}{c}
h_{1}(\alpha) \\
\vdots \\
h_{s}(\alpha)
\end{array}\right)
$$

for some $s \times s$ matrix $E$ of functions on $(c, d)$ which is invertible for all $x \in(c, d)$. Setting $x=\beta$ here gives the required contradiction. 
4.6. Notation. For $n \in \mathbb{N}, n \geq 1$ and $P \in K^{n}, \mathfrak{G}_{P}$ denotes the set of all definable open neighbourhoods of $P$. It is clearly a n.s. in $K^{n}$. Write $\mathfrak{D}^{(n)}(P)^{-}$and $\mathfrak{D}^{(n)}(P)$ for $\mathfrak{D}^{(n)}\left(\mathfrak{G}_{P}\right)^{-}$and $\mathfrak{D}^{(n)}\left(\mathfrak{G}_{P}\right)$ respectively. If $g \in \mathfrak{D}^{(n)}(P)$, say $g=[f, U], g(P)$ denotes the element $f(P)$ of $K$. It is clearly well defined. Finally, depending on convenience, $d_{P} g$ or $d_{P} f$ denotes $\left\langle\frac{\partial f}{\partial x_{1}}(P), \ldots, \frac{\partial f}{\partial x_{n}}(P)\right\rangle$ considered as an element of the $K$-vector space $K^{n}$.

I now wish to return to the situation of 4.1-4.3 to discuss some classical results applied to the present context. So let $r, m, P, Q, f_{1}, \ldots, f_{m}, U$ be as in the discussion of the implicit function theorem at the beginning of this section. Let $n=r+m$. Define $\phi_{1}, \ldots, \phi_{n}$ by $\phi_{i}(\vec{x})=x_{i}$ for $i=1, \ldots, r$, and $\phi_{i}(\vec{x})=y_{i-r}(\vec{x})$ for $i=r+1, \ldots, n$ (cf. 4.2, 4.3) where $\vec{x}=\left\langle x_{1}, \ldots, x_{r}\right\rangle$. These functions are defined and are infinitely differentiable on a set in $\mathfrak{G}_{P}$ (namely $V_{1}$ ) and hence determine germs in $\mathfrak{D}^{(r)}(P)$. Notice also that $\left\langle\phi_{1}(P), \ldots, \phi_{n}(P)\right\rangle=\langle P, Q\rangle$ so we have an induced mapping ^: $\mathfrak{D}^{(n)}(P, Q) \rightarrow \mathfrak{D}^{(r)}(P)$ defined (on functions) by $\hat{f}(\vec{x})=f\left(\phi_{1}(\vec{x}), \ldots, \phi_{n}(\vec{x})\right), \vec{x} \in$ $W^{\prime}$, where $\langle f, W\rangle \in \mathfrak{D}^{(n)}(P, Q)^{-}$and $W^{\prime}=\left\{\vec{x} \in V_{1}:\left\langle\phi_{1}(\vec{x}), \ldots, \phi_{n}(\vec{x})\right\rangle \in W\right\}$. (Clearly $W^{\prime} \in \mathfrak{G}_{P}$.) This mapping is clearly a (unital) ring homomorphism and its kernel consists exactly of those germs $[f, W]$ such that $f$ vanishes on $V \cap Z$ for some $V \in \mathfrak{G}_{P, Q}$ (with $V \subseteq W$ ), where $Z=\left\{\left\langle x_{1}, \ldots, x_{n}\right\rangle \in U: f_{i}\left(x_{1}, \ldots, x_{n}\right)=0\right.$ for $i=1, \ldots, m\}$. In particular $\left[\widehat{f_{i}, U}\right]=0\left(\right.$ in $\left.\mathfrak{D}^{(r)}(P)\right)$ for $i=1, \ldots, m$, so $\frac{\partial \hat{f}_{i}}{\partial x_{j}}=0$ (in $\left.\mathfrak{D}^{(r)}(P)\right)$ for $i=1, \ldots, m$ and $j=1, \ldots, r$.

4.7. Lemma. With the above notation we have that for all $g \in \mathfrak{D}^{(n)}(P, Q)$, the sequence of vectors $d_{P, Q} f_{1}, \ldots, d_{P, Q} f_{m}, d_{P, Q} g$ is linearly independent over $K$ if and only if $d_{P} \hat{g} \neq 0\left(\right.$ in $\left.K^{r}\right)$.

Proof. Notice first that the sequence $d_{P, Q} f_{1}, \ldots, d_{P, Q} f_{m}$ is certainly linearly independent since $J(P, Q) \neq 0$ (cf. the discussion at the beginning of this section). Write $g=\left[f_{m+1}, W\right]$.

Suppose that $\sum_{i=1}^{m+1} a_{i} \cdot d_{P, Q} f_{i}=0$ with not all the $a_{i}$ 's zero. Then $a_{m+1} \neq 0$. By the chain rule

$$
\frac{\partial \hat{f}_{i}}{\partial x_{j}}(P)=\sum_{l=1}^{n} \frac{\partial f_{i}}{\partial x_{l}}(P, Q) \cdot \frac{\partial \phi_{l}}{\partial x_{j}}(P)
$$

for $j=1, \ldots, r, i=1, \ldots, m+1$. Now by the remark before the lemma, our assumption and $(*)$ we have

$$
\begin{aligned}
\frac{\partial \hat{f}_{m+1}}{\partial x_{j}}(P) & =a_{m+1}^{-1} \sum_{i=1}^{m+1} a_{i} \frac{\partial \hat{f}_{i}}{\partial x_{j}}(P) \\
& =a_{m+1}^{-1} \cdot \sum_{l=1}^{n}\left(\frac{\partial \phi_{l}}{\partial x_{j}}(P) \sum_{i=1}^{m+1} a_{i} \frac{\partial f_{i}}{\partial x_{l}}(P, Q)\right)=0
\end{aligned}
$$

for $j=1, \ldots, r$, as required.

Suppose now that the sequence $d_{P, Q} f_{1}, \ldots, d_{P, Q} f_{m+1}$ is linearly independent. Let $A$ denote the $n \times(m+1)$ matrix (over $K$ ) with columns $d_{P, Q} f_{i}$ for $1 \leq i \leq m+1$. Then $A$ determines a $K$-linear map from $K^{n}$ onto $K^{m+1}$ with kernel of dimension $n-(m+1)=r-1$. Moreover, by $(*)$ and the remark before the lemma

$$
\left\langle\frac{\partial \phi_{1}}{\partial x_{j}}(P), \ldots, \frac{\partial \phi_{n}}{\phi x_{j}}(P)\right\rangle A=\left\langle 0, \ldots, 0, \frac{\partial \hat{f}_{m+1}}{\partial x_{j}}(P)\right\rangle \quad \text { for } j=1, \ldots, r \text {. }
$$


But the sequence of vectors $\left\langle\left\langle\frac{\partial \phi_{1}}{\partial x_{j}}(P), \ldots, \frac{\partial \phi_{n}}{\partial x_{j}}(P)\right\rangle: 1 \leq j \leq r\right\rangle$ is linearly independent (since $\frac{\partial \phi_{i}}{\partial x_{j}}=\delta_{i, j}$ for $1 \leq i, j \leq r$ ), so not all of them are in $\operatorname{Ker}(A)$. Thus $\frac{\partial \hat{f}_{m+1}}{\partial x_{j}}(P) \neq 0$ for some $j=1, \ldots, r$, as required.

4.8. Definition. Let $n, s \in \mathbb{N}, n \geq 1$. Suppose $g_{1}, \ldots, g_{s}$ are infinitely differentiable definable functions with domains open in $K^{n}$. Then

$$
V\left(g_{1}, \ldots, g_{s}\right) \stackrel{\text { def }}{=}\left\{Q \in \bigcap_{i=1}^{s} \operatorname{dom}\left(g_{i}\right): g_{i}(Q)=0 \text { for } i=1, \ldots, s\right\}
$$

and

$V^{n s}\left(g_{1}, \ldots, g_{s}\right) \stackrel{\text { def }}{=}\left\{Q \in V\left(g_{1}, \ldots, g_{s}\right):\left\langle d_{Q} g_{i}: 1 \leq i \leq s\right\rangle\right.$ is linearly independent $\}$.

(For $s=0, V=V^{n s}=K^{n}$.)

The following theorem will be used repeatedly throughout this paper.

4.9. Theorem. Let $n \in \mathbb{N}, n \geq 1, P_{0} \in K^{n}$, and suppose $M$ is a Noetherian (unital) subring of $\mathfrak{D}^{(n)}\left(P_{0}\right)$ closed under differentiation. Let $m \in \mathbb{N}$ and suppose $\left[f_{i}, U_{i}\right] \in M$ for $i=1, \ldots, m$. Suppose further that $P_{0} \in V^{n s}\left(f_{1}, \ldots, f_{m}\right)$. Then one of the following is true:

(i) $n=m$, or

(ii) $m<n$ and for any $[h, W] \in M$ with $h\left(P_{0}\right)=0, h$ vanishes on $U \cap$ $V^{n s}\left(f_{1}, \ldots, f_{m}\right)$ for some $U \in \mathfrak{G}_{P_{0}}($ with $U \subseteq W)$, or

(iii) $m<n$ and for some $[h, W] \in M, P_{0} \in V^{n s}\left(f_{1}, \ldots, f_{m}, h\right)$.

Proof. If $m \neq n$, then $m<n$ since $P_{0} \in V^{n s}\left(f_{1}, \ldots, f_{m}\right)$. Say $r+m=n$ where $1 \leq r \leq n$.

Now since $\left\langle d_{P_{0}} f_{i}: 1 \leq i \leq m\right\rangle$ is linearly independent there exists an $m$-element subset of $\{1, \ldots, n\}, S$ say, such that the matrix $\left(\frac{\partial f_{i}}{\partial x_{j}}\left(P_{0}\right)\right)_{1 \leq i \leq m, j \in S}$ is non-singular. There is no harm here in supposing that $S=\{r+1, \ldots, n\}$, so if we denote by $\lambda$ the function

$$
\left\langle x_{1}, \ldots, x_{n}\right\rangle \mapsto \operatorname{det}\left(\frac{\partial f_{i}}{\partial x_{j}}\left(x_{1}, \ldots, x_{n}\right)\right)_{1 \leq i \leq m, r+1 \leq j \leq n},
$$

then clearly $\left[\lambda, U_{0}\right] \in M$ (for some $\left.U_{0} \in \mathfrak{G}_{P_{0}}\right)$ and $\left[\lambda, U_{0}\right](=\Lambda$, say) is invertible in $\mathfrak{D}^{(n)}\left(P_{0}\right)$. Let $M^{*}=M\left[\Lambda^{-1}\right]$. Now write $P_{0}$ as $\langle P, Q\rangle$, where $P \in K^{r}$ and $Q \in K^{m}$, and consider the map ^ $: \mathfrak{D}^{(n)}(P, Q) \rightarrow \mathfrak{D}^{(r)}(P)$ described above. Clearly $\widehat{M}^{*}$, the image of $M^{*}$ under ${ }^{\wedge}$, is a Noetherian (unital) subring of $\mathfrak{D}^{(r)}(P)$ and is closed under differentiation. This latter fact follows easily from the chain rule and 4.3 (this is why we consider $M^{*}$ - the entries of $\Delta^{-1}$ (in 4.3) determine germs in $M^{*}$, but not necessarily in $\left.M\right)$. Now let $I$ denote the ideal $\left\{g \in \widehat{M}^{*}: g(P)=0\right\}$ of $\widehat{M}^{*}$.

Case 1. $I=\{0\}$. Suppose $[h, W] \in M$ and $h\left(P_{0}\right)=0$. Let $g=[h, W]$. Then $g\left(P_{0}\right)=0$ so $\hat{g}(P)=0$, i.e. $\hat{g} \in I$. Hence $\hat{g}=0$ in $\mathfrak{D}^{(r)}(P)$. The conclusion of (ii) in the statement of the theorem now follows from the comments before 4.7 .

Case 2. $I \neq\{0\}$. Since $I$ is finitely generated it clearly follows from 4.5 (with $\mathfrak{G}=\mathfrak{G}_{P}, M=\widehat{M}^{*}$ ) that $I$ is not closed under differentiation. Hence there is some 
$g \in M^{*}$ such that $\hat{g} \in I$, i.e. $\hat{g}(P)=0$ so $g\left(P_{0}\right)=0$, and some $i$ with $1 \leq i \leq r$ such that

$$
\frac{\partial \hat{g}}{\partial x_{i}} \notin I, \quad \text { i.e. } \frac{\partial \hat{g}}{\partial x_{i}}(P) \neq 0 .
$$

Now for some $s \in \mathbb{N}, \Lambda^{s} \cdot g \in M$. Let $f=\Lambda^{s} \cdot g$. Then $f\left(P_{0}\right)=0$ and, further,

$$
\begin{aligned}
\frac{\partial \hat{f}}{\partial x_{i}}(P) & =\left(s \hat{\Lambda}^{s-1} \cdot \frac{\partial \hat{\Lambda}}{\partial x_{i}} \cdot \hat{g}\right)(P)+\left(\hat{\Lambda}^{s} \cdot \frac{\partial \hat{g}}{\partial x_{i}}\right)(P) \\
& =\hat{\Lambda}^{s}(P) \cdot \frac{\partial \hat{g}}{\partial x_{i}}(P) \neq 0 .
\end{aligned}
$$

Thus $d_{P} \hat{f} \neq 0$ and hence, by 4.7 , the conclusion of (iii) in the statement of the theorem holds (with $[h, W]=f$ ).

Before leaving this section I need to mention one more result that follows (either directly, or by using 4.7) from the corresponding classical theorem in elementary calculus. (It will be used in the next section to find "definable points" on the zero sets of Pfaffian functions (cf. 2.7).) The easy details of the transfer (from $\overline{\overline{\mathbb{R}}}$ ) required for the proof are left to the reader.

4.10. Proposition. Suppose $n, s, g_{1}, \ldots, g_{s}$ are as in 4.8, $s<n$ and $P \in$ $V^{n s}\left(g_{1}, \ldots, g_{s}\right)$. Let $[g, W] \in \mathfrak{D}^{(n)}(P)$ and suppose that for some $U \in \mathfrak{G}_{P}$ (with $\left.U \subseteq W \cap \bigcap_{i=1}^{s} \operatorname{dom}\left(g_{i}\right)\right)$ we have $g(\vec{x}) \geq g(P)$ for all $\vec{x} \in U \cap V^{n s}\left(g_{1}, \ldots, g_{s}\right)$ (i.e. $P$ is a local minimum of $g$ on $\left.V^{n s}\left(g_{1}, \ldots, g_{s}\right)\right)$. Then the sequence of vectors $\left\langle d_{P} g_{1}, \ldots, d_{P} g_{s}, d_{P} g\right\rangle$ is linearly dependent.

\section{Definable points on Components And the Proof of Lemma 2.7}

I continue to use the notation of section 4 . In particular, $\overline{\bar{K}}$ denotes an arbitrary model of $\overline{\bar{T}}$.

Fix $n \in \mathbb{N}, n \geq 1$, and let $U$ be a definable open subset of $K^{n}$. Clearly $\{U\}$ is a n.s. in $K^{n}$ and we may safely identify both $\mathfrak{D}^{(n)}(\{U\})^{-}$and $\mathfrak{D}^{(n)}(\{U\})$ with the differential unital ring of all definable, infinitely differentiable functions from $U$ to $K$, which we denote by $\mathfrak{D}^{(n)}(U)$. If $P \in U$, then clearly the map $R_{P}: \mathfrak{D}^{(n)}(U) \rightarrow$ $\mathfrak{D}^{(n)}(P): f \mapsto[f, U]$ is a differential ring homomorphism which need be neither injective nor surjective in general. It is, however, clearly injective on the unital subring generated by the $n$ projection functions (restricted to $U$ ) and I use the usual notation, $\mathbb{Z}\left[x_{1}, \ldots, x_{n}\right]$, for this subring and for its $R_{P}$-image in $\mathfrak{D}^{(n)}(P)$.

5.1. Theorem. With the above notation let $M$ be a Noetherian subring of $\mathfrak{D}^{(n)}(U)$ which contains $\mathbb{Z}\left[x_{1}, \ldots, x_{n}\right]$ and which is closed under differentiation. Let $f \in M$ and suppose that $S$ is a non-empty definable subset of $V(f)$ which is both open in $V(f)$ (in the subspace topology) and closed in $K^{n}$. Then there exist $f_{1}, \ldots, f_{n} \in M$ such that $S \cap V^{n s}\left(f_{1}, \ldots, f_{n}\right) \neq \varnothing$.

Proof. For each $Q \in S$ let $I_{Q}$ be the ideal $\{g \in M: g(Q)=0\}$ of $M$. Since $M$ is Noetherian we may choose $P \in S$ such that $I_{P}$ is maximal in $\left\{I_{Q}: Q \in S\right\}$. Let $\left\{g_{1}, \ldots, g_{N}\right\}$ be a finite generating set for $I_{P}$ and set $g=\sum_{i=1}^{N} g_{i}^{2}$. Then $P \in V(g) \cap S$ and, further, we have

$$
I_{Q}=I_{P} \quad \text { for any } Q \in V(g) \cap S .
$$


Now choose $m$ maximal so that for some $f_{1}, \ldots, f_{m} \in M, P \in V^{n s}\left(f_{1}, \ldots, f_{m}\right)$. If $m=n$ we are done, so suppose, for a contradiction, that $m<n$ and fix such $f_{1}, \ldots, f_{m}$.

Claim 1. $V(g) \cap S \subseteq V^{n s}\left(f_{1}, \ldots, f_{m}\right)$.

Proof. Since $P \in V^{n s}\left(f_{1}, \ldots, f_{m}\right)$ we have $f_{1}, \ldots, f_{m} \in I_{P}$ and $\operatorname{det}(E) \notin I_{P}$ where $E$ is some $m \times m$ submatrix of the $m \times n$ matrix with rows $\left\langle\frac{\partial f_{i}}{\partial x_{1}}, \ldots, \frac{\partial f_{i}}{\partial x_{n}}\right\rangle$ for $1 \leq i \leq m$ (note that $\operatorname{det}(E) \in M$ since $M$ is closed under differentiation). Hence by $(*)$ we have that for any $Q \in V(g) \cap S, f_{1}, \ldots, f_{m} \in I_{Q}$ and $\operatorname{det}(E) \notin I_{Q}$ which immediately implies that $Q \in V^{n s}\left(f_{1}, \ldots, f_{m}\right)$, as required.

Claim 2. Let $Q \in V(g) \cap S$ and $h \in M$. Then $Q \notin V^{n s}\left(f_{1}, \ldots, f_{m}, h\right)$.

Proof. Suppose $Q \in V^{n s}\left(f_{1}, \ldots, f_{m}, h\right)$. Then arguing as in the proof of Claim 1 we would have $P \in V^{n s}\left(f_{1}, \ldots, f_{m}, h\right)$ which contradicts the maximality of $m$.

Claim 3. Let $Q \in V(g) \cap S$. Then there exists $W \in \mathfrak{G}_{Q}$ (with $W \subseteq U$ ) such that $W \cap V(g) \cap S=W \cap V^{n s}\left(f_{1}, \ldots, f_{m}\right)$.

Proof. Since $g \in I_{P}$ we have by $(*)$ that $g(Q)=0$. Hence, by Claim 2 and 4.9 (applied to the image of $M$ under the map $R_{Q}$ ), there exists $W^{\prime} \in \mathfrak{G}_{Q}$ (with $\left.W^{\prime} \subseteq U\right)$ such that $g$ vanishes on $W^{\prime} \cap V^{n s}\left(f_{1}, \ldots, f_{m}\right)$. It follows that every element of $I_{P}$, and in particular $f$, vanishes on $W^{\prime} \cap V^{n s}\left(f_{1}, \ldots, f_{m}\right)$. Thus $W^{\prime} \cap$ $V^{n s}\left(f_{1}, \ldots, f_{m}\right) \subseteq W^{\prime} \cap V(g) \cap V(f)$. But $S$ is open in $V(f)$ (by hypothesis) so for some $W^{\prime \prime} \in \mathfrak{G}_{Q}, W^{\prime \prime} \cap S=W^{\prime \prime} \cap V(f)$. Thus $W \cap V^{n s}\left(f_{1}, \ldots, f_{m}\right) \subseteq W \cap V(g) \cap S$ where $W=W^{\prime} \cap W^{\prime \prime}$. Claim 3 now follows from Claim 1.

Claim 4. $S \cap V(g)$ is closed in $K^{n}$.

Proof. This is immediate from the facts that $S$ is closed in $K^{n}$ (by hypothesis), $S \subseteq U$ and $g$ is (defined and) continuous on the open set $U$.

Now let $\vec{\eta}=\left\langle\eta_{1}, \ldots, \eta_{n}\right\rangle \in \mathbb{Z}^{n}$. By Claim 4 there is a point $Q \in S \cap V(g)$ whose distance from $\vec{\eta}$ is minimal (note also that $S \cap V(g) \neq \emptyset$ since $P \in S \cap V(g)$ ), i.e. $h(Q) \leq h(\vec{x})$ for all $\vec{x} \in S \cap V(g)$, where $h\left(x_{1}, \ldots, x_{n}\right)=\sum_{i=1}^{n}\left(x_{i}-\eta_{i}\right)^{2}$. Note that $h$ (restricted to $U$ ) is an element of $M$ since $\mathbb{Z}\left[x_{1}, \ldots, x_{n}\right] \subseteq M$. Further, by Claim 3, $Q$ is actually a local minimum of $h$ on $V^{n s}\left(f_{1}, \ldots, f_{m}\right)$, so by 4.10 the sequence of vectors $\left\langle d_{Q} f_{1}, \ldots, d_{Q} f_{m}, d_{Q} h\right\rangle$ is linearly dependent. Arguing as in the proof of Claim 1 it follows that $\left\langle d_{P} f_{1}, \ldots, d_{P} f_{m}, d_{P} h\right\rangle$ is linearly dependent. Since the sequence $\left\langle d_{P} f_{1}, \ldots, d_{P} f_{m}\right\rangle$ is linearly independent it follows that $d_{P} h$ lies in the subspace, call it $X$, of $K^{n}$ spanned (over $K$ ) by $d_{P} f_{1}, \ldots, d_{P} f_{m}$, for any $\vec{\eta} \in \mathbb{Z}$. Write $h=h_{\vec{\eta}}$. By an easy calculation, $\vec{\eta}=\frac{1}{2}\left(d_{P} h_{\overrightarrow{0}}-d_{P} h_{\vec{\eta}}\right)$. Hence $\mathbb{Z}^{n} \subseteq X$, which is impossible since $m<n$.

5.2. Proof of 2.7. We shall apply 5.1 with $\overline{\overline{\mathbb{R}}}=\widetilde{\mathbb{R}}, \overline{\bar{T}}=\widetilde{T}$ and $\widetilde{K}$ an arbitrary model of $\widetilde{T}$ (cf. the beginning of section 2). Let $n, r \in \mathbb{N}$ and suppose that $\vec{\sigma}$ is an $(n, r)$-sequence. Let $\tilde{k} \models \widetilde{T}, \tilde{k} \subseteq \widetilde{K}$, and set $U=D^{r}(\vec{\sigma}, \widetilde{K})$ so that $U$ is an open definable subset of $K^{r}$ (cf. 2.2). Further, by 2.3 and the comments between 2.4 and $2.5, M^{r}(\tilde{k}, \widetilde{K}, \vec{\sigma})$ is a subring of $\mathfrak{D}^{(r)}(U)$ which is closed under differentiation. It is also Noetherian, because it is finitely generated over the field $k$, and it clearly contains $\mathbb{Z}\left[x_{1}, \ldots, x_{r}\right]$ (in fact, $k\left[x_{1}, \ldots, x_{r}\right]$ ) as a subring.

Now to prove 2.7 , suppose $g \in M^{r}(\tilde{k}, \widetilde{K}, \vec{\sigma})$ and $g(P)=0$ for some $P \in U$. If we knew that $V(g)$ were closed in $K^{r}$, then we could apply 5.1 directly (with $n=r$, $M=M^{r}(\tilde{k}, \widetilde{K}, \vec{\sigma}), U=D^{r}(\vec{\sigma}, \widetilde{K}), f=g$ and $\left.S=V(g)\right)$ to obtain a $(\tilde{k}, \vec{\sigma})$-definable point $Q \in D^{r}(\vec{\sigma}, \widetilde{K})$ such that $Q \in V(g)$, thus completing the proof of 2.7 (with $s=0)$. Unfortunately, there is no reason to suppose that $V(g)$ does not have limit 
points on the boundary of $U$. However, this problem can be easily overcome by the standard geometric technique of pushing such points out to infinity. To do this we regard $\vec{\sigma}$ as an $(n, r+s)$-sequence, where $s=2 r$, in the sense of example $E$ of section 2. Now for $1 \leq i \leq r$ define

$$
\begin{gathered}
g_{i}\left(x_{1}, \ldots, x_{r+s}\right)= \begin{cases}x_{i} \cdot x_{r+i}-1 & \text { if } x_{i} \text { is } \vec{\sigma} \text {-bounded, } \\
x_{r+i}-x_{i} & \text { otherwise, }\end{cases} \\
g_{r+i}\left(x_{1}, \ldots, x_{r+s}\right)= \begin{cases}\left(x_{i}-1\right) x_{2 r+i}-1 & \text { if } x_{i} \text { is } \vec{\sigma} \text {-bounded, } \\
x_{2 r+i}-x_{i} & \text { otherwise. }\end{cases}
\end{gathered}
$$

Now set $f=g^{2}+\sum_{i=1}^{2 r} g_{i}^{2}$ and notice that if $\left\langle p_{1}, \ldots, p_{r}\right\rangle \in V(g)$, then $\left\langle p_{1}, \ldots, p_{r+s}\right\rangle$ $\in V(f)$ where $p_{r+i}=p_{2 r+i}=p_{i}$ if $x_{i}$ is not $\vec{\sigma}$-bounded, and $p_{r+i}=p_{i}^{-1}, p_{2 r+i}=$ $\left(p_{i}-1\right)^{-1}$ if $x_{i}$ is $\vec{\sigma}$-bounded (in which case we necessarily have that $0<p_{i}<1$ ). Thus $V(f) \neq \emptyset$ and it is easy to see that $V(f)$ is closed in $K^{r+s}$. We may therefore argue as above (this time using 5.1 with $n=r+s, M=M^{r+s}(\tilde{k}, \widetilde{K}, \vec{\sigma}), U=$ $D^{r+s}(\vec{\sigma}, \widetilde{K})=D^{r}(\vec{\sigma}, \widetilde{K}) \times K^{s}$, and $\left.S=V(f)\right)$ to obtain the conclusion of 2.7 .

Recall now Proposition 3.1. This states that 0-dimensional Pfaffian varieties are uniformly finite. Khovanskii has proved a natural generalization of this fact for arbitrary zero-sets of Pfaffian functions which turns out to follow from 3.1 and 5.1 using a simple model theoretic argument. Thus rather than simply quoting the result it seems worthwhile to include the proof here.

5.3. Theorem (Khovanskii). Suppose that $h_{1}, \ldots, h_{l}$ is any Pfaffian chain of functions on $\mathbb{R}^{m+n}$. Let $g \in \mathbb{R}\left[x_{1}, \ldots, x_{m+n}, h_{1}, \ldots, h_{l}\right]$. Then there is $N \in \mathbb{N}$ such that for any $Q \in \mathbb{R}^{n}$ the set $\left\{P \in \mathbb{R}^{m}: g(P, Q)=0\right\}$ has at most $N$ components.

(A component of a set $S \subseteq \mathbb{R}^{m}$ is a set $X \subseteq S$ such that $X$ is clopen in (the subspace) $S$. Clearly the collection of all components of $S$ forms a Boolean algebra.)

Proof. Suppose the theorem is false. Then for each $i \in \mathbb{N}$, there exist $Q^{(i)} \in \mathbb{R}^{n}$ and pairwise disjoint non-empty components, $C_{0}^{(i)}, \ldots, C_{i}^{(i)}$, of the set $\left\{P \in \mathbb{R}^{m}\right.$ : $\left.g\left(P, Q^{(i)}\right)=0\right\}$.

Let $\overline{\bar{L}}$ be any expansion of $\bar{L}$ that includes symbols for the functions $h_{1}, \ldots, h_{l}$, the set $\mathbb{N}$, the map $i \rightarrow Q^{(i)}(i \in \mathbb{N})$ and the $(m+2)$-ary relation " $P \in C_{j}^{(i)}$ ". Let $\overline{\overline{\mathbb{R}}}$ be the corresponding expansion of $\overline{\mathbb{R}}$ and suppose $\overline{\bar{K}}$ is a $\left(2^{\aleph_{0}}\right)^{+}$-saturated elementary extension of $\overline{\overline{\mathbb{R}}}$. Let $a$ be a nonstandard natural number in $K$. Then (the $\overline{\bar{K}}$ interpretations of) each $C_{i}^{(a)}$ (for $i \leq a, \overline{\bar{K}} \models$ " $i \in \mathbb{N}$ ") is a non-empty subset of $Z \stackrel{\text { def }}{=}\left\{P \in K^{m}: g\left(P, Q^{(a)}\right)=0\right\}$ which is both open and closed in $Z$, and hence also closed in $K^{m}$. Suppose $Q^{(a)}=\left\langle q_{1}, \ldots, q_{n}\right\rangle$ and let

$$
M=\mathbb{R}\left[x_{1}, \ldots, x_{m}, q_{1}, \ldots, q_{n}, h_{1}\left(x_{1}, \ldots, x_{m}, Q^{(a)}\right), \ldots, h_{l}\left(x_{1}, \ldots, x_{m}, Q^{(a)}\right)\right] .
$$

Then $M$ is a Noetherian ring of $\overline{\bar{K}}$-definable, infinitely differentiable functions on $K^{m}$ which contains $\mathbb{Z}\left[x_{1}, \ldots, x_{m}\right]$ and is closed under differentiation. Hence, by 5.1 , for each $i \leq a$ (with $\overline{\bar{K}} \models$ " $i \in \mathbb{N}$ ") there exist $f_{1}^{(i)}, \ldots, f_{m}^{(i)} \in M$ such that $C_{i}^{(a)} \cap V^{n s}\left(f_{1}^{(i)}, \ldots, f_{m}^{(i)}\right) \neq \emptyset$. But there are at most $2^{\aleph_{0}}$ possibilities for $f_{1}^{(i)}, \ldots, f_{m}^{(i)}$ and, by 3.1 , each $V^{n s}\left(f_{1}^{(i)}, \ldots, f_{m}^{(i)}\right)$ is finite. However, the collection $\left\{C_{i}^{(a)}: i \leq a\right.$, 
$\overline{\bar{K}} \models " i \in \mathbb{N} "\}$ consists of at least $\left(2^{\aleph_{0}}\right)^{+}$pairwise disjoint sets. This contradiction proves the theorem.

5.4. Corollary. Let $H_{1}, \ldots, H_{l}$ be a Pfaffian chain of functions on $\mathbb{R}^{m}$ ( $m \in$ $\mathbb{R}, m \geq 1$ ) and let $\widetilde{\mathbb{R}}^{\prime}$ be the structure $\left\langle\overline{\mathbb{R}} ; H_{1}, \ldots, H_{l} ; r\right\rangle_{r \in C}$ (where $C$ is any subset of $\mathbb{R})$ and $\widetilde{L}^{\prime}$ its language. Suppose that $\phi\left(x_{1}, x_{2}, \ldots, x_{p}\right)$ is an existential formula of $\widetilde{L}^{\prime}$. Then there exists $N \in \mathbb{N}$ such that for all $r_{2}, \ldots, r_{p} \in \mathbb{R}$ the set $\left\{r_{1} \in \mathbb{R}: \widetilde{\mathbb{R}}^{\prime} \models \phi\left(r_{1}, r_{2}, \ldots, r_{p}\right)\right\}$ is a union of at most $N$ open intervals and $N$ points.

Proof. By the usual tricks (cf. section 2 before Definition 2.1) we may suppose that $\phi\left(x_{1}, \ldots, x_{p}\right)$ has the form $\exists y_{1}, \ldots, y_{n} f\left(x_{1}, \ldots, x_{p}, y_{1}, \ldots, y_{n}\right)=0$, where $f$ is a term of $\widetilde{L}^{\prime}$. Now it is easy to construct a Pfaffian chain of functions on $\mathbb{R}^{p+n}$, $h_{1}, \ldots, h_{l^{\prime}}$ say, such that $f \in \mathbb{R}\left[\vec{x}, \vec{y}, h_{1}, \ldots, h_{l^{\prime}}\right]$. Thus by 5.3 there exists $N_{0} \in \mathbb{N}$ such that for all $r_{2}, \ldots, r_{p} \in \mathbb{R}$ the set $Z\left(r_{2}, \ldots, r_{p}\right) \stackrel{\text { def }}{=}\left\{\left\langle p, q_{1}, \ldots, q_{n}\right\rangle \in \mathbb{R}^{1+n}\right.$ : $\left.f\left(p, r_{2}, \ldots, r_{p}, q_{1}, \ldots, q_{n}\right)=0\right\}$ has at most $N_{0}$ components. But then clearly this is also true for $\pi\left[Z\left(r_{2}, \ldots, r_{p}\right)\right]$ where $\pi: \mathbb{R}^{1+n} \rightarrow \mathbb{R}$ is the projection map onto the first coordinate.

\section{One DIMENSIONAL VARIETIES}

In this section $\overline{\overline{\mathbb{R}}}$ denotes an expansion of $\overline{\mathbb{R}}$ which is either of the form $\widetilde{\mathbb{R}}$ (as described in section 1), or of the form $\widetilde{\mathbb{R}}^{\prime}$ as described in the hypothesis of Corollary 5.4. In the latter case the set $C$ of distinguished elements should be chosen to satisfy an analogous condition to the former case (cf. section 1, just after equation (2)). Clearly all the definitions from section 2 can be applied to the $\widetilde{\mathbb{R}}^{\prime}-\widetilde{L}^{\prime}-\widetilde{T}^{\prime}-\widetilde{K}^{\prime}$ $\widetilde{k}^{\prime}$ case and are, in fact, somewhat less complicated. For example, there is no need to allow $y_{1}, \ldots, y_{m}$ to be 0 or 1 in Definition 2.1 (i) (a), and $D^{r}\left(\vec{\sigma}, \widetilde{K}^{\prime}\right)$ is simply $K^{r}$ for any $(n, r)$-sequence $\vec{\sigma}$ and $\widetilde{K}^{\prime} \models \widetilde{T}^{\prime}$ (cf. Definition 2.2).

My aim in this section is to show that non-singular (space-) curves implicitly defined by terms in models of $\overline{\bar{T}}$ can be explicitly parameterized by finitely many infinitely differentiable definable functions having open intervals for domains. I first require the following combinatorial result.

6.1. Lemma. Let $n, N \in \mathbb{N}$ with $n, N \geq 1$. Then there exist $Q_{1}, \ldots, Q_{s} \in \mathbb{Z}^{n}$, where $s=n \cdot N^{2}+1$, with the property that for any field $K$ of characteristic 0 and any distinct elements $P_{1}, \ldots, P_{m} \in K^{n}$ (where $\left.m \leq N\right)$, there exists an $i, 1 \leq i \leq s$, such that $Q_{i} \cdot P_{1}, \ldots, Q_{i} \cdot P_{m}$ are distinct elements of $K$. (Here "." denotes the usual scalar product.)

Proof. Choose $Q_{1}, \ldots, Q_{s} \in \mathbb{Z}^{n}$ in general position, i.e. any $n$ of them are linearly independent over $\mathbb{Q}$ (and hence over any field of characteristic 0). Suppose, for a contradiction, that there exist $K, m(m \leq N)$ and distinct $P_{1}, \ldots, P_{m} \in K^{n}$ such that for each $i=1, \ldots, s, Q_{i} \cdot P_{\alpha_{i}}=Q_{i} \cdot \bar{P}_{\beta_{i}}$ for some $\alpha_{i}, \beta_{i}$ with $1 \leq \alpha_{i}<\beta_{i} \leq m$. Since the map $i \rightarrow\left\langle\alpha_{i}, \beta_{i}\right\rangle$ has domain of size $>n \cdot N^{2}$ and range of size $\leq N^{2}$, there exist $\alpha, \beta$ with $1 \leq \alpha<\beta \leq m$ such that $Q_{i} \cdot\left(P_{\alpha}-P_{\beta}\right)=0$ for $n$ distinct values of $i$. This contradicts the choice of the $Q_{i}$ 's since $P_{\alpha}-P_{\beta} \neq 0$. 
6.2. Theorem. Suppose $n, r \in \mathbb{N}, r \geq 2$, and that $\vec{\sigma}$ is an $(n, r)$-sequence. Let $\overline{\bar{k}}, \overline{\bar{K}} \models \overline{\bar{T}}$ with $\overline{\bar{k}} \subseteq \overline{\bar{K}}$ and suppose that $g_{1}, \ldots, g_{r-1} \in M^{r}(\overline{\bar{k}}, \overline{\bar{K}}, \vec{\sigma})$. Let $V=\{P \in$ $\left.D^{r}(\vec{\sigma}, \overline{\bar{K}}): g_{1}(P)=\cdots=g_{r-1}(P)=0\right\}$ and suppose that

(a) $V$ is a closed subset of $K^{r}$, and

(b) for all $P \in V$, $\operatorname{det}\left(\frac{\partial\left(g_{1}, \ldots, g_{r-1}\right)}{\partial\left(x_{2}, \ldots, x_{r}\right)}\right)(P) \neq 0$ (cf. the notation described before 2.5).

Then there exists a finite set $\mathcal{S}$ of pairs $\langle I, \phi\rangle$ such that

(i) for each $\langle I, \phi\rangle \in \mathcal{S}, I$ is an open interval in $K$ and $\phi: I \rightarrow K^{r-1}$ is an infinitely differentiable definable function;

(ii) for each $\langle I, \phi\rangle \in \mathcal{S}$, if $\sup I \in K$ (i.e. $\sup I \neq \infty)$, then $\|\phi(x)\| \rightarrow \infty$ as $x \rightarrow \sup I$ (from below), where $\|\cdot\|$ denotes the usual norm on $K^{r-1}$, and similarly for $\inf I$;

(iii) $V=\bigcup\{\operatorname{graph}(\phi):\langle I, \phi\rangle \in \mathcal{S}\}$ and the union is disjoint.

Proof. By 3.3 (or the analogous result in the case $\overline{\overline{\mathbb{R}}}=\widetilde{\mathbb{R}}^{\prime}$, which follows directly from 3.1) and (b) it follows that there is some $N \in \mathbb{N}$ such that for each $p_{1} \in K$ the set $V_{p_{1}}$ contains at most $N$ elements, where $V_{p_{1}}=\left\{\left\langle p_{2}, \ldots, p_{r}\right\rangle \in K^{r-1}\right.$ : $\left.\left\langle p_{1}, \ldots, p_{r}\right\rangle \in V\right\}$. Let $s=(r-1) \cdot N^{2}+1$ and let $Q_{1}, \ldots, Q_{s} \in \mathbb{Z}^{r-1}$ be as in Lemma 6.1 (with $n=r-1$ ). For each $m=1, \ldots, N$ and $i=1, \ldots, s$ set $A_{m, i}=\left\{p_{1} \in K: \operatorname{card}\left(V_{p_{1}}\right)=\operatorname{card}\left(Q_{i} \cdot V_{p_{1}}\right)=m\right\}$.

Now it is easy to see that each $A_{m, i}$ can be defined in $\overline{\bar{K}}$ by a boolean combination of existential formulas (with parameters) and hence, either by the comments following 3.5 (in the case $\overline{\overline{\mathbb{R}}}=\widetilde{\mathbb{R}}$ ) or by 5.4 (in the case $\overline{\overline{\mathbb{R}}}=\widetilde{\mathbb{R}}^{\prime}$ ), it is a finite union of open intervals and points. It clearly follows from this that there exist $t \in \mathbb{N}$ and $a_{1}, \ldots, a_{t} \in K$ such that (setting $\left.a_{0}=-\infty, a_{t+1}=+\infty\right)$ :

$$
\begin{aligned}
& a_{0}<a_{1}<\cdots<a_{t}<a_{t+1} \text { and for each } j=0, \ldots, t, \\
& i=1, \ldots, s, m=1, \ldots, N \text { and } p, q \in\left(a_{j}, a_{j+1}\right), \\
& p \in A_{m, i} \text { if and only if } q \in A_{m, i} .
\end{aligned}
$$

Now for $p \in K$ let $m(p)=\operatorname{card}\left(V_{p}\right)$ and $i(p)=$ the least $i$ such that $\operatorname{card}\left(Q_{i} \cdot V_{p}\right)=$ $m(p)$. Then $m(p) \leq N$ and $i(p)$ exists by the conclusion of 6.1 . Further, it clearly follows from $(*)$ that for each $j=0, \ldots, t$, if $a_{j}<p, q<a_{j+1}$, then $m(p)=m(q)$ and $i(p)=i(q)$ so we may denote these numbers by $m_{j}$ and $i_{j}$ respectively. Hence we may define functions $\phi_{j, l}:\left(a_{j}, a_{j+1}\right) \rightarrow K^{r-1}$ (for those $j=0, \ldots, t$ with $m_{j} \geq 1$ and $\left.l=1, \ldots, m_{j}\right)$ by

$$
\begin{aligned}
\phi_{j, l}(x)=\vec{y} \Leftrightarrow \exists \vec{y}^{(1)}, \ldots, \exists \vec{y}^{\left(m_{j}\right)}\left(\left\langle x, y^{(1)}\right\rangle \in V \wedge \cdots \wedge\left\langle x, \vec{y}^{\left(m_{j}\right)}\right\rangle \in V\right. \\
\left.\wedge Q_{i_{j}} \cdot \vec{y}^{(1)}<\cdots<Q_{i_{j}} \cdot \vec{y}^{\left(m_{j}\right)} \wedge \vec{y}=\vec{y}^{(l)}\right) .
\end{aligned}
$$

Now since the map $K^{r-1} \rightarrow K: \vec{y} \rightarrow Q_{i_{j}} \cdot \vec{y}$ is continuous it follows that each $\phi_{j, l}$ coincides locally with a function given by the implicit function theorem for $V$ (cf. the discussion at the beginning of section 4$)$ and hence is infinitely differentiable on $\left(a_{j}, a_{j+1}\right)$. We also clearly have that $\left\{\left\langle p_{1}, \ldots, p_{r}\right\rangle \in V: a_{j}<p_{1}<a_{j+1}\right\}=$ $\bigcup\left\{\operatorname{graph}\left(\phi_{j, l}\right): 1 \leq l \leq m_{j}\right\}$ where the union is disjoint.

Now suppose that $j<t\left(\right.$ so $a_{j+1} \neq \infty$ ) and $1 \leq l \leq m_{j}$. Then either $\left\|\phi_{j, l}(x)\right\| \rightarrow$ $\infty$ as $x \rightarrow a_{j+1}$ (from below) or else there is some $\left\langle p_{2}, \ldots, p_{r}\right\rangle \in K^{r-1}$ such that $\left\langle a_{j+1}, p_{2}, \ldots, p_{r}\right\rangle$ is a limit point of $\operatorname{graph}\left(\phi_{j, l}\right)$. For this is clear if $\overline{\bar{K}}=\overline{\overline{\mathbb{R}}}$, and since 
only the continuity of $\phi_{j, l}$ is required the result may be transferred to a general $\overline{\bar{K}}$. It follows from hypothesis (a) of the theorem that $\left\langle a_{j+1}, p_{2}, \ldots, p_{r}\right\rangle \in V$ (notice that the fact that $V$ is a closed subset of $D^{r}(\vec{\sigma}, \overline{\bar{K}})$ is not sufficient here) and hence, by (b) and the implicit function theorem, there is an open box neighbourhood, $U$ say, of $\left\langle p_{2}, \ldots, p_{r}\right\rangle$ in $K^{r-1}$, an $\varepsilon \in K$ with $a_{j}<a_{j+1}-\varepsilon<a_{j+1}<a_{j+1}+\varepsilon<a_{j+2}$, and a $K$-definable infinitely differentiable function $\phi:\left(a_{j+1}-\varepsilon, a_{j+1}+\varepsilon\right) \rightarrow U$ such that $\phi\left(a_{j+1}\right)=\left\langle p_{2}, \ldots, p_{r}\right\rangle$ and $V \cap\left(\left(a_{j+1}-\varepsilon, a_{j+1}+\varepsilon\right) \times U\right)=\operatorname{graph}(\phi)$. It must be the case that $\phi$ coincides with $\phi_{j, l}$ on $\left(a_{j+1}-\varepsilon, a_{j+1}\right)$ (because the set $\left\{p \in\left(a_{j+1}-\varepsilon, a_{j+1}\right): \phi(p)=\phi_{j, l}(p)\right\}$ is both open and closed in $\left(a_{j+1}-\varepsilon, a_{j+1}\right)$ and is non-empty since $\left(a_{j+1}-\varepsilon, a_{j+1}\right) \times U$ contains a point of $\operatorname{graph}\left(\phi_{j, l}\right)$ which is necessarily a point of $V$ and hence of $\operatorname{graph}(\phi))$ and, indeed, that there exists $l^{\prime}$ with $1 \leq l^{\prime} \leq m_{j+1}$ such that $\phi$ coincides with $\phi_{j+1, l^{\prime}}$ on $\left(a_{j+1}, a_{j+1}+\varepsilon\right)$. Thus $\phi_{j, l}, \phi_{j+1, l^{\prime}}$, and $\left\{\left\langle a_{j+1}, p_{2}, \ldots, p_{r}\right\rangle\right\}$ may be glued together to form a definable, infinitely differentiable function from $\left(a_{j}, a_{j+2}\right)$ to $K^{r-1}$ whose graph is contained in $V$. The theorem now follows by repeating this process until no further glueing across the $a_{j}$ 's is possible.

I shall refer to the set $\mathcal{S}$ given by 6.2 as a parameterization of $V$ in $\overline{\bar{K}}$. Of course, if $V \cap k^{r}$ is also closed in $k^{r}$, we may apply 6.2 with $\overline{\bar{K}}=\overline{\bar{k}}$ and obtain a parameterization, $\mathcal{S}^{\prime}$ say, of $V \cap k^{r}$ in $\overline{\bar{k}}$ but at the moment we cannot infer any relationship between $\mathcal{S}$ and $\mathcal{S}^{\prime}$. The following lemma clarifies the situation somewhat.

6.3. Lemma. Suppose that, in addition to the hypotheses of 6.2, every $(\overline{\bar{k}}, \vec{\sigma})$ definable point of $K^{r} \cap V$ (cf. 2.5) lies in $k^{r}$. Let $K^{-}=\{\alpha \in K:-\beta<\alpha<\beta$ for some $\beta \in k\}$ and suppose that $\alpha \in K^{-}, P \in K^{r-1},\|P\| \in K^{-}$and $\langle\alpha, P\rangle \in V$. Then there exist $\gamma_{1}, \gamma_{2}, \beta_{1}, \beta_{2}, B_{1}, B_{2} \in k$ with $\gamma_{2}<\gamma_{1}<\alpha<\beta_{1}<\beta_{2}$ and $\|P\|<$ $B_{1}<B_{2}, m \in \mathbb{N}(m \geq 1)$, and $\overline{\bar{K}}$-definable infinitely differentiable functions $\phi_{i}:\left(\gamma_{2}, \beta_{2}\right) \rightarrow K^{r-1}($ for $i=1, \ldots, m)$ such that

(i) $\left\|\phi_{i}(p)\right\|<B_{1}$ for $i=1, \ldots, m$ and $p \in\left(\gamma_{2}, \beta_{2}\right)$;

(ii) $V \cap\left(\left(\gamma_{2}, \beta_{2}\right) \times\left\{Q \in K^{r-1}:\|Q\|<B_{2}\right\}\right)=\bigcup_{i=1}^{m} \operatorname{graph}\left(\phi_{i}\right)$, and the union is disjoint.

Further, if $V \cap k^{r}$ is closed in $k^{r}$, there exist $\overline{\bar{k}}$-definable infinitely differentiable functions $\psi_{i}:\left(\gamma_{2}, \beta_{2}\right) \rightarrow k^{r-1}$ (for $\left.i=1, \ldots, m\right)$ such that (i) and (ii) hold with $\psi_{i}$ in place of $\phi_{i}$ where all notions are interpreted in $\overline{\bar{k}}$.

Remark. As I shall show below, it follows from the additional assumption on $\overline{\bar{k}}$ and $\overline{\bar{K}}$, and (b) of 6.2 , that if $1 \leq i \leq m, p \in k$ and $\gamma_{2}<p<\beta_{2}$, then $\phi_{i}(p) \in k^{r-1}$. However, there is still no guarantee that the function $\phi_{i} \uparrow k$ is equal to some $\psi_{i^{\prime}}$, or even that it is $\overline{\bar{k}}$-definable.

Proof. With the notation of the proof of 6.2 choose $m \in \mathbb{N}$ such that there are exactly $m$ points $Q \in V_{\alpha}$ such that $\|Q\| \in K^{-}$. Let $P_{1}, \ldots, P_{m}$ be these points and note that $m \geq 1$ since $P$ is one of them. Choose $B \in k$ such that $\left\|P_{i}\right\|<B$ for $i=1, \ldots, m$ and let $B^{\prime} \in k, B^{\prime}>B$. Then certainly $\|Q\|>B^{\prime}$ for all $Q \in V_{\alpha} \backslash\left\{P_{1}, \ldots, P_{m}\right\}$. For each $i=1, \ldots, m$ let $\left\langle I_{i}, \phi_{i}\right\rangle$ be the (unique) element of $\mathcal{S}$ such that $\alpha \in I_{i}$ and $\phi_{i}(\alpha)=P_{i}$. This is possible by (iii) of 6.2 . Now consider 
the $(\overline{\bar{K}}$-definable $)$ set $A^{+}\left(=A^{+}\left(B, B^{\prime}\right)\right)$ given by:

$$
\begin{array}{r}
A^{+}=\left\{p \in \bigcap_{i=1}^{m} I_{i}: p \geq \alpha \text { and for all } q \in[\alpha, p] \text { and } i=1, \ldots, m,\right. \\
\left\|\phi_{i}(q)\right\|<B, \text { and } \phi_{1}(q), \ldots, \phi_{m}(q) \text { are the only } \\
\text { points } \left.Q \in V_{q} \text { satisfying }\|Q\| \leq B^{\prime}\right\} .
\end{array}
$$

By (i), (ii) and (iii) of $6.2, A^{+}$has the form $[\alpha, \beta)$ where $\beta \in K \cup\{\infty\}$ and $\beta>\alpha$. If $\beta=\infty$, let $\beta_{1}, \beta_{2}$ be any elements of $k$ satisfying $\alpha<\beta_{1}<\beta_{2}$. This is possible since $\alpha \in K^{-}$. If $\beta \in K$, I claim that $\beta \in k$. For certainly $\beta \in \bigcap_{i=1}^{m} I_{i}$ since otherwise we clearly contradict (i) and (ii) of 6.2. It follows that there is some $Q \in V_{\beta}$ such that either $\|Q\|=B$ or $\|Q\|=B^{\prime}$. Define $g: D^{r}(\vec{\sigma}, \overline{\bar{K}}) \rightarrow K$ by $g\left(x_{1}, \ldots, x_{r}\right)=\sum_{i=2}^{r} x_{i}^{2}-B^{2}$ (in the former case) or $\sum_{i=2}^{r} x_{i}^{2}-\left(B^{\prime}\right)^{2}$ (in the latter case). Then $g \in M^{r}(\overline{\bar{k}}, \overline{\bar{K}}, \vec{\sigma})$ and $g$ vanishes at the point $\langle\beta, Q\rangle$ but does not vanish on $V \cap W$ for any open neighbourhood $W$ of $\langle\beta, Q\rangle$. It now follows from 4.9 (with $n=r, P_{0}=\langle\beta, Q\rangle, M=\left\{\left[f, D^{r}(\vec{\sigma}, \overline{\bar{K}})\right]: f \in M^{r}(\tilde{k}, \widetilde{K}, \vec{\sigma})\right\}$ and $\left.\left\{f_{1}, \ldots, f_{m}\right\}=\left\{g_{1}, \ldots, g_{r-1}\right\}\right)$ that $\langle\beta, Q\rangle$ is a $(\overline{\bar{k}}, \vec{\sigma})$-definable point of $K^{r} \cap V$ and hence lies in $k^{r}$. This proves the claim. Now let $\beta_{1}=\beta$ and choose $B_{1}, B_{2} \in k$ such that $B<B_{1}<B_{2}<B^{\prime}$. Then $A^{+}\left(B_{1}, B_{2}\right)=\left[\alpha, \beta^{\prime}\right)$ for some $\beta^{\prime} \in k \cup\{\infty\}$ and clearly $\beta^{\prime}>\beta_{1}$. If $\beta^{\prime} \in k$ set $\beta_{2}=\beta^{\prime}$. If $\beta^{\prime}=\infty$ set $\beta_{2}=\beta_{1}+1$. By the definition of $A^{+}$we now have that (i) and (ii) hold with $\alpha$ in place of $\gamma_{2}$. However, the elements $\gamma_{1}, \gamma_{2}$ of $k$ can be obtained by a similar argument by considering $A^{-}$ (where " $p \geq \alpha$ ", " $[\alpha, p]$ " are replaced by " $p \leq \alpha$ ", " $[p, \alpha]$ " in the definition of $A^{+}$) with the same $B, B^{\prime}, B_{1}, B_{2}$.

To establish the last part of the lemma first observe that the result mentioned in the remark follows from 4.9 since if $\langle p, Q\rangle \in V$ and $p \in k$, then the function $D^{r}(\vec{\sigma}, \overline{\bar{K}}) \rightarrow K:\left\langle x_{1}, \ldots, x_{r}\right\rangle \rightarrow x_{1}-p$ is in $M^{r}(\overline{\bar{k}}, \overline{\bar{K}}, \vec{\sigma})$, vanishes at $\langle p, Q\rangle$, but certainly does not vanish locally on $V$ at $\langle p, Q\rangle$. Hence, since $V$ has a quantifier-free definition (with parameters in $k$ ), it follows from (i) and (ii) that for each $p \in k$ with $\gamma_{2}<p<\beta_{2}$ there are exactly $m$ points $Q \in k^{r-1}$ such that $\overline{\bar{k}}=(\langle p, Q\rangle \in$ $V \wedge\|Q\|<B_{2}$ ), and each such point satisfies $\|Q\|<B_{1}$. Let $Q_{1}, \ldots, Q_{m}$ be these points for the choice $p=\frac{\gamma_{2}+\beta_{2}}{2}$. Now let $\mathcal{S}^{\prime}$ be a parameterization of $V$ in $\overline{\bar{k}}$ and for $i=1, \ldots, m$ choose (the unique) $\left\langle I_{i}^{\prime}, \psi_{i}\right\rangle \in \mathcal{S}^{\prime}$ such that $\psi_{i}\left(\frac{\gamma_{2}+\beta_{2}}{2}\right)=Q_{i}$. Then since each map $x \mapsto\left\|\psi_{i}(x)\right\|$ is continuous on $\left(\gamma_{2}, \beta_{2}\right) \cap I_{i}^{\prime}$, it follows from the intermediate value theorem (interpreted in $\overline{\bar{k}}$ ) that it takes no value $\geq B_{1}$. In particular (by (ii) of 6.2) $\left(\gamma_{2}, \beta_{2}\right) \subseteq I_{i}^{\prime}$. This proves the lemma.

\section{The PROOF OF Lemma 2.8}

I shall in fact prove 2.8 for both the $\widetilde{T}$ and $\widetilde{T}^{\prime}$ situation, so let $\overline{\overline{\mathbb{R}}}, \overline{\bar{T}}$ etc. be as described at the beginning of section 6 . The proof is by induction on $n$. The base step is provided by example (D) of section 2. For the induction step suppose $n, r \in \mathbb{N}, \overline{\bar{K}} \models \overline{\bar{T}}, \overline{\bar{k}} \models \overline{\bar{T}}, \overline{\bar{k}} \subseteq \overline{\bar{K}}$ and that $\left\langle\vec{\sigma}, \sigma_{n+1}\right\rangle$ is an $(n+1, r)$-sequence (where $\vec{\sigma}$ is an $(n, r)$-sequence) such that

$$
\text { for all } s \geq r \text {, every }\left(\overline{\bar{k}},\left\langle\vec{\sigma}, \sigma_{n+1}\right\rangle\right) \text {-definable }
$$$$
\text { point of } K^{s} \text { lies in }\left(K^{-}\right)^{s} \text {. }
$$ 
(Here, $K^{-}$is as defined in the statement of Lemma 6.3.)

Now suppose $s \geq r$. Since every $\vec{\sigma}$-bounded variable (cf. 2.1 (ii)) is also $\left\langle\vec{\sigma}, \sigma_{n+1}\right\rangle$ bounded we have that $D^{s}\left(\left\langle\vec{\sigma}, \sigma_{n+1}\right\rangle, \overline{\bar{K}}\right) \subseteq D^{s}(\vec{\sigma}, \overline{\bar{K}})$. Further, if $g \in M^{s}(\overline{\bar{k}}, \overline{\bar{K}}, \vec{\sigma})$, then $g \uparrow D^{s}\left(\left\langle\vec{\sigma}, \sigma_{n+1}\right\rangle, \overline{\bar{K}}\right) \in M^{s}\left(\overline{\bar{k}}, \overline{\bar{K}},\left\langle\vec{\sigma}, \sigma_{n+1}\right\rangle\right)$ and there will be no harm in identifying $M^{s}(\overline{\bar{k}}, \overline{\bar{K}}, \vec{\sigma})$ with its image in $M^{s}\left(\overline{\bar{k}}, \overline{\bar{K}},\left\langle\vec{\sigma}, \sigma_{n+1}\right\rangle\right)$ under this restriction mapping. (Similar remarks apply with $\overline{\bar{K}}$ replaced everywhere by $\overline{\bar{k}}$.) Clearly our inductive hypothesis and (8) imply

$$
\begin{aligned}
& \text { for all } s \geq r \text { and } P \in K^{s}, \text { if } P \text { is } \\
& (\overline{\bar{k}}, \vec{\sigma}) \text {-definable and } P \in D^{s}\left(\left\langle\vec{\sigma}, \sigma_{n+1}\right\rangle, \overline{\bar{K}}\right), \text { then } P \in k^{s} .
\end{aligned}
$$

Now let $Q$ be any $\left(\overline{\bar{k}},\left\langle\vec{\sigma}, \sigma_{n+1}\right\rangle\right)$-definable point of $K^{r}$. We must show that $Q \in k^{r}$.

Now, by definition, there exist $g_{1}, \ldots, g_{r} \in M^{r}\left(\overline{\bar{k}}, \overline{\bar{K}},\left\langle\vec{\sigma}, \sigma_{n+1}\right\rangle\right)$ such that

$$
g_{1}(Q)=\cdots=g_{r}(Q)=0,
$$

and

$$
\operatorname{det}\left(\frac{\partial\left(g_{1}, \ldots, g_{r}\right)}{\partial\left(x_{1}, \ldots, x_{r}\right)}\right)(Q) \neq 0
$$

Further,

$$
Q \in D^{r}\left(\left\langle\vec{\sigma}, \sigma_{n+1}\right\rangle, \overline{\bar{K}}\right) .
$$

I shall now deduce that $Q \in k^{r}$ under several extra assumptions on the sequence of functions $g_{1}, \ldots, g_{r}$. These will be justified later. For the moment, set $V=\{P \in$ $\left.K^{r}: g_{1}(P)=\cdots=g_{r-1}(P)=0\right\}$ (we may clearly suppose that $r \geq 2$ ), and assume that

$$
g_{1}, \ldots, g_{r-1} \in M^{r}(\overline{\bar{k}}, \overline{\bar{K}}, \vec{\sigma})
$$

$V$ is a closed subset of $K^{r}$ and $V \cap k^{r}$ is a closed subset of $k^{r}$;

$$
\begin{gathered}
V \subseteq D^{r}\left(\left\langle\vec{\sigma}, \sigma_{n+1}\right\rangle, \overline{\bar{K}}\right) \\
\operatorname{det}\left(\frac{\partial\left(g_{1}, \ldots, g_{r-1}\right)}{\partial\left(x_{2}, \ldots, x_{r}\right)}\right)(P) \neq 0 \text { for all } P \in V \\
\text { for all } P \in V \text {, if } g_{r}(P)=0 \text { then } \operatorname{det}\left(\frac{\partial\left(g_{1}, \ldots, g_{r}\right)}{\partial\left(x_{1}, \ldots, x_{r}\right)}\right)(P)<0 .
\end{gathered}
$$

Now notice that all the hypotheses of 6.3 are satisfied. (The fact that every $(k, \vec{\sigma})$-definable point of $K^{r} \cap V$ lies in $k^{r}$ follows from (15) and (9).) Further, since $Q$ is $\left(\overline{\bar{k}},\left\langle\vec{\sigma}, \sigma_{n+1}\right\rangle\right)$-definable, we have $Q \in\left(K^{-}\right)^{r}$ by (8). Hence we may apply 6.3 with $\alpha=q_{1}$ and $P=\left\langle q_{2}, \ldots, q_{r}\right\rangle$ (where $Q=\left\langle q_{1}, \ldots, q_{r}\right\rangle$ ) to obtain $\gamma_{1}, \gamma_{2}, \beta_{1}, \beta_{2}, B_{1}, B_{2} \in k, \phi_{i}:\left(\gamma_{2}, \beta_{2}\right) \rightarrow K^{r-1}$ and $\psi_{i}:\left(\gamma_{2}, \beta_{2}\right) \cap k \rightarrow k^{r-1}$ (for $i=1, \ldots, m)$ satisfying the conclusions of that lemma.

Now let $\phi$ be any one of the $\phi_{i}$ 's. Notice that for $t \in\left(\gamma_{2}, \beta_{2}\right)$ we have $\langle t, \phi(t)\rangle \in V$ and hence $($ by $(15))\langle t, \phi(t)\rangle \in D^{r}\left(\left\langle\vec{\sigma}, \sigma_{n+1}\right\rangle, \overline{\bar{K}}\right)$. Therefore we may define, for any 
$g \in M^{r}\left(\overline{\bar{k}}, \overline{\bar{K}},\left\langle\vec{\sigma}, \sigma_{n+1}\right\rangle\right)$, a function $\bar{g}:\left(\gamma_{2}, \beta_{2}\right) \rightarrow K$ by $\bar{g}(t)=g(t, \phi(t))$. Clearly $\bar{g}$ is $\overline{\bar{K}}$-definable and infinitely differentiable. Its first derivative is given by

$$
\frac{d \bar{g}}{d t}(t)=\frac{\overline{\partial g}}{\partial x_{1}}(t)+\sum_{i=2}^{r} \frac{\overline{\partial g}}{\partial x_{i}}(t) \cdot \frac{d \phi^{(i)}}{d t}(t)
$$

where $\phi(t)=\left\langle\phi^{(2)}(t), \ldots, \phi^{(r)}(t)\right\rangle$. Of course this formula holds for $\bar{g}=\bar{g}_{1}, \ldots, \bar{g}_{r-1}$, which are identically zero, and I leave the reader to perform the linear algebra required to eliminate the $\frac{d \phi^{(i)}}{d t}(t)$ terms and arrive at

$$
\frac{d \bar{g}}{d t}(t)=(-1)^{r+1} \bar{J}(t) \cdot \bar{J}_{1}(t)^{-1} \quad \text { for all } t \in\left(\gamma_{2}, \beta_{2}\right)
$$

where

$$
J\left(x_{1}, \ldots, x_{r}\right)=\operatorname{det}\left(\frac{\partial\left(g_{1}, \ldots, g_{r-1}, g\right)}{\partial\left(x_{1}, \ldots, x_{r}\right)}\right)
$$

and

$$
J_{1}\left(x_{1}, \ldots, x_{r}\right)=\operatorname{det}\left(\frac{\partial\left(g_{1}, \ldots, g_{r-1}\right)}{\partial\left(x_{2}, \ldots, x_{r}\right)}\right) .
$$

(Notice that (18) makes sense since $J, J_{1} \in M^{r}\left(\overline{\bar{k}}, \overline{\bar{K}},\left\langle\vec{\sigma}, \sigma_{n+1}\right\rangle\right)$ and, by (16), $\bar{J}_{1}(t) \neq 0$ for all $t \in\left(\gamma_{2}, \beta_{2}\right)$.)

I shall now assume that $r$ is even and leave the reader to make the obvious modifications to the argument for the case of $r$ odd.

7.1. Claim. (i) If $p \in\left(\gamma_{2}, \beta_{2}\right)$ and $\bar{g}_{r}(p)=0$, then $\frac{d \bar{g}_{r}}{d t}(p)$ has the same sign as $\bar{J}_{1}(p)$.

(ii) $\bar{g}_{r}$ has at most one zero.

Proof. (i) By (17) $\bar{J}(p)<0$, where $J$ is defined as in (18) with $g=g_{r}$. Thus (i) now follows immediately from (18) and the fact that $r$ is even.

(ii) Note that, by $(16), \bar{J}_{1}$ is non-zero throughout $\left(\gamma_{2}, \beta_{2}\right)$ and hence has constant sign on $\left(\gamma_{2}, \beta_{2}\right)$. It follows from (i) that $\frac{d \bar{g}_{r}}{d t}\left(p_{1}\right)$ and $\frac{d \bar{g}_{r}}{d t}\left(p_{2}\right)$ have the same (nonzero) sign whenever $\bar{g}_{r}\left(p_{1}\right)=\bar{g}_{r}\left(p_{2}\right)=0$. This is impossible (by transfer from $\overline{\mathbb{R}}$ ) unless $\bar{g}_{r}$ has at most one zero.

Now notice that (13)-(17) all hold with $\overline{\bar{k}}$ in place of $\overline{\bar{K}}$ and $V \cap k^{r}$ in place of $V$. This is because each of these statements actually implies the corresponding statement for $\overline{\bar{k}}$ and $V \cap k^{r}$. Hence the discussion above holds good in $\overline{\bar{k}}$ if we take $\phi$ to be one of the $\psi_{i}$ 's.

Now for any $g \in M^{r}\left(\overline{\bar{k}}, \overline{\bar{K}},\left\langle\sigma, \sigma_{n+1}\right\rangle\right)$, let $\bar{g}\left(\phi_{i} ; \cdot\right)$ be the $(\overline{\bar{K}}$-definable $)$ function from $\left\{t \in K: \gamma_{2}<t<\beta_{2}\right\}$ to $K$ obtained as above with $\phi=\phi_{i}$ and let $\bar{g}\left(\psi_{i} ; \cdot\right)$ be the ( $\bar{k}$-definable) function from $\left\{t \in k: \gamma_{2}<t<\beta_{2}\right\}$ to $k$ obtained with $\phi=\psi_{i}$ (note that $\gamma_{2}, \beta_{2} \in k$ ). We complete the proof of 2.8 (under the extra assumptions) as follows.

Let $i_{0}$ be the (unique) number such that $1 \leq i_{0} \leq m$ and $\phi_{i_{0}}\left(q_{1}\right)=\left\langle q_{2}, \ldots, q_{r}\right\rangle$. Suppose that $\bar{J}_{1}\left(\phi_{i_{0}} ; q_{1}\right)>0$. (The proof is similar if $\bar{J}_{1}\left(\phi_{i_{0}} ; q_{1}\right)<0$.) Let $S=$ $\left\{i: 1 \leq i \leq m\right.$ and $\left.\bar{J}_{1}\left(\phi_{i} ; q_{1}\right)>0\right\}$. Then, just as in the proof of 7.1 , it follows from (16) that $\bar{J}_{1}\left(\phi_{i} ; t\right)>0$ for all $i \in S$ and all $t \in\left(\gamma_{2}, \beta_{2}\right)$ and that $\bar{J}_{1}\left(\phi_{i} ; t\right)<0$ for all $i \in\{1, \ldots, m\} \backslash S$ and all $t \in\left(\gamma_{2}, \beta_{2}\right)$. In particular $\bar{J}_{1}\left(\phi_{i} ; \gamma_{1}\right)>0$ for $i \in S$ and $\bar{J}_{1}\left(\phi_{i} ; \gamma_{1}\right)<0$ for $i \in\{1, \ldots, m\} \backslash S$. It now follows from 6.3 (and the remark there) 
that there is a subset, $S^{\prime}$ say, of $\{1, \ldots, m\}$ such that $\left\{\psi_{i}\left(\gamma_{1}\right): i \in S^{\prime}\right\}=\left\{\phi_{i}\left(\gamma_{1}\right)\right.$ : $i \in S\}$, and hence that $\bar{J}_{1}\left(\psi_{i} ; t\right)>0$ (respectively $<0$ ) for all $i \in S^{\prime}$ (respectively $\left.i \in\{1, \ldots, m\} \backslash S^{\prime}\right)$ and $t \in\left(\gamma_{2}, \beta_{2}\right) \cap k$. Now $\overline{\bar{k}}$ is a substructure of $\overline{\bar{K}}$ so we clearly have (again using 6.3) that for all $t \in\left(\gamma_{2}, \beta_{2}\right) \cap k,\left\{\psi_{i}(t): i \in S^{\prime}\right\}=\left\{\phi_{i}(t): i \in S\right\}$. Now choose $\gamma_{3}, \beta_{3} \in k$ such that $\gamma_{2}<\gamma_{3}<\gamma_{1}$ and $\beta_{1}<\beta_{3}<\beta_{2}$ and such that for no $i=1, \ldots, m$ does either $\bar{g}_{r}\left(\phi_{i} ; \cdot\right)$ or $\bar{g}_{r}\left(\psi_{i} ; \cdot\right)$ have a zero at $\gamma_{3}$ or $\beta_{3}$. This is possible since there are at most a finite number of points to be avoided. By 7.1 (and its version for $\overline{\bar{k}}$ ) it clearly follows that if $i \in S$ (respectively $i \in S^{\prime}$ ), then $\bar{g}_{r}\left(\phi_{i} ; \cdot\right)$ has a zero in $\left(\gamma_{3}, \beta_{3}\right)$ (respectively, $\bar{g}_{r}\left(\psi_{i} ; \cdot\right)$ has one in $\left.\left(\gamma_{3}, \beta_{3}\right) \cap k\right)$ if and only if $\bar{g}_{r}\left(\phi_{i} ; \gamma_{3}\right)<0$ and $\bar{g}_{r}\left(\phi_{i} ; \beta_{3}\right)>0$ (respectively $\bar{g}_{r}\left(\psi_{i} ; \gamma_{3}\right)<0$ and $\bar{g}_{r}\left(\psi_{i} ; \beta_{3}\right)>0$ ). Hence

$$
\begin{aligned}
& \operatorname{card}\left\{i \in S: \exists t \in\left(\gamma_{3}, \beta_{3}\right) \bar{g}_{r}\left(\phi_{i} ; t\right)=0\right\} \\
& \quad=\operatorname{card}\left\{i \in S: \bar{g}_{r}\left(\phi_{i} ; \gamma_{3}\right)<0\right\}-\operatorname{card}\left\{i \in S: \bar{g}_{r}\left(\phi_{i} ; \beta_{3}\right)<0\right\}
\end{aligned}
$$

and

$$
\begin{aligned}
& \operatorname{card}\left\{i \in S^{\prime}: \exists t \in\left(\gamma_{3}, \beta_{3}\right) \cap k \bar{g}_{r}\left(\psi_{i} ; t\right)=0\right\} \\
& \quad=\operatorname{card}\left\{i \in S^{\prime}: \bar{g}_{r}\left(\psi_{i} ; \gamma_{3}\right)<0\right\}-\operatorname{card}\left\{i \in S^{\prime}: \bar{g}_{r}\left(\psi_{i} ; \beta_{3}\right)<0\right\} .
\end{aligned}
$$

However, by 6.3 (and the fact that $\overline{\bar{k}} \subseteq \overline{\bar{K}}$ ) the two right hand sides here are equal. It now follows (again using 6.3) that every point $P=\left\langle p_{1}, \ldots, p_{r}\right\rangle \in K^{r}$ satisfying $P \in V, g_{r}(P)=0, J_{1}(P)>0, \gamma_{3}<p_{1}<\beta_{3}$ and $\left\|\left\langle p_{2}, \ldots, p_{r}\right\rangle\right\|<B_{1}$ actually lies in $k^{r}$. But $Q$ is such a point!

I must now show why (13)-(17) may be assumed. So suppose that $g_{1}, \ldots, g_{r}$ and $Q$ satisfy (10)-(12). I shall modify $\left\langle\vec{\sigma}, \sigma_{n+1}\right\rangle$ (to $\left.\left\langle\vec{\sigma}^{\prime}, \sigma_{n+1}^{\prime}\right\rangle\right)$ so that (8) and (9) are still satisfied, and produce $h_{1}, \ldots, h_{s} \in M^{s}\left(\overline{\bar{k}}, \overline{\bar{K}},\left\langle\vec{\sigma}^{\prime}, \sigma_{n+1}^{\prime}\right\rangle\right)$ for some $\left.s \geq r\right)$ and a point $Q^{\prime} \in K^{s}$ such that (10)-(17) are satisfied with $h_{1}, \ldots, h_{s}, Q^{\prime}$ in place of $g_{1}, \ldots, g_{r}, Q$. Further, $q_{1}, \ldots, q_{r}$ will occur amongst the coordinates of $Q^{\prime}$. This is clearly sufficient. The new functions and point will be produced in several stages but to avoid a proliferation of notation I shall revert to the original notation (i.e. $\left.g_{1}, \ldots, g_{r}, Q\right)$ at the end of the justification of each stage. The conditions (10)-(12) will be satisfied at each stage.

Stage 1. We may assume that for each $\left\langle\vec{\sigma}, \sigma_{n+1}\right\rangle$-bounded variable $x$, there are variables $y, z$ such that both $x \cdot y^{2}-1$ and $(1-x) \cdot z^{2}-1$ occur amongst $g_{1}, \ldots, g_{r}$.

Justification. Suppose that $x_{i}$ is $\left\langle\vec{\sigma}, \sigma_{n+1}\right\rangle$-bounded (where $1 \leq i \leq r$ ). Define $g_{r+1}, g_{r+2} \in M^{r+2}\left(\overline{\bar{k}}, \overline{\bar{K}},\left\langle\vec{\sigma}, \sigma_{n+1}\right\rangle\right)$ by $g_{r+1}\left(x_{1}, \ldots, x_{r+1}\right)=x_{i} \cdot x_{r+1}^{2}-1$, $g_{r+2}\left(x_{1}, \ldots, x_{r+2}\right)=\left(1-x_{i}\right) \cdot x_{r+2}^{2}-1$. Then, since $0<q_{i}<1$ (because of (12)), we may set $q_{r+1}=+q_{i}^{-\frac{1}{2}}$ and $q_{r+2}=+\left(1-q_{i}\right)^{-\frac{1}{2}}$ so that (10) and (12) are clearly satisfied for $g_{1}, \ldots, g_{r+2},\left\langle Q, q_{r+1}, q_{r+2}\right\rangle$. Further, as a simple calculation shows,

$\operatorname{det}\left(\frac{\partial\left(g_{1}, \ldots, g_{r+2}\right)}{\partial\left(x_{1}, \ldots, x_{r+2}\right)}\right)\left(Q, q_{r+1}, q_{r+2}\right)=\operatorname{det}\left(\frac{\partial\left(g_{1}, \ldots, g_{r}\right)}{\partial\left(x_{1}, \ldots, x_{r}\right)}\right)(Q) \cdot 4 \cdot q_{i}^{\frac{1}{2}} \cdot\left(1-q_{i}\right)^{\frac{1}{2}}$

and the right hand side is non-zero by (11) (for $\left.g_{1}, \ldots, g_{r}, Q\right)$. Hence (11) holds for the new system.

Stage 2. We may assume that $g_{1}, \ldots, g_{r-1} \in M^{r}(\overline{\bar{k}}, \overline{\bar{K}}, \vec{\sigma})$ and that $g_{r}$ has the form $\sigma_{n+1}\left(x_{1}, \ldots, x_{r}\right)-x_{e}$, where $x_{e}$ is not $\left\langle\vec{\sigma}, \sigma_{n+1}\right\rangle$-bounded (and hence does not actually occur in the term $\left.\sigma_{n+1}\left(x_{1}, \ldots, x_{r}\right)\right)$. 
Justification. By definition of $M^{r}\left(\overline{\bar{k}}, \overline{\bar{K}},\left\langle\vec{\sigma}, \sigma_{n+1}\right\rangle\right)$ there exist $h_{1}, \ldots, h_{r} \in$ $M^{r}(\overline{\bar{k}}, \overline{\bar{K}}, \vec{\sigma})\left[x_{r+1}\right]\left(=M^{r+1}(\overline{\bar{k}}, \overline{\bar{K}}, \vec{\sigma})\right)$ such that

$$
g_{i}\left(x_{1}, \ldots, x_{r}\right)=h_{i}\left(x_{1}, \ldots, x_{r}, \sigma_{n+1}\left(x_{1}, \ldots, x_{r}\right)\right)
$$

for $i=1, \ldots, r$. Let $q_{r+1}=\sigma_{n+1}\left(q_{1}, \ldots, q_{r}\right), Q^{\prime}=\left\langle Q, q_{r+1}\right\rangle$ and $h_{r+1}\left(x_{1}, \ldots, x_{r+1}\right)$ $=\sigma_{n+1}\left(x_{1}, \ldots, x_{r}\right)-x_{r+1}$. Clearly (10) and (12) are satisfied for $h_{1}, \ldots, h_{r+1}, Q^{\prime}$ as well as stage 1 and stage 2 . For (11), consider the matrix $\frac{\partial\left(h_{1}, \ldots, h_{r+1}\right)}{\partial\left(x_{1}, \ldots, x_{r+1}\right)}\left(Q^{\prime}\right)$. For each $i=1, \ldots, r$, multiply row $r+1$ by $\frac{\partial h_{i}}{\partial x_{r+1}}\left(Q^{\prime}\right)$ and add the result to row $i$. By the chain rule, the resulting matrix has determinant $-\operatorname{det}\left(\frac{\partial\left(g_{1}, \ldots, g_{r}\right)}{\partial\left(x_{1}, \ldots, x_{r}\right)}\right)(Q)$ which is non-zero by (the old) (11).

Stage 3. We may assume that for all $P \in D^{r}\left(\left\langle\vec{\sigma}, \sigma_{n+1}\right\rangle, \overline{\bar{K}}\right)$, if $g_{i}(P)=0$ for $i=1, \ldots, r-1$, then $\operatorname{det}\left(\frac{\partial\left(g_{1}, \ldots, g_{r}-1\right)}{\partial\left(x_{2}, \ldots, x_{r}\right)}\right)(P) \neq 0$.

Justification. By (11) there is some $i(1 \leq i \leq r)$ such that

$$
\operatorname{det}\left(\frac{\partial\left(g_{1}, \ldots, g_{r-1}\right)}{\partial\left(x_{1}, \ldots, x_{i-1}, x_{i+1}, \ldots, x_{r}\right)}\right)(Q) \neq 0 .
$$

By relabelling the variables we may suppose that $i=1$. (Note that for every $n, r \in \mathbb{N}$, the notion of an $(n, r)$-sequence is invariant under permutation of variables. Further, the definable points for the permuted sequence are just coordinate permutations of definable points for the original sequence. Thus (8) and (9) are still true for the permuted sequence. Clearly so are (10)-(12) and stages 1 and 2 for the corresponding transformation of $g_{1}, \ldots, g_{r}$ and $Q$.)

Now let

$$
h\left(x_{1}, \ldots, x_{r+1}\right)=x_{r+1} \cdot \operatorname{det}\left(\frac{\partial\left(g_{1}, \ldots, g_{r-1}\right)}{\partial\left(x_{2}, \ldots, x_{r}\right)}\right)\left(x_{1}, \ldots, x_{r}\right)-1,
$$

and set

$$
q_{r+1}=\operatorname{det}\left(\frac{\partial\left(g_{1}, \ldots, g_{r-1}\right)}{\partial\left(x_{2}, \ldots, x_{r}\right)}\right)(Q)^{-1}, \quad Q^{\prime}=\left\langle Q, q_{r+1}\right\rangle .
$$

Then $g_{1}, \ldots, g_{r-1}, h, g_{r}$ and $Q^{\prime}$ still satisfy stages 1 and 2 and also, clearly, (10) and (12). For (11), a simple calculation shows that

$$
\operatorname{det}\left(\frac{\partial\left(g_{1}, \ldots, g_{r-1}, h, g_{r}\right)}{\partial\left(x_{1}, \ldots, x_{r+1}\right)}\right)\left(Q^{\prime}\right)=-\operatorname{det}\left(\frac{\partial\left(g_{1}, \ldots, g_{r}\right)}{\partial\left(x_{1}, \ldots, x_{r}\right)}\right)(Q) \cdot q_{r+1}^{-1}
$$

which is non-zero by (the old) (11).

Finally, to see that stage 3 is satisfied suppose that $P \in D^{r+1}\left(\left\langle\vec{\sigma}, \sigma_{n+1}\right\rangle, \overline{\bar{K}}\right)$ and that $g_{1}(P)=\cdots=g_{r-1}(P)=h(P)=0$. Say $P=\left\langle p_{1}, \ldots, p_{r+1}\right\rangle$. Since $h(P)=0$ we have $p_{r+1} \neq 0$ and routine calculation gives $\operatorname{det}\left(\frac{\partial\left(g_{1}, \ldots, g_{r-1}, h\right)}{\partial\left(x_{2}, \ldots, x_{r+1}\right)}\right)(P)=p_{r+1}^{-2} \neq 0$, as required.

Stage 4. We may assume that for all $P \in D^{r}\left(\left\langle\vec{\sigma}, \sigma_{n+1}\right\rangle, \overline{\bar{K}}\right)$, if $g_{i}(P)=0$ for $i=1, \ldots, r$, then $\operatorname{det}\left(\frac{\partial\left(g_{1}, \ldots, g_{r}\right)}{\partial\left(x_{1}, \ldots, x_{r}\right)}\right)(P)<0$.

Justification. As in the proof of stage 2 , there is some $h \in M^{r}(\overline{\bar{k}}, \overline{\bar{K}}, \vec{\sigma})[z]$ such that

$$
\operatorname{det}\left(\frac{\partial\left(g_{1}, \ldots, g_{r}\right)}{\partial\left(x_{1}, \ldots, x_{r}\right)}\right)\left(x_{1}, \ldots, x_{r}\right)=h\left(x_{1}, \ldots, x_{r}, \sigma_{n+1}\left(x_{1}, \ldots, x_{r}\right)\right) .
$$


Define $H \in M^{r+1}(\overline{\bar{k}}, \overline{\bar{K}}, \vec{\sigma})$ by $H\left(x_{1}, \ldots, x_{r+1}\right)=x_{r+1} \cdot h\left(x_{1}, \ldots, x_{r}, x_{e}\right)-1$ where $e$ is as given by stage 2 (so $1 \leq e \leq r$ ). Now since $g_{r}\left(q_{1}, \ldots, q_{r}\right)=0$, i.e. $\sigma_{n+1}\left(q_{1}, \ldots, q_{r}\right)=q_{e}$, it follows from $(*)$ that $h\left(q_{1}, \ldots, q_{r}, q_{e}\right)=\operatorname{det}\left(\frac{\partial\left(g_{1}, \ldots, g_{r}\right)}{\partial\left(x_{2}, \ldots, x_{r}\right)}\right)(Q)$ which is non-zero by (11). Hence we may set $q_{r+1}=h\left(q_{1}, \ldots, q_{r}, q_{e}\right)^{-1}$ and $Q^{\prime}=\left\langle Q, q_{r+1}\right\rangle$ so that $(10)$, (12), stages 1 and 2 are clearly satisfied for the system $g_{1}, \ldots, g_{r-1}, H, g_{r}, Q^{\prime}$. To see that stage 4 (and hence (11)) are also satisfied, suppose that $\left\langle p_{1}, \ldots, p_{r+1}\right\rangle=P \in D^{r+1}\left(\left\langle\vec{\sigma}, \sigma_{n+1}\right\rangle, \overline{\bar{K}}\right)$ and that $g_{1}(P)=\cdots=$ $g_{r-1}(P)=H(P)=g_{r}(P)=0$. Then by routine calculation we obtain

$$
\begin{aligned}
\operatorname{det}( & \left.\frac{\partial\left(g_{1}, \ldots, g_{r-1}, H, g_{r}\right)}{\partial\left(x_{1}, \ldots, x_{r+1}\right)}\right)(P)=-\operatorname{det}\left(\frac{\partial\left(g_{1}, \ldots, g_{r-1}, g_{r}, H\right)}{\partial\left(x_{1}, \ldots, x_{r+1}\right)}\right)(P) \\
& =-\operatorname{det}\left(\frac{\partial\left(g_{1}, \ldots, g_{r}\right)}{\partial\left(x_{1}, \ldots, x_{r}\right)}\right)\left(p_{1}, \ldots, p_{r}\right) \cdot h\left(p_{1}, \ldots, p_{r}, p_{e}\right) \\
& =-h\left(p_{1}, \ldots, p_{r}, p_{e}\right)^{2}
\end{aligned}
$$

(by $(*)$ and the fact that $\left.g_{r}\left(p_{1}, \ldots, p_{r}\right)=0\right)$. Since $H(P)=0, h\left(p_{1}, \ldots, p_{r}, p_{e}\right) \neq 0$ so the conclusion of stage 4 follows. Finally, stage 3 is still satisfied because if $P=\left\langle p_{1}, \ldots, p_{r+1}\right\rangle$ is any point in $D^{r+1}\left(\left\langle\vec{\sigma}, \sigma_{n+1}\right\rangle, \overline{\bar{K}}\right)$ such that $g_{1}(P)=\cdots=$ $g_{r-1}(P)=H(P)=0$, then

$$
\begin{aligned}
\operatorname{det}( & \left.\frac{\partial\left(g_{1}, \ldots, g_{r-1}, H\right)}{\partial\left(x_{2}, \ldots, x_{r+1}\right)}\right)(P) \\
\quad & =\operatorname{det}\left(\frac{\partial\left(g_{1}, \ldots, g_{r-1}\right)}{\partial\left(x_{2}, \ldots, x_{r}\right)}\right)\left(p_{1}, \ldots, p_{r}\right) \cdot h\left(p_{1}, \ldots, p_{r}, p_{e}\right)
\end{aligned}
$$

which is non-zero by (the old) stage 3 and the fact that $H(P)=0$.

The proof of Lemma 2.8 is now complete, for (13) follows from stage 2, (14) and (15) from stage $1,(16)$ from stage 3 and (17) from stage 4. Further, (8)-(12) were preserved throughout.

I shall prove Lemma 2.9 for the $\widetilde{T}$ situation (and hence complete the proof of the first main theorem) in the next section. I conclude this section, however, with the best result I know for the unrestricted $\left(\widetilde{T}^{\prime}\right)$ case. The proof follows immediately from 2.8 (for the $\widetilde{T}^{\prime}$ situation) and 2.7 (the proof of which-given in 5.2-clearly also works (in fact, more smoothly) for the $\widetilde{T}^{\prime}$-situation).

7.2. Theorem. Let $H_{1}, \ldots, H_{l}$ be a Pfaffian chain of functions on $\mathbb{R}^{m}$ ( $m \in$ $\mathbb{N}, m \geq 1)$ and let $\widetilde{\mathbb{R}}^{\prime}$ be the structure $\left\langle\overline{\mathbb{R}} ; H_{1}, \ldots, H_{l} ; r\right\rangle_{r \in C}$ where the set $C$ is chosen as at the beginning of section 6 . Let $\tilde{k}^{\prime}, \widetilde{K}^{\prime} \models \widetilde{T}^{\prime}, \tilde{k}^{\prime} \subseteq \widetilde{K}^{\prime}$, and suppose that for all $n, r \in \mathbb{N}$ and all $(n, r)$-sequences $\vec{\sigma}$, every $\left(\tilde{k}^{\prime}, \vec{\sigma}\right)$-definable point $\left\langle p_{1}, \ldots, p_{r}\right\rangle$ of $\left(K^{\prime}\right)^{r}$ satisfies $-B<p_{i}<B(i=1, \ldots, r)$ for some $B \in k^{\prime}$. (In particular, this is satisfied if $\widetilde{K}^{\prime}$ is a cofinal extension of $\tilde{k}^{\prime}$.) Then for any existential formula $\phi\left(x_{1}, \ldots, x_{e}\right)$ of $\widetilde{L}^{\prime}$, and any $a_{1}, \ldots, a_{e} \in k^{\prime}$ we have $\tilde{k}^{\prime} \models \phi\left(a_{1}, \ldots, a_{e}\right)$ if and only if $\widetilde{K}^{\prime} \models \phi\left(a_{1}, \ldots, a_{e}\right)$.

\section{The PROOF OF LemMa 2.9}

In this section I revert to the $\widetilde{T}$-situation of section 1 . The proof of 2.9 that I shall give here does not work for the $\widetilde{T}^{\prime}$-situation because it relies heavily on 3.5 and 
I know of no analogue of this result for this situation. (I say "analogue" because 3.5 as it stands obviously fails for, e.g., $\widetilde{T}^{\prime}=$ Theory $(\langle\overline{\mathbb{R}}, \exp \rangle)$ with exp unrestricted.)

So suppose $\widetilde{K}, \tilde{k} \models \widetilde{T}, \tilde{k} \subseteq \widetilde{K}$ and $n, r \in \mathbb{N}$. Let $\vec{\sigma}=\left\langle\sigma_{1}, \ldots, \sigma_{n}\right\rangle$ be an $(n, r)$ sequence and suppose that for each $s \geq r$ every $(\tilde{k}, \vec{\sigma})$-definable point of $K^{s}$ lies in $k^{s}$. Suppose $\sigma_{n+1}$ is such that $\left\langle\vec{\sigma}, \sigma_{n+1}\right\rangle$ is an $(n+1, r)$-sequence. It is clearly sufficient to show that every $\left(\tilde{k},\left\langle\vec{\sigma}, \sigma_{n+1}\right\rangle\right)$-definable point of $K^{r}$ lies in $\left(K^{-}\right)^{r}$ where, as before, $K^{-}=\{\alpha \in K:-\beta<\alpha<\beta$ for some $\beta \in k\}$.

Let $Q=\left\langle q_{1}, \ldots, q_{r}\right\rangle$ be a $\left(\tilde{k},\left\langle\vec{\sigma}, \sigma_{n+1}\right\rangle\right)$-definable point of $K^{r}$. Then, by applying the stages described in the previous section, we may assume that ( $r \geq 2$ and) there are functions $g_{1}, \ldots, g_{r} \in M^{r}\left(\tilde{k}, \widetilde{K},\left\langle\vec{\sigma}, \sigma_{n+1}\right\rangle\right)$ such that:

$$
g_{1}, \ldots, g_{r-1} \in M^{r}(\tilde{k}, \widetilde{K}, \vec{\sigma}) \text {; }
$$

$g_{r}$ has the form $\sigma_{n+1}\left(x_{1}, \ldots, x_{r}\right)-x_{e}$, where $x_{e}$ is not $\left\langle\vec{\sigma}, \sigma_{n+1}\right\rangle$-bounded;

$$
g_{i}(Q)=0 \text { for } i=1, \ldots, r \text { and } \operatorname{det}\left(\frac{\partial\left(g_{1}, \ldots, g_{r}\right)}{\partial\left(x_{1}, \ldots, x_{r}\right)}\right)(Q) \neq 0
$$

and, setting $V=\left\{P \in D^{r}(\vec{\sigma}, \widetilde{K}): g_{i}(P)=0\right.$ for $\left.i=1, \ldots, r-1\right\}$ :

$$
\left.V \subseteq D^{r}\left(\left\langle\vec{\sigma}, \sigma_{n+1}\right\rangle, \widetilde{K}\right) \text { and } V \text { (respectively } V \cap k^{r}\right)
$$
is a closed subset of $K^{r}$ (respectively $k^{r}$ );

$$
\text { for all } P \in V, \quad \operatorname{det}\left(\frac{\partial\left(g_{1}, \ldots, g_{r-1}\right)}{\partial\left(x_{2}, \ldots, x_{r}\right)}\right)(P) \neq 0 \text {; }
$$

$$
\text { for all } P \in V \text {, if } g_{r}(P)=0 \text { then } \operatorname{det}\left(\frac{\partial\left(g_{1}, \ldots, g_{r}\right)}{\partial\left(x_{1}, \ldots, x_{r}\right)}\right)(P) \neq 0 .
$$

The hypothesis of 2.9 may now be strengthened as follows.

8.1. Claim. Suppose $\chi\left(x_{1}, \ldots, x_{r}\right)$ is a formula of $\bar{L}$ (the language of ordered rings) possibly containing parameters from $k$. Suppose further that for some $\left\langle p_{1}, \ldots, p_{r}\right\rangle \in V, \widetilde{K} \models \chi\left(p_{1}, \ldots, p_{r}\right)$. Then for some $\left\langle p_{1}, \ldots, p_{r}\right\rangle \in V \cap k^{r}$, $\tilde{k} \models \chi\left(p_{1}, \ldots, p_{r}\right)$.

Proof. By quantifier elimination and the usual tricks we may suppose that $\chi\left(x_{1}, \ldots, x_{r}\right)$ has the form $\exists x_{r+1}, \ldots, \exists x_{r+t} \rho\left(x_{1}, \ldots, x_{r+t}\right)=0$ where $\rho$ is a polynomial with coefficients in $k$. Let $g=\rho^{2}+\sum_{i=1}^{r-1} g_{i}^{2}$. Then $g \in M^{r+t}(\tilde{k}, \widetilde{K}, \vec{\sigma})$ (by (19)) and $g(P)=0$ for some $P \in D^{r+t}(\vec{\sigma}, \widetilde{K})$. Hence by 2.7 there is some $\left\langle P, P^{\prime}\right\rangle \in$ $D^{(r+t)+s}(\vec{\sigma}, \widetilde{K})$ (for some $s \in \mathbb{N}$ ) such that $g(P)=0$ and $\left\langle P, P^{\prime}\right\rangle$ is $(\tilde{k}, \vec{\sigma})$-definable. By the hypothesis of $2.9,\left\langle P, P^{\prime}\right\rangle \in k^{(r+t)+s}$. Clearly if $P=\left\langle p_{1}, \ldots, p_{r+t}\right\rangle$, then $\left\langle p_{1}, \ldots, p_{r}\right\rangle$ satisfies the conclusion of the claim.

I now suppose, for a contradiction, that $Q \notin\left(I^{-}\right)^{r}$.

8.2. Claim. $q_{1} \notin k$. 
Proof. Suppose $q_{1} \in k$. Let $h\left(x_{1}, \ldots, x_{r}\right)=x_{1}-q_{1}$. Then $h \in M^{r}(\tilde{k}, \widetilde{K}, \vec{\sigma})$, $h(Q)=g_{1}(Q)=\cdots=g_{r-1}(Q)=0$ and

$$
\operatorname{det}\left(\frac{\partial\left(h, g_{1}, \ldots, g_{r-1}\right)}{\partial\left(x_{1}, \ldots, x_{r}\right)}\right)(Q)=\operatorname{det}\left(\frac{\partial\left(g_{1}, \ldots, g_{r-1}\right)}{\partial\left(x_{2}, \ldots, x_{r}\right)}\right)(Q) \neq 0
$$

(by (23)). Thus $Q$ is a $(\tilde{k}, \vec{\sigma})$-definable point of $K^{r}$ and so lies in $k^{r}\left(\subseteq\left(K^{-}\right)^{r}\right)$-a contradiction.

Now by (19), (22), (23) and 6.2 (see also the comments following the proof of 6.2) there exists a parameterization, $\left\{\left\langle I_{j}, \psi_{j}\right\rangle: 1 \leq j \leq N\right\}$ say, of $V \cap k^{r}$ in $\tilde{k}$. (Note, by the way, that $V \cap k^{r} \neq \emptyset$ by 8.1.) Let $I_{j}=\left(a_{j}, b_{j}\right)$ where $a_{j} \in k \cup(-\infty\}$, $b_{j} \in k \cup\{+\infty\}$ for $j=1, \ldots, N$.

8.3. Claim. Either $q_{1} \notin K^{-}$, or else there is some $j=1, \ldots, N$ such that either $0<q_{1}-a_{j}<\alpha$ or $0<b_{j}-q_{1}<\alpha$ for all $\alpha \in k$ with $\alpha>0$.

Proof. Suppose $q_{1} \in K^{-}$. Now we must have $a_{j}<q_{1}<b_{j}$ for some $j=1, \ldots, N$, for otherwise we could find $a, b \in k$ with $a<q_{1}<b$ such that no $\left\langle p_{1}, \ldots, p_{r}\right\rangle \in V \cap k^{r}$ satisfies the formula $a<x_{1}<b$, and this contradicts 8.1. Let $a=\max \left\{a_{j}: 1 \leq\right.$ $j \leq N$ and $\left.a_{j}<q_{1}<b_{j}\right\}$ and $b=\min \left\{b_{j}: 1 \leq j \leq N\right.$ and $\left.a_{j}<q_{1}<b_{j}\right\}$. Suppose, for a contradiction, that there is some $\alpha \in k, \alpha>0$ such that $q_{1}-a>\alpha$ and $b-q_{1}>\alpha$. Then $a<a+\alpha<q_{1}<b-\alpha<b$ so clearly $[a+\alpha, b-\alpha] \subseteq I_{j}$ for all $j$ such that $a_{j}<q_{1}<b_{j}$. (In the case $a=-\infty$, replace $a+\alpha$ by any element of $k$ which is less than $q_{1}$. This is possible since $q_{1} \in K^{-}$. Proceed similarly if $b=\infty$.)

Now since each $\psi_{j}$ is continuous, there is some $B \in k$ such that $\left\|\psi_{j}(t)\right\|<B$ for all $j$ such that $a_{j}<q_{1}<b_{j}$ and for all $t \in k$ with $a+\alpha \leq t \leq b-\alpha$. Now let $c=\max \left(\{a+\alpha\} \cup\left\{b_{j}: b_{j}<q_{1}\right\}\right)$ and $d=\min \left(\{b-\alpha\} \cup\left\{a_{j}: a_{j}>q_{1}\right\}\right)$. Then by 8.2 (and 6.2), there is no $\left\langle p_{1}, \ldots, p_{r}\right\rangle \in V \cap k^{r}$ with $c<p_{1}<d$ and $\left\|\left\langle p_{2}, \ldots, p_{r}\right\rangle\right\| \geq B$. This contradicts 8.1 since $Q$ is such a point in $V$.

I now claim that in addition to (19)-(24) we may assume that:

$$
q_{1}>\alpha \text { for all } \alpha \in k .
$$

For if this is not already the case, then by 8.3 we have (for some $a, b \in k$ ) either (a) $q_{1}<\alpha$ for all $\alpha \in k$, or (b) $0<q_{1}-a<\alpha$ for all $\alpha \in k, \alpha>0$, or (c) $0<b-q_{1}<\alpha$ for all $\alpha \in k, \alpha>0$. Define $h \in M^{r+1}(\tilde{k}, \widetilde{K}, \vec{\sigma})$ by

$$
h\left(x_{1}, \ldots, x_{r+1}\right)= \begin{cases}x_{1}+x_{r+1} & \text { in case (a), } \\ x_{r+1}\left(x_{1}-a\right)-1 & \text { in case (b), } \\ x_{r+1}\left(b-x_{1}\right)-1 & \text { in case (c) } .\end{cases}
$$

In all cases there is a unique $q_{r+1} \in K$ such that $\left\langle Q, q_{r+1}\right\rangle$ (= $Q^{\prime}$, say) satisfies $g_{1}\left(Q^{\prime}\right)=\cdots=g_{r-1}\left(Q^{\prime}\right)=h\left(Q^{\prime}\right)=g_{r}\left(Q^{\prime}\right)=0$, and clearly $q_{r+1}>\alpha$ for all $\alpha \in k$. Further, by immediate inspection or routine calculation, (19)-(22) and (24) all hold for the system $g_{1}, \ldots, g_{r-1}, h, g_{r}, Q^{\prime}$. Actually, (23) holds too, but more relevant for present purposes is the fact (again proved by direct calculation) that if $P \in K^{r+1}$ and $g_{1}(P)=\cdots=g_{r-1}(P)=h(P)=0$, then $\operatorname{det}\left(\frac{\partial\left(g_{1}, \ldots, g_{r-1}, h\right)}{\partial\left(x_{1}, \ldots, x_{r}\right)}\right)(P) \neq 0$. Now relabel variables (as in the justification of stage (3) in section 7 ) so that $x_{r+1}$ becomes $x_{1}$. Then (19)-(25) are satisfied for the new system, and we revert to the original notation. 
8.4. Claim. There exists a finite subset $S$ of $k$, an element $B$ of $k$ and a positive rational number $\theta$ such that:

(i) $0 \leq \alpha \leq 1$ for all $\alpha \in S$;

(ii) for any $\left\langle p_{1}, \ldots, p_{r}\right\rangle \in K^{r}$ with $p_{1}>B$ and $\left\langle p_{1}, \ldots, p_{r}\right\rangle \in V$, and any $i$ such that the variable $x_{i}$ is $\left\langle\vec{\sigma}, \sigma_{n+1}\right\rangle$-bounded, there exists $a \in S$ such that $\left|p_{i}-a\right|<p_{1}^{-\theta}$.

Proof. By 8.1 it is sufficient to prove this claim with $K$ replaced by $k$ in (ii), so we work in $\tilde{k}$. Let $\mathcal{S}$ be a parameterization of $V \cap k^{r}$ in $\tilde{k}$ and suppose $\langle I, \psi\rangle \in \mathcal{S}$ is such that $I$ is unbounded to the right. Say $\psi=\left\langle\psi_{2}, \ldots, \psi_{r}\right\rangle$. Suppose that the variable $x_{i}$ is $\left\langle\vec{\sigma}, \sigma_{n+1}\right\rangle$-bounded. Then by $(22)$ and $(25), 2 \leq i \leq r$ and $0<\psi_{i}(t)<1$ for all $t \in I$. It clearly follows from 3.5 (with $\widetilde{K}=\tilde{k}, g=\psi_{i}$ ) that $\psi_{i}(t) \rightarrow a_{i}$ as $t \rightarrow \infty$ for some $a_{i} \in k$ with $0 \leq a_{i} \leq 1$. Further, by applying 3.5 again with $g=\psi_{i}-a_{i}$, there exists a positive rational, $\theta_{i}$ say, such that $\left|\psi_{i}(t)-a_{i}\right|<t^{-\theta_{i}}$ for all sufficiently large $t \in k$. The claim now follows since there are only finitely many possibilities for $\langle I, \psi\rangle$ and $i$.

8.5. Claim. There exists a positive integer $\mu$ and an element $B^{\prime}$ of $k$ such that for any $\left\langle p_{1}, \ldots, p_{r}\right\rangle \in V \cap k^{r}$ with $p_{1}>B^{\prime}$ we have $\left|g_{r}\left(p_{1}, \ldots, p_{r}\right)\right|>p_{1}^{-\mu}$.

Proof. By (24) and 3.3, $g_{r}$ has only finitely many zeros on $V \cap k^{r}$. The claim now follows from 3.5 by an argument similar to that of 8.4. (Consider $g(t)=$ $\left.g_{r}\left(t, \psi_{2}(t), \ldots, \psi_{r}(t)\right).\right)$

Of course we would be done if we could show that 8.5 remained true with $V$ in place of $V \cap k^{r}$. To achieve this we shall approximate $g_{r}$ by a polynomial (uniformly in both $\widetilde{K}$ and $\tilde{k}$ ) and apply 8.1.

By $(20), g_{r}\left(x_{1}, \ldots, x_{r}\right)$ has the form $\sigma_{n+1}\left(x_{1}, \ldots, x_{r}\right)-x_{e}$, and by 2.1 , $\sigma_{n+1}\left(x_{1}, \ldots, x_{r}\right)$ has the form $F_{i}\left(y_{1}, \ldots, y_{m}\right)$ for some $i=1, \ldots, l$ and some $y_{1}, \ldots$, $y_{m} \in\left\{0,1, x_{1}, \ldots, x_{r}\right\}$. Now (working in $\mathbb{R}$ ) consider the function $G_{i}: U \rightarrow \mathbb{R}$ (cf. section 1). Recall that $U$ is an open set containing $[0,1]^{m}, G_{i}$ is $C^{\infty}$ (in fact analytic) on $U$ and $G_{i} \uparrow[0,1]^{m}=F_{i} \uparrow[0,1]^{m}$. From now on I write $F, G$ for $F_{i}, G_{i}$ respectively.

Since $[0,1]^{m}$ is compact, there exists a positive rational number $\varepsilon$ such that for each $P \in[0,1]^{m}, B_{\varepsilon}(P)\left(\stackrel{\text { def }}{=}\right.$ the open Euclidean ball in $\mathbb{R}^{m}$ with centre $P$ and radius $\varepsilon$ ) is contained in $U$. We may further assume that $G$ and all its derivatives are bounded (though not necessarily uniformly) on $\bigcup\left\{B_{\varepsilon}(P): P \in[0,1]^{m}\right\}$. Now by Taylor's theorem with Lagrange's form of the remainder, we have

$$
G\left(p_{1}+t_{1}, \ldots, p_{m}+t_{m}\right)=\sum_{i=0}^{\lambda}\left[\frac{1}{i !}\left(\sum_{j=1}^{m} t_{j} \frac{\partial}{\partial x_{j}}\right)^{i} G\right](P)+R_{\lambda},
$$

for all $P=\left\langle p_{1}, \ldots, p_{m}\right\rangle \in[0,1]^{m},\left\langle t_{1}, \ldots, t_{m}\right\rangle \in B_{\varepsilon}(0)$ and $\lambda \in \mathbb{N}$, where

$$
R_{\lambda}=\left[\frac{1}{(\lambda+1) !}\left(\sum_{j=1}^{m} t_{j} \frac{\partial}{\partial x_{j}}\right)^{\lambda+1} G\right]\left(P^{\prime}\right)
$$

for some $P^{\prime} \in B_{\varepsilon}(P)$. 
By our boundedness assumption on $G$ it follows that for all $\lambda \in \mathbb{N}$, there exists $C_{\lambda} \in \mathbb{N}$ such that for all $\left\langle t_{1}, \ldots, t_{m}\right\rangle \in B_{\varepsilon}(0)$,

$$
\left.\left|R_{\lambda}\right|<C_{\lambda} \cdot\left(\max \left\{\left|t_{i}\right|: 1 \leq i \leq m\right)\right\}\right)^{\lambda+1} .
$$

Now by (1), (2) (see the beginning of section 1) and (26), (28) it follows that for all $\lambda \in \mathbb{N}$ and all monomials $\pi\left(x_{1}, \ldots, x_{m}\right)$ of degree $\leq \lambda$, there exist terms $\tau_{\pi}^{\lambda}\left(x_{1}, \ldots, x_{m}\right)$ of $\widetilde{L}$ such that for all $P=\left\langle p_{1}, \ldots, p_{m}\right\rangle \in[0,1]^{m}$ and $\left\langle t_{1}, \ldots, t_{m}\right\rangle \in$ $B_{\varepsilon}(0)$ with $\left\langle p_{1}+t_{1}, \ldots, p_{m}+t_{m}\right\rangle \in B_{\varepsilon}(P) \cap[0,1]^{m}$, we have

$$
\begin{aligned}
& \left|\lambda ! F\left(p_{1}+t_{1}, \ldots, p_{m}+t_{m}\right)-\sum_{\pi}^{(\lambda)} \tau_{\pi}^{\lambda}(P) \cdot \pi\left(t_{1}, \ldots, t_{m}\right)\right| \\
& <\lambda ! C_{\lambda}\left(\max \left\{\left|t_{i}\right|: 1 \leq i<m\right\}\right)^{\lambda+1}
\end{aligned}
$$

where the summation is over the monomials of degree $\leq \lambda$.

I now want to apply $(29)$ in $\widetilde{K}$ (and $\tilde{k}$ ). Recall that $\sigma_{n+1}\left(x_{1}, \ldots, x_{r}\right)$ has the form $F\left(y_{1}, \ldots, y_{m}\right)$ for some $y_{1}, \ldots, y_{m} \in\left\{0,1, x_{1}, \ldots, x_{r}\right\}$. I therefore define, for each $\left\langle p_{1}, \ldots, p_{r}\right\rangle \in K^{r}$ and $i=1, \ldots, m$,

$$
p_{i}^{\prime}= \begin{cases}0 & \text { if } y_{i}=0 \\ 1 & \text { if } y_{i}=1 \\ p_{j} & \text { if } y_{i}=x_{j}\end{cases}
$$

Thus, if $\left\langle p_{1}, \ldots, p_{r}\right\rangle \in D^{r}\left(\left\langle\vec{\sigma}, \sigma_{n+1}\right\rangle, \widetilde{K}\right)$ (in particular, if $\left\langle p_{1}, \ldots, p_{r}\right\rangle \in V$-see $(22))$, then $0 \leq p_{i}^{\prime} \leq 1$ for $i=1, \ldots, m$ and $\sigma_{n+1}\left(p_{1}, \ldots, p_{r}\right)=F\left(p_{1}^{\prime}, \ldots, p_{m}^{\prime}\right)$. $\frac{\mu+1}{\theta}$.

Now let $S, \theta, B$ be as in $8.4, \mu, B^{\prime}$ as in 8.5 , and let $\lambda_{0}$ be an integer greater than

Consider the point $Q \in K^{r}$. Then $Q \in V$ and $q_{1}>B$ (by (25)) so we may define $a_{i}$ (for $\left.i=1, \ldots, m\right)$ as the unique $a \in S \cup\{0,1\}$ such that $\left|q_{i}^{\prime}-a\right|<q_{1}^{-\theta}$. Note that $a_{i} \in k$ and $0 \leq a_{i} \leq 1$ for $i=1, \ldots, m$. Further, $\left\langle q_{1}^{\prime}-a_{1}, \ldots, q_{m}^{\prime}-a_{m}\right\rangle \in B_{\varepsilon}(0)$ (since $0 \leq q_{1}^{-\theta}<\varepsilon, \varepsilon, \theta$ being positive rationals) and $\left\langle q_{1}^{\prime}, \ldots, q_{m}^{\prime}\right\rangle \in B_{\varepsilon}\left(\left\langle a_{1}, \ldots, a_{m}\right\rangle\right) \cap$ $[0,1]^{m}$. Also, $g_{r}(Q)=0$ so $F\left(q_{1}^{\prime}, \ldots, q_{m}^{\prime}\right)=q_{e}$. Hence, by $(29)$ applied in $\widetilde{K}$, we obtain:

$$
\left|\lambda_{0} ! q_{e}-\sum_{\pi}^{\left(\lambda_{0}\right)} \tau_{\pi}^{\lambda_{0}}\left(a_{1}, \ldots, a_{m}\right) \cdot \pi\left(q_{1}^{\prime}-a_{1}, \ldots, q_{m}^{\prime}-a_{m}\right)\right|<\lambda_{0} ! C_{\lambda_{0}} \cdot q_{1}^{-\theta\left(\lambda_{0}+1\right)}
$$

We also clearly have

$$
q_{1}>\max \left(B^{\prime}, 2 C_{\lambda}, \varepsilon^{-\theta^{-1}}\right)
$$

and

$$
\left|q_{i}^{\prime}-a_{i}\right|<q_{1}^{-\theta}, \quad \text { for } i=1, \ldots, m \text {. }
$$

Now since $\tilde{k} \subseteq \widetilde{K}$, all the $\tau_{\pi}^{\lambda_{0}}\left(a_{1}, \ldots, a_{m}\right)$ 's are elements of $k$ (and the evaluation of the term is absolute between $\widetilde{K}$ and $\tilde{k}$ ). We may therefore express the conjunction of $(30),(31)$ and $(32)$ as $\chi\left(q_{1}, \ldots, q_{r}\right)$, where $\chi\left(x_{1}, \ldots, x_{r}\right)$ is a formula of $\bar{L}$ with parameters in $k$. It follows from 8.1 that (30), (31) and (32) hold in $\tilde{k}$ for some $\left\langle\bar{p}_{1}, \ldots, \bar{p}_{r}\right\rangle \in V \cap k^{r}$ in place of $\left\langle q_{1}, \ldots, q_{r}\right\rangle$. However, we may also apply $(29)$ in $\tilde{k}$ with $p_{i}=a_{i}$ and $t_{i}=\bar{p}_{i}^{\prime}-a_{i}$. (Note that $\left\langle t_{1}, \ldots, t_{m}\right\rangle \in B_{\varepsilon}(0)$ by the new (31) and $(32)$, and $\left\langle a_{1}+t_{1}, \ldots, a_{m}+t_{m}\right\rangle \in B_{\varepsilon}\left(\left\langle a_{1}, \ldots, a_{m}\right\rangle\right) \cap[0,1]^{m}$ since $\left\langle\bar{p}_{1}, \ldots, \bar{p}_{r}\right\rangle \in V$, 
where all these notions are being interpreted in $\tilde{k}$.) Combining this with the new (30) (and using the new (32)) gives:

$$
\begin{aligned}
\left|F\left(\bar{p}_{1}^{\prime}, \ldots, \bar{p}_{m}^{\prime}\right)-p_{e}\right| & <2 C_{\lambda} \cdot \bar{p}_{1}^{-\theta\left(\lambda_{0}+1\right)} \\
& <2 C_{\lambda} \cdot \bar{p}_{1}^{-\mu-1} \quad\left(\text { by choice of } \lambda_{0}\right) \\
& <\bar{p}_{1}^{-\mu} \quad(\text { by the new }(31)),
\end{aligned}
$$

i.e. $\left|g_{r}\left(\bar{p}_{1}, \ldots, \bar{p}_{r}\right)\right|<\bar{p}_{1}^{-\mu}$ is true in $\tilde{k}$. Since $\bar{p}_{1}>B^{\prime}$ (by the new (31)), this contradicts 8.5 and establishes 2.9 .

The proof of the first main theorem is now complete.

\section{TOWARdS THE PROOF OF THE SECOND MAIN THEOREM}

Recall that this states that the theory of the structure $\langle\overline{\mathbb{R}} ; \exp \rangle$ is model complete. Here, exp denotes the exponential function $x \mapsto e^{x}$ defined for all $x \in \mathbb{R}$ and, as before, $\overline{\mathbb{R}}$ denotes the ordered field of real numbers (in the language of ordered rings). Let us denote the theory and language of $\langle\overline{\mathbb{R}} ; \exp \rangle$ by $T_{\exp }$ and $L_{\exp }$ respectively. Then, by the brief discussion of model completeness in section 1 , we must show that if $k, K \models T_{\exp }$ and $k$ is a substructure of $K$, then any existential sentence with parameters in $k$ which is true in $K$ is also true in $k$.

Let us fix $k, K \models T_{\exp }$ with $k$ a substructure of $K$ for the rest of this section. Henceforth, I shall not distinguish notationally between structures and their domains, nor between terms of a language and their interpretations in given structures.

Now consider Theorem 7.2 in the case $m=l=1, C=\emptyset, H_{1}=\exp , \widetilde{K}^{\prime}=K$ and $\tilde{k}^{\prime}=k$. This result tells us that it is sufficient to show that whenever $n \in \mathbb{N}$ and $f_{1}, \ldots, f_{n} \in k\left[x_{1}, \ldots, x_{n}, \exp \left(x_{1}\right), \ldots, \exp \left(x_{n}\right)\right]$ then there exists $b \in k$ such that if $\vec{\alpha}=\left\langle\alpha_{1}, \ldots, \alpha_{n}\right\rangle \in K^{n}$ satisfies $f_{1}(\vec{\alpha})=\cdots=f_{n}(\vec{\alpha})=0$ and $J\left(f_{1}, \ldots, f_{n}\right)(\vec{\alpha}) \neq 0$ (where, as before, $J\left(f_{1}, \ldots, f_{n}\right)$ denotes the determinant of the Jacobian matrix $\left.\left(\frac{\partial f_{i}}{\partial x_{j}}\right)_{1 \leq i, j \leq n}\right)$, then $\left|\alpha_{i}\right|<b$ for $i=1, \ldots, n$.

(This reduction of the problem of proving the model completeness of $\langle\overline{\mathbb{R}} ; \exp \rangle$ was already established in [16] (Theorem 2).)

We shall prove this by induction on the number of exponentials actually occurring in $f_{1}, \ldots, f_{n}$. However, in eliminating an exponential we shall introduce new variables and their exponentials but in such a way that only values of the new variables lying between 0 and 1 will be relevant. This will cause no problems at the base step of the induction because of the model completeness of the structure $\langle\overline{\mathbb{R}} ; \exp \lceil[0,1]\rangle$ (which follows from the first main theorem - see section 1, example (A)). Now it turns out to be technically more convenient to avoid the use of truncated functions, so I define the function $e$ (in any model of $\left.T_{\exp }\right)$ by $e(x)=\exp \left(\left(1+x^{2}\right)^{-1}\right)$ (see section 1, example (A) again). We are thus led to the following

9.1. Definition. Let $n \in \mathbb{N}, s \subseteq\{1, \ldots, n\}$. Then $M_{n}^{s}$ denotes the ring of functions from $K^{n}$ to $K$ generated (as a ring) over $k$ (considered as a field of constant functions) by $x_{i},\left(1+x_{i}^{2}\right)^{-1}, e\left(x_{i}\right)$ (for $\left.i=1, \ldots, n\right)$ and $\exp \left(x_{i}\right)$ (for $i \in s$ ).

Notice that, for any $n \in \mathbb{N}$ and $s \subseteq\{1, \ldots, n\}, M_{n}^{s}$ is a Noetherian ring of $K$-definable, $C^{\infty}$ (in the sense of $K$ ) functions from $K^{n}$ to $K$. Further, $M_{n}^{s}$ is closed under differentiation and so, in particular, for any $f_{1}, \ldots, f_{n} \in M_{n}^{s}$ we have 
$J\left(f_{1}, \ldots, f_{n}\right) \in M_{n}^{s}$. The results of sections 4 and 5 are therefore applicable and we summarize them now in a form suitable for application here.

9.2. Proposition. Let $n \in \mathbb{N}, s \subseteq\{1, \ldots, n\}$.

(i) Suppose $f \in M_{n}^{s}, \vec{\alpha} \in K^{n}$ and $f(\vec{\alpha})=0$. Then there exist $f_{1}, \ldots, f_{n} \in M_{n}^{s}$ and $\vec{\beta} \in K^{n}$ such that $f(\vec{\beta})=f_{1}(\vec{\beta})=\cdots=f_{n}(\vec{\beta})=0$ and $J\left(f_{1}, \ldots, f_{n}\right)(\vec{\beta}) \neq$ 0 .

(ii) If, in (i), $\vec{\alpha}$ is an isolated zero of $f$, then we may take $\vec{\beta}=\vec{\alpha}$.

(iii) Let $f_{1}, \ldots, f_{n} \in M_{n}^{s}$. Then there are only finitely many $\vec{\gamma} \in K^{n}$ such that $f_{1}(\vec{\gamma})=\cdots=f_{n}(\vec{\gamma})=0$ and $J\left(f_{1}, \ldots, f_{n}\right)(\vec{\gamma}) \neq 0$.

Proof. For (i) apply Theorem 5.1 with $\overline{\bar{T}}=T_{\exp }, \overline{\bar{K}}=K, M=M_{n}^{s}, U=K^{n}$ and $S=\left\{\vec{\gamma} \in K^{n}: f(\vec{\gamma})=0\right\}(=V(f))$.

For (ii) apply Theorem 4.9 (with $\overline{\bar{T}}=T_{\exp }, \overline{\bar{K}}=K, P_{0}=\vec{\alpha}$ and $M=\{[g\lceil U, U]$ : $U$ an open neighbourhood of $\vec{\alpha}$ in $K^{n}$ and $\left.g \in M_{n}^{s}\right\}$ ) repeatedly for $m=0, \ldots, n-1$. We must eventually find $f_{1}, \ldots, f_{n} \in M_{n}^{s}$ such that $\vec{\alpha} \in V^{n s}\left(f_{1}, \ldots, f_{n}\right)$ because otherwise we would have (ii) of Theorem 4.9 holding for some $f_{1}, \ldots, f_{m}$ with $m<n$ and, in particular, for $[h, W]=\left[f, K^{n}\right]$. But this contradicts the implicit function theorem applied in $K$ (see the beginning of section 4 ) and the fact that $\vec{\alpha}$ is an isolated zero of $f$.

Finally, note that the sequence $\left(1+x_{1}^{2}\right)^{-1}, \ldots,\left(1+x_{n}^{2}\right)^{-1}, e\left(x_{1}\right), \ldots, e\left(x_{n}\right)$, $\exp \left(x_{i_{1}}\right), \ldots, \exp \left(x_{i_{m}}\right)$ (where $\left.s=\left\{i_{1}, \ldots, i_{m}\right\}\right)$ is a Pfaffian chain on $\mathbb{R}^{n}$. Statement (iii) follows upon transferring Theorem 3.1 to $K$.

Let us now assume that the second main theorem is false. By the discussion above it follows that there exists $m \in \mathbb{N}$ such that:

for some $n \in \mathbb{N}, n \geq m$, there exist $\vec{\alpha}=\left\langle\alpha_{1}, \ldots, \alpha_{n}\right\rangle \in K^{n}$,

$l \in\{1, \ldots, n\}$ and $s \subseteq\{1, \ldots, n\}$ with $\operatorname{card}(s)=m$ such that for some

$f_{1}, \ldots, f_{n} \in M_{n}^{s}, f_{1}(\vec{\alpha})=\cdots=f_{n}(\vec{\alpha})=0 \neq J\left(f_{1}, \ldots, f_{n}\right)(\vec{\alpha})$. Further,

$\left|\alpha_{l}\right|>b$ for all $b \in k$, and if $m>0$, then $l \in s$.

(Of course, the comments above imply that we could take $n=m$ and $s=$ $\{1, \ldots, n\}$ here, but, as already mentioned, the point is that we shall be reducing $m$ at the expense of extra variables and $e$-terms.)

Choose $m$ minimal such that $(*)_{m}$ holds. I first claim that $m>0$. For consider the structure with the same domain and ordered ring structure as $K$, but with exp replaced by $e$. Call the resulting structure $K^{\prime}$ and proceed similarly to obtain $k^{\prime}$ from $k$. Clearly $k^{\prime}$ is a substructure of $K^{\prime}$ and they are both models of the complete theory of the structure $\left\langle\overline{\mathbb{R}} ; x \mapsto \exp \left(\left(1+x^{2}\right)^{-1}\right)\right\rangle$. But by example (A) of section 1 , this theory is model complete. This contradicts $(*)_{0}$ and 9.2 (iii).

Now for our minimal (non-zero) $m$, choose $n, \vec{\alpha}, l, s$ and $f_{1}, \ldots, f_{n}$ witnessing $(*)_{m}$. In the final section of this paper I shall establish (independently of all assumptions being made here) a property of elements of models of $T_{\exp }$ and their exponentials which implies the following:

9.3. There exist integers $n_{i}$ (for $i \in s$ ), not all zero, and $c \in k$ such that $0<$ $c+\sum_{i \in s} n_{i} \alpha_{i}<1$.

Assuming 9.3, note that since $\left|\alpha_{l}\right|>b$ for all $b \in k$, we cannot have $n_{i}=0$ for all $i \in s \backslash\{l\}$. Suppose, for convenience, that $1 \in s, n_{1} \neq 0$, and $1 \neq l$. We may 
assume that $n_{1}>0$ for if $n_{1}<0$ simply replace $n_{i}$ by $-n_{i}$ (for $i \in s$ ) and $c$ by $1-c$ in 9.3. Now set $\alpha_{n+1}=\exp \left(\alpha_{1}\right)$ and choose $\alpha_{n+2} \in K$ so that $\alpha_{n+2}>0$ and $\left(1+\alpha_{n+2}^{2}\right)^{-1}=c+\sum_{i \in s} n_{i} \alpha_{i}$. This is possible since $K$, as a field, is real closed.

Now let $g_{i}\left(x_{1}, \ldots, x_{n+1}\right)$ be the result of replacing $\exp \left(x_{1}\right)$ by $x_{n+1}$ in $f_{i}\left(x_{1}, \ldots, x_{n}\right)$. Then $g_{i} \in M_{n+1}^{s \backslash\{1\}}$ and clearly $\left\langle\alpha_{1}, \ldots, \alpha_{n+2}\right\rangle$ is a solution of the following system of equations:

$$
\Lambda\left(x_{1}, \ldots, x_{n+2}\right):\left\{\begin{array}{l}
g_{1}\left(x_{1}, \ldots, x_{n+1}\right)=0, \\
\vdots \\
g_{n}\left(x_{1}, \ldots, x_{n+1}\right)=0, \\
\left(1+x_{n+2}^{2}\right)^{-1}-c-\sum_{i \in s} n_{i} x_{i}=0, \\
x_{n+1}^{n_{1}} \cdot \exp (c) \cdot \prod_{j \in s^{+}} \exp \left(x_{j}\right)^{n_{j}} \\
\quad-e\left(x_{n+2}\right) \cdot \prod_{j \in s^{-}} \exp \left(x_{j}\right)^{-n_{j}}=0,
\end{array}\right.
$$

where $s^{ \pm}=\left\{j \in s: j>1\right.$ and $\left.\pm n_{j}>0\right\}$ (respectively). (The last equation is obtained by exponentiating the previous one, substituting $x_{n+1}$ for $\exp \left(x_{1}\right)$ and rearranging. An empty product is interpreted as 1.)

Now by 9.2 (iii) there exists a $K$-definable open neighbourhood, $U$ say, of $\left\langle\alpha_{1}, \ldots, \alpha_{n}\right\rangle$ (in $K^{n}$ ) such that $\left\langle\alpha_{1}, \ldots, \alpha_{n}\right\rangle$ is the only solution of $f_{1}\left(x_{1}, \ldots, x_{n}\right)=$ $\cdots=f_{n}\left(x_{1}, \ldots, x_{n}\right)=0 \neq J\left(f_{1}, \ldots, f_{n}\right)\left(x_{1}, \ldots, x_{n}\right)$ in $U$. Since

$$
J\left(f_{1}, \ldots, f_{n}\right)\left(\alpha_{1}, \ldots, \alpha_{n}\right) \neq 0
$$

we may actually suppose that $\left\langle\alpha_{1}, \ldots, \alpha_{n}\right\rangle$ is the only solution of $f_{1}\left(x_{1}, \ldots, x_{n}\right)=$ $\cdots=f_{n}\left(x_{1}, \ldots, x_{n}\right)=0$ in $U$. I now claim that $\left\langle\alpha_{1}, \ldots, \alpha_{n+2}\right\rangle$ is the only solution of the system $\Lambda\left(x_{1}, \ldots, x_{n+2}\right)$ lying in the open subset $U \times K^{+} \times K^{+}$of $K^{n+2}$ (where $K^{+}=\{a \in K: a>0\}$ ). For suppose that $\left\langle\beta_{1}, \ldots, \beta_{n+2}\right\rangle$ is such a solution. Since $\beta_{n+1}>0$ and $n_{1} \neq 0$ the last two equations force $\beta_{n+1}=\exp \left(\beta_{1}\right)$. The first $n$ equations now force $f_{i}\left(\beta_{1}, \ldots, \beta_{n}\right)=0$ for $i=1, \ldots, n$ and hence, since $\left\langle\beta_{1}, \ldots, \beta_{n}\right\rangle \in U, \beta_{i}=\alpha_{i}$ for $i=1, \ldots, n$. Further, $\beta_{n+1}=\exp \left(\beta_{1}\right)=$ $\exp \left(\alpha_{1}\right)=\alpha_{n+1}$. Finally, the penultimate equation and the condition $\beta_{n+2}>0$ force $\beta_{n+2}=\alpha_{n+2}$.

Now let $f$ be the sum of the squares of the $n+2$ functions appearing in $\Lambda\left(x_{1}, \ldots, x_{n+2}\right)$. Then $f \in M_{n+2}^{s \backslash\{1\}}$ (note that $c, \exp (c) \in k$ ) and we have shown that $\left\langle\alpha_{1}, \ldots, \alpha_{n+2}\right\rangle$ is an isolated zero of $f$. By 9.2 (ii) it follows that there exist $h_{1}, \ldots, h_{n+2} \in M_{n+2}^{s \backslash\{1\}}$ such that $h_{1}\left(\alpha_{1}, \ldots, \alpha_{n+2}\right)=\cdots=h_{n+2}\left(\alpha_{1}, \ldots, \alpha_{n+2}\right)=$ $0 \neq J\left(h_{1}, \ldots, h_{n+2}\right)\left(\alpha_{1}, \ldots, \alpha_{n+2}\right)$. Since $l \in s \backslash\{1\}$, this implies that $(*)_{m-1}$ holds which contradicts the minimality of $m$ and establishes the second main theorem modulo 9.3 .

\section{Smooth 0-Minimal theories}

We touched on the notion of 0-minimality in section 2 where it was needed to establish asymptotic formulas for definable functions in structures covered by the first main theorem. We now require a deeper asymptotic analysis and I must assume that the reader is familiar with the basic general properties of 0 -minimal structures. These can be found in the foundational papers [10] and [7]. (See also [15] for more recent developments.)

For this section let $\widetilde{\mathbb{R}}$ be any 0-minimal expansion of the real ordered field $\overline{\mathbb{R}}$ and let $\widetilde{T}$ denote the complete theory of $\widetilde{\mathbb{R}}$. Then $\widetilde{T}$ admits definable Skolem 
functions and the closure of $\{0\}$ under these functions in any model $K$ of $\widetilde{T}$ is an Archimedean-ordered elementary substructure of $K$ (because $\widetilde{T}$ is complete and has an Archimedean ordered model).

Suppose that $K \models \widetilde{T}, k \preceq K$ (i.e. $k$ is an elementary substructure of $K$ ) and $n \in \mathbb{N}$.

I shall say that a function from $K^{n}$ to $K$ or a subset of $K^{n}$ is $k$-definable if it is definable by a formula of the language of $\widetilde{T}$ possibly involving parameters from $k$.

Consider now the following condition on $\widetilde{T}$ :

(S1) For any $K \models \widetilde{T}$ and any $K$-definable function $f: K \rightarrow K$, there exists $N \in \mathbb{N}$ such that $|f(x)| \leq x^{N}$ for all sufficiently large $x \in K$.

10.1. Theorem. Suppose $\widetilde{T}$ satisfies (S1). Let $K \models \widetilde{T}$ and suppose that $R$ is a convex subring of $K$. Let I be the (convex) ideal of $R$ consisting of those elements of $R$ which are not invertible in $R$ (i.e. $I$ is the unique maximal ideal of $R$ ). Then there exists $k_{0} \preceq K$ such that $k_{0} \subseteq R$ and such that for each $a \in R, k_{0} \cap(a+I)$ contains exactly one element, i.e. $k_{0}$ splits $R$.

Proof. Clearly the set $\mathcal{S}=\{k: k \preceq K$ and $k \subseteq R\}$ satisfies the hypotheses of Zorn's lemma ( $\mathcal{S}$ is nonempty since it contains the Skolem closure of $\{0\}$ in $K$ ). Let $k_{0}$ be a maximal element of $\mathcal{S}$. Then $k_{0} \preceq K$ (so $\left.k_{0}=\widetilde{T}\right), k_{0} \subseteq R$ and clearly, since $k_{0}$ is a field, $k_{0} \cap(a+I)$ contains at most one element for each $a \in R$.

I claim that for all $a \in R$ there exists $\alpha \in k_{0}$ such that $\alpha>a$. For suppose $a$ is a counterexample. Since $\widetilde{T}$ has definable Skolem functions the set $\{f(a): f:$ $K \rightarrow K, f$ a $k_{0}$-definable function $\}$ is the domain of an elementary substructure of $K$ (containing $k_{0}$ ) which, by the maximality of $k_{0}$, contains an element greater than every element of $R$. Suppose $f(a)$ is such an element (where $f: K \rightarrow K$ is $k_{0}$-definable). By (S1) there is an element $b \in k_{0}$ and $N \in \mathbb{N}$ such that $k_{0}=$ $\forall x>b\left(|f(x)| \leq x^{N}\right.$ ). Since $k_{0} \preceq K$ and $a>b$ we have $|f(a)| \leq a^{N}$ (in $K$ ), contradicting the fact that $R$ is a subring of $K$.

Now suppose that $a \in R$ and that $k_{0} \cap(a+I)=\emptyset$. It is again sufficient (for a contradiction) to show that $f(a) \in R$ for any $k_{0}$-definable function $f: K \rightarrow K$. So let $f$ be such a function. By a result of [10] there are elements $a_{1}<a_{2}<\cdots<a_{n}$ of $k_{0}$ such that (setting $\left.a_{0}=-\infty, a_{n+1}=+\infty\right) f$ is (weakly) monotonic (in $k_{0}$, and hence in $K$ ) on each open interval $\left(a_{i}, a_{i+1}\right)$ for $i=0, \ldots, n$. Thus, by the claim above, there exist $b, c \in k_{0}$ with $b<a<c$ and $f$ (weakly) monotonic on $(b, c)$. Since $k_{0} \cap(a+I)=\emptyset$ we have $c-a, a-b>\beta$ for all $\beta \in I$, and hence $(c-a)^{-1}$, $(a-b)^{-1} \in R$. By the claim there exists $d \in k_{0}$ such that $d>(c-a)^{-1},(a-b)^{-1}$. But then $d^{-1} \in k_{0}$ and $b<b+d^{-1}<a<c-d^{-1}<c$. It follows that $f(a)$ lies between the elements $f\left(b+d^{-1}\right), f\left(c-d^{-1}\right)$ of $k_{0}$ and hence $f(a) \in R$, as required.

Let $K \models \widetilde{T}$. For any subset $A$ of $K$ denote by $\mathcal{C} \ell(A)$ the closure of $A$ (in $K$ ) under the Skolem functions of $\widetilde{T}$. The set $A$ is said to generate $K$ if $\mathcal{C} \ell(A)=K$, and is called independent if $a \notin \mathcal{C} \ell(A \backslash\{a\})$ for each $a \in A$. An independent set that generates $K$ is called a basis for $K$. It was shown in [10] that this notion of independence has the exchange property, and hence any independent subset of $K$ can be extended to a basis for $K$ and all bases for $K$ have the same cardinality. The cardinality of any basis for $K$ is denoted $\operatorname{dim}(K)$. 
If $k \preceq K$, then all the above remarks apply "over $k$ ", i.e. to the notion of closure under $k$-definable functions. I denote by $\operatorname{dim}_{k}(K)$ the cardinality of any basis for $K$ over $k$. If $k_{0} \preceq k_{1} \preceq K$ it is easy to verify that $\operatorname{dim}_{k_{0}}(K)=\operatorname{dim}_{k_{0}}\left(k_{1}\right)+\operatorname{dim}_{k_{1}}(K)$ provided that $\operatorname{dim}_{k_{0}}(K)$ is finite.

I now introduce another notion of dimension for models of $\widetilde{T}$. Indeed, let $K$ be any real-closed ordered field. Recall that an element $a$ of $K$ is called finite if $|a|<n$ for some $n \in \mathbb{N}$, and infinitesimal if $|a|<\frac{1}{n}$ for all $n \in \mathbb{N} \backslash\{0\}$. The set $\operatorname{Fin}(K)$ of finite elements of $K$ forms a convex subring of $K$ with unique maximal ideal $\mu(K)$, the set of infinitesimals. Further, the set Fin $(K) \backslash \mu(K)$ is a multiplicative subgroup of $K \backslash\{0\}$, and I call the quotient of the latter by the former the value group of $K$ and denote it by $V(K)$. It is usual to write $V(K)$ as an additive group and as such it can be ordered by setting $a /(\operatorname{Fin}(K) \backslash \mu(K))>0$ if and only if $a \in \mu(K)$. Further, $V(K)$ is a divisible group (since $m$ th roots of positive elements exist in $K$ for all $m \in \mathbb{N} \backslash\{0\})$ and is therefore an ordered $\mathbb{Q}$-vector space. I denote its dimension over $\mathbb{Q}$ by valdim $(K)$.

The map $\nu_{K}: K \rightarrow V(K) \cup\{\infty\}$, extending the natural map $K \backslash\{0\} \rightarrow V(K)$ by setting $\nu_{K}(0)=\infty$, is called the valuation map of $K$. It is easy to verify the following (where we set $\infty>\alpha$ for all $\alpha \in V(K)$ and $\infty+\alpha=\alpha+\infty=\infty$ for all $\alpha \in V(K) \cup\{\infty\})$ :

(v1) $\nu_{K}(x \cdot y)=\nu_{K}(x)+\nu_{K}(y)$ for all $x, y \in K$;

(v2) $\nu_{K}(x+y) \geq \min \left(\nu_{K}(x), \nu_{K}(y)\right)$ for all $x, y \in K$, with equality if $\nu_{K}(x) \neq$ $\nu_{K}(y)$

(v3) for all $x \in K, \nu_{K}(x) \geq 0$ if and only if $x \in \operatorname{Fin}(K)$, and $\nu_{K}(x)>0$ if and only if $x \in \mu(K)$.

My present aim is to formulate a condition on $\widetilde{T}$ that guarantees (if $\widetilde{T}$ also satisfies (S1)) that valdim $(K) \leq \operatorname{dim}(K)$ for all models $K$ of $\widetilde{T}$ for which $\operatorname{dim}(K)$ is finite. Notice that this inequality is satisfied if $\widetilde{T}$ is just the theory of real-closed ordered fields (i.e. $\widetilde{\mathbb{R}}=\overline{\mathbb{R}}$ ). For in this case $\operatorname{dim}(K)$ is the transcendence degree (over $\mathbb{Q})$ of $K$ and it is easy to check that if $\alpha_{1}, \ldots, \alpha_{n} \in K$ and $p\left(\alpha_{1}, \ldots, \alpha_{n}\right)=0$ for some non-trivial polynomial $p$ with rational coefficients, then $\nu_{K}\left(\alpha_{1}\right), \ldots, \nu_{K}\left(\alpha_{n}\right)$ are linearly dependent over $\mathbb{Q}$.

Consider the following condition on $\widetilde{T}$ :

(S2) For any formula $\phi\left(x_{1}, \ldots, x_{n}\right)$ of the language of $\widetilde{T}$ there are $m, p \in \mathbb{N}$ and $C^{\infty}$ functions $F_{i}: \mathbb{R}^{n+m} \rightarrow \mathbb{R}$ (for $i=1, \ldots, p$ ), which are definable without parameters in $\widetilde{\mathbb{R}}$, and are such that

$$
\widetilde{\mathbb{R}}=\forall \vec{x}\left(\phi(\vec{x}) \leftrightarrow \exists \vec{y}\left(\|\vec{y}\| \leq 1 \wedge \bigvee_{i=1}^{p}\left(N_{i}(\vec{y}) \wedge F_{i}(\vec{x}, \vec{y})=0\right)\right),\right.
$$

where, if $\vec{y}=y_{1}, \ldots, y_{m},\|\vec{y}\|=\max \left\{\left|y_{i}\right|: i=1, \ldots, m\right\}$ and $N_{i}(\vec{y})$ is a formula of the form $\bigwedge_{j \in s_{i}} y_{j} \neq 0$ for some $s_{i} \subseteq\{1, \ldots, m\}$.

10.2. Definition. If $\widetilde{T}$ satisfies (S1) and (S2) (and $\widetilde{T}$ is the complete theory of a 0 -minimal expansion $\widetilde{\mathbb{R}}$ of $\mathbb{R}$ ), then $\widetilde{T}$ is called smooth.

10.3. Theorem. Suppose $\widetilde{T}$ is smooth and $K \models \widetilde{T}$. If $\operatorname{dim}(K)$ is finite, then $\operatorname{valdim}(K) \leq \operatorname{dim}(K)$. 
Proof. The proof is by induction on $\operatorname{dim}(K)$.

If $K$ is Archimedean (i.e. $\mu(K)=\{0\}$ ), then valdim $(K)=0$ so the result is clear. Notice that this covers the case $\operatorname{dim}(K)=0$ (by the remarks at the beginning of this section), so suppose that $\operatorname{dim}(K)=n>0$ and $\mu(K) \neq\{0\}$.

By an argument similar to the one used in the claim in the proof of 10.1, there is some $a_{0} \in \mu(K)$ with $a_{0}>0$ such that for all $b \in K$ with $b>0$ we have $a_{0}^{m}<b$ for some $m \in \mathbb{N}$. Let $R=\left\{b \in K:|b|<a_{0}^{-1 / m}\right.$ for all $\left.m \in \mathbb{N} \backslash\{0\}\right\}$. Then $R$ is a convex subring of $K$ and its maximal ideal, $I$ say, is Archimedean in the sense that for all $a, b \in I \backslash\{0\}$, there is some $m \in \mathbb{N}$ such that $|b|^{m}<|a|$.

By 10.1 we may choose $k \preceq K$ such that $k$ splits $R$. Since $k \neq K$ we have $\operatorname{dim}(k)<n$. Say $\operatorname{dim}(i)=n-r($ where $r \in \mathbb{N} \backslash\{0\})$ and choose $c_{1}, \ldots, c_{r} \in K$ such that $\left\{c_{1}, \ldots, c_{r}\right\}$ is a basis for $K$ over $k$. We may suppose that $c_{1}, \ldots, c_{r} \in I$ since if $c_{i} \notin R$ then we may replace $c_{i}$ by $c_{i}^{-1}$, and if $c_{i} \in R$ we may replace $c_{i}$ by the unique $\eta \in I$ such that $c_{i}+\eta \in k$ (using the splitting property of $k$ ).

Now let $k^{*}$ be the algebraic closure of the field $k\left(c_{1}, \ldots, c_{r}\right)$ in $K$. Then clearly $\nu_{K}\left[k^{*}\right]$ is a subspace of $V(K)$ and by an argument similar to the one discussed before the formulation of $(\mathrm{S} 2)$ we have $\operatorname{dim}_{\mathbb{Q}} \nu_{K}\left[k^{*}\right] \leq \operatorname{dim}_{\mathbb{Q}} \nu_{K}[k]+r$ (where $\operatorname{dim}_{\mathbb{Q}}$ means $\mathbb{Q}$-vector space dimension here). However, clearly $\nu_{K}[k] \cong V(k)$ (as $\mathbb{Q}$-vector spaces) so $\operatorname{dim}_{\mathbb{Q}} \nu_{K}\left[k^{*}\right] \leq \operatorname{valdim}(k)+r$ which, by the inductive hypothesis, implies $\operatorname{dim}_{\mathbb{Q}} \nu_{K}\left[k^{*}\right] \leq \operatorname{dim}(k)+r=n$. Hence it is sufficient to show that $\nu_{K}$ maps $k^{*} \backslash\{0\}$ surjectively onto $V(K)$.

Let $d \in K \backslash\{0\}$. We must show that there is some $\alpha \in k^{*}$ such that $\nu_{K}(\alpha)=$ $\nu_{K}(d)$. Since $\nu_{K}(-\beta)=\nu_{K}(\beta)$ and $\nu_{K}\left(\beta^{-1}\right)=-\nu_{K}(\beta)$ for any $\beta \in K \backslash\{0\}$, and $\nu_{K}(\beta) \in \nu_{K}[k]$ for any $\beta \in R \backslash I$ (as $k$ splits $R$ ), we may suppose that $d>0$ and $d \in I$. Let $f: K^{r} \rightarrow K$ be a $k$-definable function such that $f\left(c_{1}, \ldots, c_{r}\right)=d$.

By (S2) there exists a $k$-definable, $C^{\infty}$ (in the sense of $K$ ) function $F: K^{r+1+m} \rightarrow$ $K$ (for some $m \in \mathbb{N}$ ) and $s \subseteq\{1, \ldots, m\}$ such that:

$$
\begin{aligned}
& \text { for all } x \in K, f\left(c_{1}, \ldots, c_{r}\right)=x \text { if and only if there exist } \\
& b_{1}, \ldots, b_{m} \in K \text { with } b_{i} \neq 0 \text { for } i \in s, \text { and }\left|b_{i}\right| \leq 1 \text { for } i=1, \ldots, m \\
& \text { such that } F\left(c_{1}, \ldots, c_{r}, x, b_{1}, \ldots, b_{m}\right)=0 .
\end{aligned}
$$

(In applying (S2) I have replaced the parameters from $k$ occurring in the formula defining he graph of $f$ by variables, to obtain $\phi\left(\vec{z}, x_{1}, \ldots, x_{r}, x\right)$ say, and then taken $F$ to be that $F_{i}$ for which the corresponding disjunct holds in $K$ when these parameters are replaced for $\vec{z}$ in $\phi$, and $x_{i}$ is set to $c_{i}$ for $i=1, \ldots, r$ and $x$ is set to $d$.)

Now fix $\beta_{1}, \ldots, \beta_{m} \in K$ such that $\beta_{i} \neq 0$ for $i \in s,\left|\beta_{i}\right| \leq 1$ for $i=1, \ldots, m$, and $F\left(c_{1}, \ldots, c_{r}, d, \beta_{1}, \ldots, \beta_{m}\right)=0$. Since $\beta_{1}, \ldots, \beta_{m} \in R$ we may choose (uniquely) $\beta_{1}^{0}, \ldots, \beta_{m}^{0} \in k$ such that $\beta_{i}-\beta_{i}^{0} \in I$ for $i=1, \ldots, m$ (using the splitting property of $k$ ). Further, by the Archimedean property of $I$ we may choose $N \in \mathbb{N}$ so large that $\left|\beta_{i}\right|>\left|c_{1}\right|^{N}$ for $i \in s$. (We cannot have $c_{1}=0$ since $c_{1}$ occurs in a basis for $K$ over $k$.)

Let $A=\left\{\left\langle x_{1}, \ldots, x_{m}\right\rangle \in K^{m}:\left|c_{1}\right|^{N} \leq\left|x_{i}\right|\right.$ for $i \in s,\left|x_{i}\right| \leq 1$ for $\left.i=1, \ldots, m\right\}$.

Consider the function

$$
h: K^{1+m} \rightarrow K:\left\langle x, x_{1}, \ldots, x_{m}\right\rangle \mapsto\left|F\left(c_{1}, \ldots, c_{r}, x, x_{1}, \ldots, x_{m}\right)\right| .
$$

Since (in the sense of $K$ ) $h$ is continuous, it must achieve its minimum on any closed, bounded, $K$-definable subset of $K^{1+m}$. Let $\gamma$ be the minimum of $h$ on 
$\left([0,1] \backslash\left(\frac{d}{2}, \frac{3 d}{2}\right)\right) \times A$. By (33) and the preceding remark we have that $\gamma>0$, so we may choose $N^{\prime} \in \mathbb{N}$ so large that $\gamma>\left|c_{1}\right|^{N^{\prime}}$. Then clearly:

$$
\begin{aligned}
& \text { for all } \alpha \in[0,1] \text { and }\left\langle\beta_{1}^{\prime}, \ldots, \beta_{m}^{\prime}\right\rangle \in A \text {, } \\
& \text { if }\left|F\left(c_{1}, \ldots, c_{r}, \alpha, \beta_{1}^{\prime}, \ldots, \beta_{m}^{\prime}\right)\right| \leq\left|c_{1}\right|^{N^{\prime}}, \\
& \text { then } \frac{d}{2}<\alpha<\frac{3 d}{2} \text { and hence } \nu_{K}(\alpha)=\nu_{K}(d) .
\end{aligned}
$$

Now let $\lambda \in \mathbb{N}$ and consider the Taylor expansion to degree $\lambda$ of the function $F: K^{r+1+m} \rightarrow K$, with Lagrange's form of the remainder, about the point

$$
\vec{\omega}=\langle\underbrace{0, \ldots, 0}_{r+1}, \beta_{1}^{0}, \ldots, \beta_{m}^{0}\rangle\left(\in k^{r+1+m}\right) .
$$

This clearly provides us with (by either transferring the classical result from $\widetilde{\mathbb{R}}$ to $K$, or else by just proving Taylor's theorem in $K$ ) a polynomial

$$
\rho_{\lambda}\left(y_{1}, \ldots, y_{r}, x, x_{1}, \ldots, x_{m}\right)
$$

with coefficients in $k$ and an element $B_{\lambda} \in k\left(B_{\lambda}>0\right)$ such that:

$$
\begin{aligned}
& \text { for all } t \in K \text { with } 1>t>0, \text { and all } \\
& \vec{z} \in K^{r+1+m} \text { with }\|\vec{z}-\vec{\omega}\|<t,\left|F(\vec{z})-\rho_{\lambda}(\vec{z})\right|<B_{\lambda} \cdot t^{\lambda+1} .
\end{aligned}
$$

Let $t_{0}=2 \cdot \max \left(\left|c_{1}\right|, \ldots,\left|c_{r}\right|, d,\left|\beta_{1}-\beta_{1}^{0}\right|, \ldots,\left|\beta_{m}-\beta_{m}^{0}\right|\right)$. Then $t_{0} \in I$ and $t_{0}>0$, so we may choose $\lambda_{0} \in \mathbb{N}$ so large that:

$$
t_{0}^{\lambda_{0}+1}<\left(2 B_{\lambda_{0}}\right)^{-1} \cdot\left|c_{1}\right|^{N^{\prime}} .
$$

Now setting $\lambda=\lambda_{0}, t=t_{0}$ and $\vec{z}=\left\langle c_{1}, \ldots, c_{r}, d, \beta_{1}, \ldots, \beta_{m}\right\rangle$ in (35), and then using (36), gives:

$$
\left|\rho\left(c_{1}, \ldots, c_{r}, d, \beta_{1}, \ldots, \beta_{m}\right)\right|<\frac{1}{2} \cdot\left|c_{1}\right|^{N^{\prime}}, \quad \text { where } \rho=\rho_{\lambda_{0}} .
$$

We also clearly have:

$$
\left\langle d, \beta_{1}, \ldots, \beta_{m}\right\rangle \in[0,1] \times A
$$

and

$$
\left\|\left\langle c_{1}, \ldots, c_{r}, d, \beta_{1}, \ldots, \beta_{m}\right\rangle-\vec{\omega}\right\|<\left(\left(2 B_{\lambda_{0}}\right)^{-1} \cdot\left|c_{1}\right|^{N^{\prime}}\right)^{\left(\lambda_{0}+1\right)^{-1}} .
$$

Now (37), (38) and (39) can be expressed in the language of ordered rings and can be viewed as conditions on the point $\left\langle d, \beta_{1}, \ldots, \beta_{m}\right\rangle$ with parameters in $k^{*}$ (= the algebraic closure of the field $k\left(c_{1}, \ldots, c_{r}\right)$ in $\left.K\right)$. Since $k^{*}$ is an elementary substructure of $K$ for the language of ordered rings (both being real-closed ordered fields) it follows that there are $\alpha, \beta_{1}^{\prime}, \ldots, \beta_{m}^{\prime} \in k^{*}$ such that:

$$
\begin{gathered}
\left|\rho\left(c_{1}, \ldots, c_{r}, \alpha, \beta_{1}^{\prime}, \ldots, \beta_{m}^{\prime}\right)\right|<\frac{1}{2} \cdot\left|c_{1}\right|^{N^{\prime}}, \\
\left\langle\alpha, \beta_{1}^{\prime}, \ldots, \beta_{m}^{\prime}\right\rangle \in[0,1] \times A,
\end{gathered}
$$

and

$$
\left\|\left\langle c_{1}, \ldots, c_{r}, \alpha, \beta_{1}^{\prime}, \ldots, \beta_{m}^{\prime}\right\rangle-\vec{\omega}\right\|<\left(\left(2 B_{\lambda_{0}}\right)^{-1} \cdot\left|c_{1}\right|^{N^{\prime}}\right)^{\left(\lambda_{0}+1\right)^{-1}} .
$$


Now by (42), we may apply (35) with $\lambda=\lambda_{0}, t=\left(\left(2 B_{\lambda_{0}}\right)^{-1} \cdot\left|c_{1}\right|^{N^{\prime}}\right)^{\left(\lambda_{0}+1\right)^{-1}}$ and $\vec{z}=\left\langle c_{1}, \ldots, c_{r}, \alpha, \beta_{1}^{\prime}, \ldots, \beta_{m}^{\prime}\right\rangle$ which, together with (40) gives:

$$
\left|F\left(c_{1}, \ldots, c_{r}, \alpha, \beta_{1}^{\prime}, \ldots, \beta_{m}^{\prime}\right)\right|<\left|c_{1}\right|^{N^{\prime}} .
$$

The required conclusion now follows from (43), (41) and (34).

Suppose $K \models \widetilde{T}$ and $k \preceq K$. Then $\nu_{K}[k \backslash\{0\}]$ is a $\mathbb{Q}$-vector subspace of $V(K)$ (because $k$ is certainly a real-closed ordered subfield of $K$ ) and I denote the dimension of $V(K)$ over $\nu_{K}[k \backslash\{0\}]$ by $\operatorname{valdim}_{k}(K)$.

I require the following generalization of 3.4 .

10.4. Theorem. Suppose $\widetilde{T}$ is smooth, $K \models \widetilde{T}, k \preceq K$ and that $\operatorname{dim}_{k}(K)$ is finite. Then $\operatorname{valdim}_{k}(K) \leq \operatorname{dim}_{k}(K)$.

Proof. It is clearly sufficient to consider the case $\operatorname{dim}_{k}(K)=1$, so let $a$ be a generator for $K$ over $k$. Suppose, for a contradiction, that valdim $\lim _{k}(K) \geq 2$. Then there exist $k$-definable functions $f, g: K \rightarrow K$ such that $\nu_{K}(f(a)), \nu_{K}(g(a))$ are $\mathbb{Q}$-linearly independent over $\nu_{K}[k \backslash\{0\}]$.

Now consider the structure $\langle K, P\rangle$, where $P$ is the unary relation on $K$ interpreted as (the domain of) $k$. Let $\left\langle{ }^{*} K,{ }^{*} P\right\rangle$ be an $\aleph_{0}$-saturated elementary extension of $\langle K, P\rangle$. Let ${ }^{*} k$ be the elementary substructure of ${ }^{*} K$ with domain ${ }^{*} P$. Then ${ }^{*} k$ is certainly $\aleph_{0}$-saturated. I claim that $\nu_{*}(f(a)), \nu_{*}(g(a))$ are $\mathbb{Q}$-linearly independent over $\left.\nu_{*}{ }^{[*} k \backslash\{0\}\right]$. For suppose not. Then for some $p, q \in \mathbb{Q}$ not both zero, and some $b \in{ }^{*} k \backslash\{0\}$ we have $p \nu_{*}(f(a))+q \nu_{*}(g(a))+\nu_{* k}(b)=0$. This implies that $i^{-1}<|f(a)|^{p} \cdot|g(a)|^{q} \cdot|b|<i$ for some $i \in \mathbb{N} \backslash\{0\}$. Since $a \in K$ and $\langle K, P\rangle \preceq\left\langle{ }^{*} K,{ }^{*} P\right\rangle$ it follows that there is some $b^{\prime} \in k \backslash\{0\}$ such that

$$
i^{-1}<|f(a)|^{p} \cdot|g(a)|^{q} \cdot\left|b^{\prime}\right|<i
$$

which contradicts the fact that $\nu_{K}(f(a)), \nu_{K}(g(a))$ are $\mathbb{Q}$-linearly independent over $\nu_{K}[k \backslash\{0\}]$.

This shows that we may suppose that $k$ is $\aleph_{0}$-saturated (by taking $k={ }^{*} k$ and $K$ to be the elementary substructure of ${ }^{*} K$ generated over ${ }^{*} k$ by $a$ ).

Now let $k_{0}$ be an elementary substructure of $k$ such that $\operatorname{dim}\left(k_{0}\right)$ is finite and such that $f, g$ are both $k_{0}$-definable. Consider the following set of formulas over $k$ :

$$
\begin{aligned}
\Theta(x): \quad\left\{|f(x)|^{p} \cdot|g(x)|^{q} \cdot|b|\right. & \leq i^{-1} \vee|f(x)|^{p} \cdot|g(x)|^{q} \cdot|b| \geq i: \\
i & \left.\in \mathbb{N} \backslash\{0\}, p, q \in \mathbb{Q}, \text { not both zero, } b \in k_{0} \backslash\{0\}\right\} .
\end{aligned}
$$

Clearly $\Theta(x)$ is realised in $K$ by $a$ and hence $\Theta(x)$ is finitely satisfiable in $k$. Further, since $\operatorname{dim}\left(k_{0}\right)$ is finite, $\Theta(x)$ can be rewritten so that it contains only finitely many parameters from $k_{0}$ (namely, the elements of a basis) and hence from $k$. Thus $\Theta(x)$ is realized in $k$, by $a_{1}$ say. Let $k_{1}$ be the elementary substructure of $k$ generated over $k_{0}$ by $a_{1}$. Then clearly $\operatorname{dim}\left(k_{1}\right)=\operatorname{dim}\left(k_{0}\right)+1$ and $\operatorname{valdim}\left(k_{1}\right) \geq \operatorname{valdim}\left(k_{0}\right)+2$ (since $\nu_{k_{1}}\left(f\left(a_{1}\right)\right), \nu_{k_{1}}\left(g\left(a_{1}\right)\right)$ are $\mathbb{Q}$-linearly independent over $\nu_{k_{1}}\left[k_{0} \backslash\{0\}\right]$ by the definition of $\Theta(x))$. But we may repeat this argument with $k_{1}$ in place of $k_{0}$ and, indeed, continue to do so to obtain, for each $l \in \mathbb{N}$, an elementary substructure, $k_{l}$ say, of $k$ such that $\operatorname{dim}\left(k_{l}\right)=\operatorname{dim}\left(k_{0}\right)+l$ and $\operatorname{valdim}\left(k_{l}\right) \geq \operatorname{valdim}\left(k_{0}\right)+2 l$. But this contradicts 10.4 when $l=\operatorname{dim}\left(k_{0}\right)+1$.

Before applying 10.4 to the situation of section 9, I require the following result on ordered $\mathbb{Q}$-vector spaces. 
10.5. Lemma. Let $V$ be an ordered $\mathbb{Q}$-vector space and $U$ a subspace of $V$ such that $V$ has dimension $n(\in \mathbb{N})$ over $U$. Then there exists a basis $0<v_{1}<v_{2}<\cdots<v_{n}$ for $V$ over $U$ such that if $v$ is any element of $V$ with $v>u$ for all $u \in U$ and if $v=\left(\sum_{i=1}^{n} q_{i} v_{i}+u_{0}\right)$ (where $\left.q_{1}, \ldots, q_{n} \in \mathbb{Q}, u_{0} \in U\right)$, then $|v|>q \cdot v_{j}$ for some positive $q \in \mathbb{Q}$ where $j=\max \left\{i: q_{i} \neq 0\right\}$.

Proof. Let $\bar{U}$ be the convex closure of $U$ in $V$. The result is trivial if $\bar{U}=V$. Otherwise, simply observe that there exists $l \in \mathbb{N}$, with $1 \leq l \leq n$, and Archmideanordered $\mathbb{Q}$-vector spaces $A_{1}, \ldots, A_{l}$ such that $V$ is isomorphic (as an ordered $\mathbb{Q}$ vector space) to $\bar{U} \times A_{1} \times \cdots \times A_{l}$ with reverse lexicographic ordering.

\section{BOUNDING THE SOLUTIONS TO EXPONENTIAL-POLYNOMIAL EQUATIONS AND THE COMPLETION OF THE PROOF OF THE SECOND MAIN THEOREM}

Recall from section 9 that $L_{\exp }$ and $T_{\exp }$ denote the language and theory of the structure $\langle\overline{\mathbb{R}} ; \exp \rangle$, respectively. I denote by $L_{e}$ and $T_{e}$ the language and theory of the structure $\langle\overline{\mathbb{R}} ; e ;\rangle$, where $e: \mathbb{R} \rightarrow \mathbb{R}: x \mapsto \exp \left(\left(1+x^{2}\right)^{-1}\right)$.

11.1. Theorem. The theory $T_{e}$ is smooth and model complete.

Proof. That $T_{e}$ is model complete follows from the first main theorem (see example (A) of section 1), and 0-minimality and condition (S1) follow from results in [13] (see also Corollary 3.5). For (S2), consider the function

$$
e^{*}: \mathbb{R} \rightarrow \mathbb{R}: x \mapsto \exp \left(x^{2} \cdot\left(1+x^{2}\right)^{-1}\right)
$$

and note that $e\left(x^{-1}\right)=e^{*}(x)$ for all $x \in \mathbb{R} \backslash\{0\}$. It follows that $e^{*}$ is definable in $\langle\mathbb{R} ; e\rangle$ without parameters. Notice also that $e$ and $e^{*}$ are both $C^{\infty}$ throughout $\mathbb{R}$. Now let $\phi\left(x_{1}, \ldots, x_{n}\right)$ be any formula of $L_{e}$. It easily follows from the model completeness of $T_{e}$ that there is a polynomial $\rho \in \mathbb{Z}\left[z_{1}, \ldots, z_{2 m+2 n}\right]$ (for some $m \in \mathbb{N}$ ) such that

$$
\begin{aligned}
&\langle\overline{\mathbb{R}} ; e\rangle \models \forall x_{1}, \ldots, x_{n}\left(\phi\left(x_{1}, \ldots, x_{n}\right) \leftrightarrow\right. \\
& \exists y_{1}, \ldots, y_{m} \rho\left(y_{1}, \ldots, y_{m}, e\left(y_{1}\right), \ldots, e\left(y_{m}\right),\right. \\
&\left.\left.x_{1}, \ldots, x_{n}, e\left(x_{1}\right), \ldots, e\left(x_{n}\right)\right)=0\right) .
\end{aligned}
$$

Condition (S2) now follows by considering, for each $s \subseteq\{1, \ldots, m\}$, the result of replacing $y_{j}$ by $y_{j}^{-1}$ and $e\left(y_{j}\right)$ by $e^{*}\left(y_{j}\right)$ (for each $j \in s$ ) in the function on the right hand side of $(*)$, and then multiplying by a suitably high power of $\prod_{j \in s} y_{j}$ to obtain a $C^{\infty}$ function (on $\mathbb{R}$ ), $F_{s}$ say. Thus, in the notation of (S2), $p$ is $2^{m}$, $N_{i}(\vec{y})$ is $\bigwedge_{j \in s_{i}} y_{j} \neq 0$ and $F_{i}$ is $F_{s_{i}}$, where $\left\{s_{i}: i<2^{m}\right\}$ is an enumeration of all the subsets of $\{1, \ldots, m\}$.

Suppose now that $k$ and $K$ are models of $T_{\exp }$ with $k \subseteq K$. Clearly, $k, K$ determine models of $T_{e}$ with the same underlying ordered field and I denote these ("restricted") models by $k^{\prime}, K^{\prime}$ respectively. Certainly $k^{\prime} \subseteq K^{\prime}$ so $k^{\prime} \preceq K^{\prime}$ by the model completeness of $T_{e}$ (Theorem 11.1).

Now suppose that $k^{*}$ is any model of $T_{e}$ such that $k^{\prime} \subseteq k^{*} \subseteq K^{\prime}$. Then for each $a \in k^{*}, \exp (a)$ is an element of (the domain of) $K$ which may or may not lie in $k^{*}$. Let $E\left(k^{*}\right)=\left\{a \in k^{*}: \exp (a) \in k^{*}\right\}$. Clearly $E\left(k^{*}\right)$ is a $\mathbb{Q}$-vector subspace of the additive group of $k^{*}$ (because $k^{*}$ is a real-closed ordered field and hence closed 
under taking rational powers of positive elements) and contains the additive group of $k$ as a subspace. It also contains $\operatorname{Fin}\left(k^{*}\right)$ as a subspace because if $a \in \operatorname{Fin}\left(k^{*}\right)$, then there exist $m \in \mathbb{Z}$ and $b \in k^{*}$ such that $\frac{m}{1+b^{2}}=a$, and then $\exp (a)=e(b)^{m}$, and $e(b)^{m} \in k^{*}$.

11.2. Lemma. In the above situation, suppose that $\operatorname{dim}_{k^{\prime}}\left(k^{*}\right)=n$ (as models of $\left.T_{e}\right)$, where $n \in \mathbb{N}$. Suppose further that $E\left(k^{*}\right)$ is at least $n$ dimensional over its subspace $k+F i n\left(k^{*}\right)$. Then for each $a \in E\left(k^{*}\right)$ there is some $b \in k$ such that $|a|<b$.

Proof. Suppose not. Let $U=k+\operatorname{Fin}\left(k^{*}\right)$ and choose some subspace $V$ of $E\left(k^{*}\right)$ such that $U \subseteq V, V$ is exactly $n$-dimensional over $U$, and such that $V$ contains an element $\alpha$ with $\alpha>b$ for all $b \in k$. Clearly this implies that $\alpha>b$ for all $b \in U$.

Let $0<v_{1}<\cdots<v_{n}$ be a basis for $V$ over $U$ as given by Lemma 10.5. Let $j$ be minimal such that $v_{j}>b$ for all $b \in U$.

Now consider the elements $\nu_{K}\left(\exp \left(v_{1}\right)\right), \ldots, \nu_{K}\left(\exp \left(v_{n}\right)\right)$ of the value group $V(K)$ of $K$. I claim that they are linearly independent over $\nu_{K}[k \backslash\{0\}]$. For if not there exist $q_{1}, \ldots, q_{n} \in \mathbb{Q}$, not all zero, and $c \in k \backslash\{0\}$ such that $c \exp \left(\sum_{i=1}^{n} q_{i} v_{i}\right) \in$ $\operatorname{Fin}(K) \backslash \mu(K)$ (using (v1) and (v3) of section 10). Clearly we may suppose that $c>0$, so $c=\exp (d)$ for some $d \in k$ (since $k \models T_{\exp }$ ). We thus have $\exp \left(d+\sum_{i=1}^{n} q_{i} v_{i}\right) \in \operatorname{Fin}(K) \backslash \mu(K)$ which, by standard properties of the exponential function, implies that $d+\sum_{i=1}^{n} q_{i} v_{i} \in \operatorname{Fin}(K)$, and hence $d+\sum_{i=1}^{n} q_{i} v_{i} \in$ $\operatorname{Fin}\left(k^{*}\right)$. But this contradicts the linear independence of $v_{1}, \ldots, v_{n}$ over $U$.

Now by Theorems 10.4 and 11.1 and the hypothesis that $\operatorname{dim}_{k^{\prime}}\left(k^{*}\right)=n$ it follows that valdim $k^{\prime}\left(k^{*}\right) \leq n$ and hence that $\nu_{K}\left(\exp \left(v_{1}\right)\right), \ldots, \nu_{K}\left(\exp \left(v_{n}\right)\right) \operatorname{span}$ $\nu_{K}\left[k^{*} \backslash\{0\}\right]$ over $\nu_{K}[k \backslash\{0\}]$ (note that $\left.\exp \left(v_{1}\right), \ldots, \exp \left(v_{n}\right) \in k^{*}\right)$. In particular

$$
\nu_{K}\left(v_{j}\right)=\nu_{K}(c)+\sum_{i=1}^{n} p_{i} \nu_{K}\left(\exp \left(v_{i}\right)\right)
$$

for some $c \in k \backslash\{0\}$ and $p_{1}, \ldots, p_{n} \in \mathbb{Q}$. Again, we may suppose that $c=\exp (d)$ for some $d \in k$ and hence, $\nu_{K}\left(v_{j}\right)=\nu_{K}\left(\exp \left(d+\sum_{i=1}^{n} p_{i} v_{i}\right)\right)$. By (v3) of section 10, this implies that $\frac{v_{j}}{N}<\exp \left(d+\sum_{i=1}^{n} p_{i} v_{i}\right)<N v_{j}$ for some $N \in \mathbb{N} \backslash\{0\}$. Now the left hand inequality here implies that $d+\sum_{i=1}^{n} p_{i} v_{i}>0$ (since certainly $\frac{v_{j}}{N}>1$ ). Further, if $p_{j}=p_{j+1}=\cdots=p_{n}=0$ we would have $0<d+\sum_{i=1}^{n} p_{i} v_{i}<b$ for some $b \in k$ and hence $\frac{v_{j}}{N}<\exp (b)$, which contradicts the choice of $v_{j}$ since $N \cdot \exp (b) \in k$. Thus $p_{i} \neq 0$ for some $i=j, \ldots, n$ and so, by the choice of $v_{i}, \ldots, v_{n}$, there exists $q \in \mathbb{Q}$ with $q>0$ such that $d+\sum_{i=1}^{n} p_{i} v_{i}>q v_{j}$ (see Lemma 10.5). But, by the right hand inequality above, this implies that $N v_{j}>\exp \left(q v_{j}\right)$. However, this is absurd since certainly $v_{j}>r$ for all $r \in \mathbb{N}$.

I now complete the proof of the main theorem. Recall from section 2 that we must consider the following situation:

We are given $n, m \in \mathbb{N}$ with $n \geq m>0, \vec{\alpha}=\left\langle\alpha_{1}, \ldots, \alpha_{n}\right\rangle \in K^{n}, l \in\{1, \ldots, n\}$, $s \subseteq\{1, \ldots, n\}$ with $|s|=m, l \in s$, and $f_{1}, \ldots, f_{n} \in M_{n}^{s}$ (cf. Definition 2.1) such that $f_{1}(\vec{\alpha})=\cdots=f_{n}(\vec{\alpha})=0$ and $J\left(f_{1}, \ldots, f_{n}\right)(\vec{\alpha}) \neq 0$. Further, $\left|\alpha_{l}\right|>b$ for all $b \in k$.

We must establish 9.3, for which it is clearly sufficient to show that $\alpha_{1}, \ldots, \alpha_{n}$ are $\mathbb{Q}$-linearly dependent over the subspace $k+F i n(K)$ of (the $\mathbb{Q}$-vector space) $K$. 
To do this, consider the submodel, $k^{*}$ say, of $K^{\prime}$ generated over $k^{\prime}$ by $\left\{\alpha_{i}: 1 \leq\right.$ $i \leq n\} \cup\left\{\exp \left(\alpha_{i}\right): i \in s\right\}$ using the Skolem functions of $T_{e}$. Then $k^{*} \models T_{e}$ and $k^{\prime} \subseteq k^{*} \subseteq K^{\prime}$. Obviously we have $\operatorname{dim}_{k^{\prime}}\left(k^{*}\right) \leq n+m$. I claim that, in fact, we have $\operatorname{dim}_{k^{\prime}}\left(k^{*}\right) \leq m$. Granted this claim, it follows from 11.2 that $E\left(k^{*}\right)$ has dimension at most $m-1$ over $k+F i n\left(k^{*}\right)$ (because $\alpha_{l} \in E\left(k^{*}\right)$ ). But $\left\{\alpha_{i}: i \in s\right\} \subseteq E\left(k^{*}\right)$ so $\left\{\alpha_{i}: i \in s\right\}$ is a $\mathbb{Q}$-linearly dependent set over $k+\operatorname{Fin}\left(k^{*}\right)$. A fortiori, $\alpha_{1}, \ldots, \alpha_{n}$ are $\mathbb{Q}$-linearly dependent over $k+F i n(K)$. To prove the claim let us suppose, for convenience, that $s=\{1, \ldots, m\}$. Set $\alpha_{n+i}=\exp \left(\alpha_{i}\right)$ for $i=1, \ldots, m$.

Now for $1 \leq i \leq n$, pick $g_{i} \in M_{n}^{\emptyset}\left[x_{n+1}, \ldots, x_{n+m}\right]$ such that $g_{i}\left(x_{1}, \ldots, x_{n}\right.$, $\left.\exp \left(x_{1}\right), \ldots, \exp \left(x_{m}\right)\right) \equiv f_{i}\left(x_{1}, \ldots, x_{m}\right)$, and set $g_{n+i}\left(x_{1}, \ldots, x_{n+m}\right)=\exp \left(x_{i}\right)-$ $x_{n+i}$ for $1 \leq i \leq m$. Then clearly $\left\langle\alpha_{1}, \ldots, \alpha_{n+m}\right\rangle$ is a solution to the system $g_{i}\left(x_{1}, \ldots, x_{n+m}\right)=0(1 \leq i \leq n+m)$. It is also easy to show, using the chain rule, elementary matrix algebra and the fact that $J\left(f_{1}, \ldots, f_{n}\right)\left(\alpha_{1}, \ldots, \alpha_{n}\right) \neq 0$, that $J\left(g_{1}, \ldots, g_{n+m}\right)\left(\alpha_{1}, \ldots, \alpha_{n+m}\right) \neq 0$. It follows that the row vectors $\left\langle\frac{\partial g_{1}}{\partial x_{i}}: 1 \leq\right.$ $i \leq n+m\rangle, \ldots,\left\langle\frac{\partial g_{n}}{\partial x_{i}}: 1 \leq i \leq n+m\right\rangle$ evaluated at $\left\langle\alpha_{1}, \ldots, \alpha_{n+m}\right\rangle$ are linearly independent over $K$ and hence that there exists a subset $u \subseteq\{1, \ldots, n+m\}$ of size $n$ such that the matrix

$$
\left(\frac{\partial g_{i}}{\partial x_{j}}\right)_{\substack{1 \leq i \leq n \\ j \in u}}
$$

is non-singular when evaluated at $\left\langle\alpha_{1}, \ldots, \alpha_{n+m}\right\rangle$. Now notice that $g_{1}, \ldots, g_{n}$ are $k^{\prime}$-definable functions (i.e. they are $L_{e}$-definable with parameters in $k$ ) so it clearly follows from Proposition 9.2(iii) (with $s=\emptyset$ ) that for each $j \in u, \alpha_{j}$ is $k^{\prime}$-definable from $\left\{\alpha_{i}: 1 \leq i \leq n+m, i \notin u\right\}$. Thus the submodel of $K^{\prime}$ generated over $k^{\prime}$ by $\left\{\alpha_{i}: 1 \leq i \leq n+m, i \notin u\right\}$ contains $\alpha_{1}, \ldots, \alpha_{n+m}$ and is therefore equal to $k^{*}$. Thus $\operatorname{dim}_{k^{\prime}}\left(k^{*}\right) \leq m$ as required.

\section{REFERENCES}

[1] J. Bridge, Begining model theory, Oxford Univ. Press, 1977. MR 58:27171

[2] B. I. Dahn, The limit behaviour of exponential terms, Fund. Math. 124 (1984), 169-186. MR 86f:03058

[3] J. Denef and L. van den Dries, P-adic and real subanalytic sets, Ann. of Math. 128 (1988), 79-138. MR 89k:03034

[4] J. Dieudonné, Foundations of modern analysis, Academic Press, San Diego, 1969. MR 50:1782

[5] A. Gabrielov, Projections of semi-analytic sets, Functional Anal. Appl. 2 (1968), 282-291. MR 39:7137

[6] A. G. Khovanskii, On a class of systems of transcendental equations, Soviet Math. Dokl. 22 (1980), 762-765. MR 82a:14006

[7] J. F. Knight, A. Pillay, and C. Steinhorn, Definable sets in ordered structures, II, Trans. Amer. Math. Soc. 295 (1986), 593-605. MR 88b:03050b

[8] S. Łojasiewicz, Ensembles semi-analytiques, mimeographed notes, IHES, 1965.

[9] L. Mirsky, Introduction to linear algebra, Oxford Univ. Press, 1955. MR 17:573a

[10] A. Pillay and C. Steinhorn, Definable sets in ordered structures. I Trans. Amer. Math. Soc. 295 (1986), 565-592. MR 88b:03050a

[11] A. Tarski, A decision method for elementary algebra and geometry, 2nd revised ed., Berkeley and Los Angeles, 1951. MR 13:423a

[12] L. van den Dries, Remarks on Tarski's problem concerning $\langle R,+, \cdot, \exp \rangle$, Logic Colloquium 1982, North Holland, 1984, p. 97-121. MR 86b:03052

[13] _ A generalization of the Tarski-Seidenberg theorem, and some nondefinability results, Bull. Amer. Math. Soc. (N.S.) 15 (1986), 189-193. MR 88b:03048 
$[14] \ldots$, On the elementary theory of restricted elementary functions, J. Symbolic Logic 53 (1988), 796-808. MR 89i:03074

[15] _ Tame topology and 0-minimal structures, mimeographed notes, University of Illinois at Urbana-Champaign, 1991.

[16] A. J. Wilkie, On the theory of the real exponential field, Illinois J. Math. 33 (1989), 384-408. MR 90i:03042

[17] H. Wolter, On the model theory of exponential fields (survey), Logic Colloquium 1984, NorthHolland, 1986, pp. 343-353. MR 88a:03082

Mathematical Institute, 24-29 St. Giles, Oxford OX1 3LB, United Kingdom

E-mail address: wilkie@maths.oxford.ac.uk 